\title{
GEOMETRIC RIGIDITY OF SPHERICAL HYPERSURFACES IN QUATERNIONIC MANIFOLDS*
}

\author{
YOSHINOBU KAMISHIMA ${ }^{\dagger}$
}

\begin{abstract}
This article concerns a geometric structure on $(4 n+3)$-dimensional smooth manifolds. The isometry group of quaternionic hyperbolic space acts transitively on the boundary sphere as projective transformations. It gives a geometry $\left(\operatorname{PSp}(n+1,1), S^{4 n+3}\right)$. A $(4 n+3)$-manifold locally modelled on this geometry is said to be a spherical pseudo-quaternionic manifold. We discuss a Carnot-Carathéodory structure on spherical pseudo-quaternionic manifolds in connection with the Sasakian 3-structure. Using superrigidity in quaternionic hyperbolic group, we shall prove the geometric rigidity of compact spherical pseudo-quaternionic $(4 n+3)$-manifolds when the fundamental group is isomorphic to either an amenable group or a quaternionic hyperbolic group.
\end{abstract}

Introduction. A spherical pseudo-quaternionic structure is a geometric structure on a $(4 n+3)$-manifold locally modelled on the sphere $S^{4 n+3}$ with coordinate changes lying in the Lorentz group $\operatorname{PSp}(n+1,1)$. Here $\operatorname{PSp}(n+1,1)$ is isomorphic to the isometry group Iso $\left(\mathbb{H}_{\mathbb{F}}^{n+1}\right)$ of the quaternionic hyperbolic space $\mathbb{H}_{\mathbb{F}}^{n+1}$ where $\mathbb{F}$ stands for the noncommutative field of quaternions. The space $\mathbb{H}_{F}^{n+1}$ has the projective compactification whose boundary is the sphere $S^{4 n+3}$ on which $\operatorname{PSp}(n+1,1)$ acts as projective transformations. The pair $\left(\operatorname{PSp}(n+1,1), S^{4 n+3}\right)$ is said to be spherical pseudo-quaternionic geometry (cf. [19], [6]). A $(4 n+3)$-manifold locally modelled on this geometry is said to be a spherical pseudo-quaternionic manifold. In this paper we shall study geometric rigidity of compact spherical pseudo-quaternionic manifolds. A typical example of spherical pseudo-quaternionic manifolds is a homogeneous space and its compact quotient $M$. That is, there is a transitive group of spherical pseudoquaternionic transformations of the universal covering space $\tilde{M}$ which has a discrete subgroup $\Gamma$ acting properly discontinuously on $\tilde{M}$ with compact quotient $\tilde{M} / \Gamma$. For our geometric use, we follow the construction of hyperboloid in real hyperbolic geometry. Let $S^{4 n+3}-S^{4 n-1}$ be the sphere complement where $S^{4 n-1}$ is the boundary of the quaternionic hyperbolic space $\mathbb{H}_{\mathbb{F}}^{n}$. It turns out that $S^{4 n+3}-S^{4 n-1}$ is the total space of the principal bundle: $\operatorname{Sp}(1) \rightarrow S^{4 n+3}-S^{4 n-1} \longrightarrow \mathbb{H}_{\mathbb{F}}^{n}$ and the subgroup of $\operatorname{PSp}(n+1,1)$ preserving $S^{4 n-1}$ is isomorphic to the group $\operatorname{Sp}(n, 1) \cdot \operatorname{Sp}(1)$. Moreover, the fundamental result in semi-Riemannian geometry is that $S^{4 n+3}-S^{4 n-1}$ is a simply connected geodesically complete semi-Riemannian manifold of type $(3,4 n)$ with constant curvature -1 (cf. [29], [46]). If $\pi$ is a torsionfree discrete uniform subgroup of $\operatorname{Sp}(n, 1) \cdot \operatorname{Sp}(1)$, then the orbit space $M_{0}=S^{4 n+3}-S^{4 n-1} / \pi$ is a compact spherical pseudo-quaternionic manifold which has the fibration over the compact quaternionic hyperbolic space form:

$$
\mathrm{Sp}(1) \rightarrow M_{0} \stackrel{\nu}{\longrightarrow} \mathbb{H}_{\mathrm{F}}^{n} / \Gamma
$$

Here $\pi$ is mapped isomorphically onto a torsionfree discrete uniform subgroup $\Gamma \subset$ $\operatorname{PSp}(n, 1)=\operatorname{Iso}\left(\mathbb{H}_{\mathbb{F}}^{n}\right)$. More generally, we have the homogeneous spherical pseudoquaternionic spaces and their compact quotients:

$$
\mathrm{Sp}(m) \times \Delta \mathrm{Sp}(1) \times \mathrm{Sp}(n-m) \backslash \mathrm{Sp}(m, 1) \cdot \mathrm{Sp}(n-m+1) / \pi=S^{4 n+3}-S^{4 m-1} / \pi,
$$

${ }^{*}$ Received February 16, 1998; accepted for publication January 13, 1999.

†Department of Mathematics, Tokyo Metropoiltan University, Minami-Ohsawa 1-1, Hachioji, Tokyo 192-0397, JAPAN (kami@comp.metro-u.ac.jp). 
where $1 \leqq m \leqq n$ and the fundamental group $\pi$ is isomorphic to a discrete uniform subgroup of $\operatorname{PSp}(m, 1)$. (Compare $\S 1$.) By using the Margulis' superrigidity by Corlette [11], we prove that the converse is also true.

THEOREM A. Let $M$ be a compact spherical pseudo-quaternionic $(4 n+3)$-manifold whose fundamental group $\pi_{1}(M)$ is isomorphic to a discrete uniform subgroup of $\operatorname{PSp}(m, 1)$ for some $m$ where $2 \leqq m \leqq n$. Then $M$ is pseudo-quaternionically isomorphic to the double coset space $\operatorname{Sp}(m) \times \Delta \mathrm{Sp}(1) \times \operatorname{Sp}(n-m) \backslash \mathrm{Sp}(m, 1) \cdot \operatorname{Sp}(n-m+$ 1) $/ \Gamma$ where $m=2, \cdots, n$.

Let $\mathcal{T}\left(M_{0}\right)$ be the deformation space of spherical pseudo-quaternionic structures on marked manifolds homeomorphic to $M_{0}$. There is the natural map hol : $\mathcal{T}\left(M_{0}\right) \rightarrow \operatorname{Hom}(\pi, \operatorname{PSp}(n+1,1)) / \operatorname{PSp}(n+1,1)$ which assigns to a marked structure its holonomy representation. As an application, we have the following. (Compare [14].)

CoROllary B. The map hol maps $\mathcal{T}\left(M_{0}\right)$ homeomorphically onto a connected component in $\operatorname{Hom}(\pi, \operatorname{PSp}(n+1,1)) / \mathrm{PSp}(n+1,1)$. Moreover, the connected component consists of the set of Fuchsian representations which is diffeomorphic to $\operatorname{Hom}(\pi, \operatorname{Sp}(1)) / \operatorname{Sp}(1)$.

In order to prove Theorem A and Corollary B, we examine the holonomy representation $\rho: \pi_{1}(M) \rightarrow \operatorname{PSp}(n+1,1)$. As a spherical pseudo-quaternionic structure, the sphere with one point removed, $S^{4 n+3}-\{\infty\}$, is identified with the Heisenberg nilpotent Lie group $\mathcal{M}$. Here $\mathcal{M}$ lies in the central extension $1 \rightarrow \mathbb{R}^{3} \rightarrow \mathcal{M} \stackrel{\nu}{\longrightarrow} \mathbb{F}^{n} \rightarrow 1$ for which $\mathbb{F}^{n}$ is the $n$-dimensional quaternionic vector space (cf. $\left.\S 1\right)$. Let $\operatorname{Sim}(\mathcal{M})$ be the subgroup of $\operatorname{PSp}(n+1,1)$ whose elements leave $\mathcal{M}$ invariant (equivalently, each element stabilizes the point at infinity $\{\infty\})$. Recall that an amenable closed subgroup of $\operatorname{PSp}(n+1,1)$ is conjugate to a subgroup of either $\operatorname{Sim}(\mathcal{M})$ or a maximal compact subgroup $\operatorname{Sp}(n+1) \cdot \operatorname{Sp}(1)$. Given a group $G$, a representation $\rho: G \rightarrow \operatorname{PSp}(n+1,1)$ is said to be amenable if the closure of the image $\overline{\rho(G)}$ is amenable. To apply the superrigidity, it is necessary for a representation to be Zariski-dense. First of all we must avoid the case of amenable holonomy representations. We have the following classification.

THEOREM C. Let $M$ be a compact spherical pseudo-quaternionic $(4 n+3)$-manifold. If the holonomy group is amenable, then $M$ is finiteily covered by the sphere $S^{4 n+3}$, a Hopf manifold $S^{1} \times S^{4 n+2}$ or a nilmanifold $\mathcal{M} / \Gamma$.

Our method to the proofs is to study a Carnot-Carathéodory structure on a spherical pseudo-quaternionic manifold from the viewpoint of gauge theory, which is based on the H. Weyl's conformal structure. A quaternionic Carnot-Carathéodory structure on a $(4 n+3)$-manifold $M$ consists of a nondegenerate codimension 3-subbundle $B$ of $T M$ each fiber of which is endowed with an $n$-dimensional quaternionic vector space (cf. [32],[34]). Our notion of quaternionic Carnot-Carathéodory structure generalizes the Sasakian 3-structure. Classically the Sasakian 3-structure [40],[41] is known to be a geometric structure on $(4 n+3)$-manifolds as an analogue of quaternionic Kähler manifolds such as the quaternionic projective space $\mathbb{F P}^{n}$ (cf. [16],[28],[38]). In particular the canonical Sasakian 3 -structure on $S^{4 n+3}$ induces the Hopf bundle: $\operatorname{Sp}(1) \rightarrow S^{4 n+3} \stackrel{\pi}{\longrightarrow} \mathbb{F P}^{n}$. The connection form defines an $\mathfrak{s p}(1)$-valued one-form $\theta$ on $S^{4 n+3}$ for which Null $\theta=\left\{X \in T S^{4 n+3} \mid \theta(X)=0\right\}$ gives rise to a nondegenerate codimension 3 -subbundle. We see that $\left(S^{4 n+3}\right.$, Null $\left.\theta\right)$ is a quaternionic Carnot-Carathéodory manifold. Then it is a fundamental purpose to exhibit spherical pseudo-quaternionic geometry on $S^{4 n+3}$ as an analogue of spherical Cauchy-Riemann geometry [6], or that of conformally flat geometry. 
Proposition D. If Aut QCC $_{\mathrm{QCC}}\left(S^{4 n+3}\right)$ is the group of all quaternionic CarnotCarathéodory transformations of $\left(S^{4 n+3}\right.$, Null $\left.\theta\right)$, then $\operatorname{Aut}_{Q \mathrm{CC}}\left(S^{4 n+3}\right)=\operatorname{PSp}(n+1,1)$. That is, the spherical pseudo-quaternionic geometry $\left(\mathrm{PSp}(n+1,1), S^{4 n+3}\right)$ coincides with the quaternionic Carnot-Carathéodory geometry on $S^{4 n+3}$.

By this proposition, a spherical pseudo-quaternionic $(4 n+3)$-manifold $M$ will be a quaternionic Carnot-Carathéodory manifold. For example, the quaternionic Carnot-Carathéodory structure to the domain $S^{4 n+3}-S^{4 n-1}$ is mapped isometrically at each point onto the quaternionic hyperbolic geometry $\left(\operatorname{PSp}(n, 1), \mathbb{H}_{\mathbb{F}}^{n}\right)$ with the automorphism group $\operatorname{Aut}_{\mathrm{QCC}}\left(S^{4 n+3}-S^{4 n-1}\right)=\operatorname{Sp}(n, 1) \cdot \operatorname{Sp}(1)$. The quaternionic Carnot-Carathéodory metric on $\mathcal{M}$ plays the same role as the euclidean metric on $\mathbb{F}^{n}$. Concerned with the proof of Theorem $\mathrm{C}$, a compact spherical pseudo-quaternionic $(4 n+3)$-manifold with amenable holonomy provides a holonomy-invariant closed subset outside the developing image in $S^{4 n+3}$ by using the idea of Fried [12]. Then we shall prove Theorem $\mathrm{C}$ by showing that the developing map is a covering map onto its image.

Finally we remark the non-rigidity. There are examples of non-homogeneous spherical pseudo-quaternionic manifolds and compact quotients. Given such a compact manifold $M_{1}=S^{4 n+3}-S^{n} / \Gamma$ (cf. Corollary 6 , Note 7 ), there is a nontrivial family of developing pairs $\left(\operatorname{dev}_{t}, \rho_{t}\right)(t \in \mathrm{Sp}(1))$ of spherical pseudo-quaternionic structures on $M_{1}$. This is obtained by the bending deformation.

\section{Spherical pseudo-quaternionic geometry.}

1.1. Spherical pseudo-quaternionic structure. Let $\mathbb{F}^{n+2}$ denote the quaternionic vector space, equipped with the Hermitian form

$$
\mathcal{B}(z, w)=-\bar{z}_{1} w_{1}+\bar{z}_{2} w_{2}+\cdots+\bar{z}_{n+2} w_{n+2} .
$$

Consider the following subspaces in $\mathbb{F}^{n+2}-\{0\}$ :

$$
\begin{aligned}
& V_{0}^{4 n+7}=\left\{z \in \mathbb{F}^{n+2} \mid \mathcal{B}(z, z)=0\right\}, \\
& V_{-}^{4 n+8}=\left\{z \in \mathbb{F}^{n+2} \mid \mathcal{B}(z, z)<0\right\} .
\end{aligned}
$$

Let $P: \mathbb{F}^{n+2}-\{0\} \longrightarrow \mathbb{F P}^{n+1}$ be the canonical projection onto the quaternionic projective space. By definition [8], the quaternionic hyperbolic space $\mathbb{H}_{\mathbb{F}}^{n+1}$ is defined to be $P\left(V_{-}^{4 n+8}\right)$. Let $\mathrm{GL}(n+2, \mathbb{F})$ be the group of all invertible $(n+2) \times(n+2)$-matrices with quaternion entries. The group $\operatorname{Sp}(n+1,1)$ is the subgroup of $\mathrm{GL}(n+2, \mathbb{F})$ whose elements preserve the form $\mathcal{B}$. The action of $\operatorname{Sp}(n+1,1)$ on $V_{-}^{4 n+8}$ induces an action on $\mathbb{H}_{\mathbb{F}}^{n+1}$. The kernel of this action is the center $\mathbb{Z} / 2=\{ \pm 1\}$ and the quaternionic hyperbolic group $\operatorname{PSp}(n+1,1)$ is defined to be the quotient of $\operatorname{Sp}(n+1,1)$ by the center. It is known that $\mathbb{H}_{\mathbb{F}}^{n+1}$ is a complete simply connected Riemannian manifold of $-1 \leqq$ sectional curvature $\leqq-\frac{1}{4}$, and with the group of isometries $\operatorname{PSp}(n+1,1)$ (cf. [25]).

The projective compactification of $\mathbb{H}_{\mathbb{F}}^{n+1}$ is obtained by taking the closure $\overline{\mathbb{H}}_{\mathbb{F}}^{n+1}$ of $\mathbb{H}_{\mathbb{F}}^{n+1}$ in $\mathbb{F P P}^{n+1}$. Then it follows that $\overline{\mathbb{H}}_{\mathbb{F}}^{n+1}=\mathbb{H}_{\mathbb{F}}^{n+1} \cup P\left(V_{0}^{4 n+7}\right)$. The boundary $P\left(V_{0}^{4 n+7}\right)$ of $\mathbb{H}_{\mathbb{F}}^{n+1}$ is the standard sphere of dimension $4 n+3$. Put $P\left(V_{0}^{4 n+7}\right)=S^{4 n+3}$. Then the hyperbolic action of $\operatorname{PSp}(n+1,1)$ on $\mathbb{H}_{F}^{n+1}$ extends to a smooth action on $S^{4 n+3}$ acting as projective transformations because the compactification $\mathbb{H}_{\mathbb{F}}^{n+1} \cup S^{4 n+3}$ sits inside $\mathbb{F P}^{n+1}$. The action of $\operatorname{PSp}(n+1,1)$ is transitive on $S^{4 n+3}$ whose stabilizer at infinity $\{\infty\}$ is isomorphic to $\operatorname{Sim}(\mathcal{M})$. (Compare 1.2.) We then call the pair $\left(\operatorname{PSp}(n+1,1), S^{4 n+3}\right)$ spherical pseudo-quaternionic geometry. Notice that the same 
construction for the real (resp. complex) hyperbolic space is referred to conformally flat geometry $\left(\mathrm{PO}(n, 1), S^{n}\right)$, spherical $C R$-geometry $\left(\mathrm{PU}(n+1,1), S^{2 n+1}\right)$ (cf. [13], $[6])$.

Let $M$ be a smooth manifold of dimension $4 n+3$. Suppose that $\left\{U_{\alpha}, \phi_{\alpha}\right\}_{\alpha \in \Lambda}$ is a maximal collection of charts of $M$ satisfying that $M=\bigcup_{\alpha \in \Lambda} U_{\alpha}, \quad \phi_{\alpha}: U_{\alpha} \rightarrow \phi_{\alpha}\left(U_{\alpha}\right)$ ( $\subset$ $S^{4 n+3}$ ) is a homeomorphism, and if $U_{\alpha} \cap U_{\beta} \neq \emptyset$, then the coordinate change $g_{\alpha \beta}=$ $\phi_{\beta} \circ \phi_{\alpha}^{-1}$ extends to an element of $\operatorname{PSp}(n+1,1)$.

Such a collection of charts is said to give a uniformization on $M$. An equivalence class of uniformizations (by refinement) is called a spherical pseudo-quaternionic structure on $M$. A manifold equipped with this structure is said to be a spherical pseudo-quaternionic manifold $M$.

Denote by $\operatorname{Aut}_{\mathrm{PQ}}(M)$ the group of spherical pseudo-quaternionic transformations of $M$. Using a uniformization on $M$ (cf. [28]), there is a developing pair: ( $\rho$, dev) : $\left(\operatorname{Aut}_{\mathrm{PQ}}(\tilde{M}), \tilde{M}\right) \longrightarrow\left(\mathrm{PSp}(n+1,1), S^{4 n+3}\right)$ where $\tilde{M}$ is the universal covering space of $M$ and $\pi_{1}(M) \subset \operatorname{AutPQ}_{\mathrm{P}}(\tilde{M})$.

1.2. Quaternionic Heisenberg geometry. Let $P:\left(\operatorname{Sp}(n+1,1), V_{-}^{4 n+8} \cup\right.$ $\left.V_{0}^{4 n+7}\right) \longrightarrow\left(\operatorname{PSp}(n+1,1), \mathbb{H}_{\mathrm{F}}^{n+1} \cup S^{4 n+3}\right)$ be the equivariant projection. If $\{\infty\}$ is the point at infinity of $S^{4 n+3}$, then the stabilizer $\operatorname{PSp}(n+1,1)_{\infty}$ is a noncompact maximal amenable Lie subgroup of $\operatorname{PSp}(n+1,1)$. Let $\left\{e_{1}, \ldots, e_{n+2}\right\}$ be the standard basis of $\mathbb{F}^{n+2}$ with respect to the Hermitian form $\mathcal{B}$, i.e., $\mathcal{B}\left(e_{1}, e_{1}\right)=-1, \mathcal{B}\left(e_{i}, e_{j}\right)=$ $\delta_{i j}(i, j=2, \ldots, n+2), \mathcal{B}\left(e_{1}, e_{j}\right)=0(j=2, \ldots, n+2)$. Since $V_{0}^{4 n+7}$ is a cone, we can assume that the inverse image $P^{-1}(\infty)$ consists of a quaternionic line passing through the vector $f_{1}=\left(e_{1}+e_{n+2}\right) / \sqrt{2}$ (that is, $P\left(f_{1}\right)=\infty$ ). If $H$ is a subgroup of $\operatorname{Sp}(n+1,1)$ which leaves $f_{1}$ invariant, then $P H$ is isomorphic to $\operatorname{PSp}(n+1,1)_{\infty}$. Put $f_{n+2}=\left(e_{1}-e_{n+2}\right) / \sqrt{2}$. Now each element $g$ of $H$ has the following form with respect to the basis $\left\{f_{1}, e_{2}, \ldots, e_{n+1}, f_{n+2}\right\}$ :

$$
g=\left(\begin{array}{ccc}
\lambda & x & z \\
0 & \mathrm{~B} & y \\
0 & 0 & \mu
\end{array}\right)
$$

where $\lambda, \mu \in \mathbb{F}^{*}, B$ is an $(n, n)$-matrix, $x$ is an $n$-th line vector, and $y$ is an $n$ th column vector. As $\mathcal{B}(g z, g w)=\mathcal{B}(z, w)$ for arbitrary $z, w \in \mathbb{F}^{n+2}$, we have the following relations (cf. [8]).

$$
\begin{aligned}
& \text { (1) } \bar{\lambda} \mu=1, \quad x=\lambda^{t} \bar{y} B . \\
& \text { (2) } \bar{z} \mu+\bar{\mu} z=|y|^{2}, \quad B \in \operatorname{Sp}(n) .
\end{aligned}
$$

Let $\mathcal{M}$ be the subgroup consisting of the following matrices;

$$
\left(\begin{array}{lll}
1 & x & z \\
0 & \mathrm{I} & y \\
0 & 0 & 1
\end{array}\right)
$$

satisfying that $\operatorname{Re} z=\frac{|y|^{2}}{2}, x={ }^{t} \bar{y}$. (Note that this follows from (1), (2).) Putting $z=\frac{|y|^{2}}{2}+i \alpha+j \beta+k \gamma$, there is a one-to-one correspondence between the product 
$\mathbb{R}^{3} \times \mathbb{F}^{n}$ and $\mathcal{M}$ :

$$
((\alpha, \beta, \gamma), y)=\left(\begin{array}{ccc}
1 & { }^{t} \bar{y} & \frac{|y|^{2}}{2}+i \alpha+j \beta+k \gamma \\
0 & \mathrm{I} & y \\
0 & 0 & 1
\end{array}\right)
$$

Then the one-to-one correspondence gives a group law on the product $\mathbb{R}^{3} \times \mathbb{F}^{n}$. As a consequence, $\mathcal{M}$ is the product $\mathbb{R}^{3} \times \mathbb{F}^{n}$ with group law:

$$
(a, y) \cdot(b, z)=(a+b+\operatorname{Im}\langle y, z>, y+z) .
$$

Here $<>$ is the Hermitian inner product and $\operatorname{Im}<>$ is the imaginary part. $\mathcal{M}$ is nilpotent because $[\mathcal{M}, \mathcal{M}]=\mathbb{R}^{3}$ which is the center consisting of the form $((\alpha, \beta, \gamma), 0)$. $\mathcal{M}$ is called the Heisenberg nilpotent Lie group. (Compare [13].) Moreover $H$ is isomorphic to the semidirect product $\mathcal{M} \rtimes\left(\operatorname{Sp}(n) \times \mathbb{F}^{*}\right)$. We define the subgroup $\operatorname{Sim}(\mathcal{M})$ of $\operatorname{PSp}(n+1,1)$ to be $P H=\operatorname{PSp}(n+1,1)_{\infty}$. Then $\operatorname{Sim}(\mathcal{M})$ is isomorphic to the semidirect product $\mathcal{M} \rtimes\left(\operatorname{Sp}(n) \cdot \operatorname{Sp}(1) \times \mathbb{R}^{+}\right)$. The action of $\operatorname{Sp}(n) \cdot \operatorname{Sp}(1) \times \mathbb{R}^{+}$ on $\mathcal{M}$ is given as follows: if $(A \cdot g, t) \in \mathrm{Sp}(n) \cdot \mathrm{Sp}(1) \times \mathbb{R}^{+}$and $(a, y) \in \mathcal{M}$, then

$$
(A \cdot g, t) \circ(a, y)=\left(t^{2} \cdot g a g^{-1}, t \cdot A y g^{-1}\right) .
$$

Choosing $x_{0}=P\left(f_{n+2}\right) \in S^{4 n+3}-\{\infty\}, \mathcal{M}$ acts simply transitively on $S^{4 n+3}-\{\infty\}$ by $\rho(g)=g x_{0}$ for $g \in \mathcal{M}$. $S^{4 n+3}-\{\infty\}$ is identified with $\mathcal{M}$ as a spherical pseudoquaternionic structure. The pair $(\operatorname{Sim}(\mathcal{M}), \mathcal{M})$ is called quaternionic Heisenberg geometry.

Choosing a torsionfree discrete cocompact subgroup $\Gamma$ from $\mathcal{M} \rtimes(\operatorname{Sp}(n) \cdot \operatorname{Sp}(1))$, we have a fibration of an infranilmanifold as a compact spherical pseudo-quaternionic manifold;

$$
T^{3} \rightarrow \mathcal{M} / \Gamma \longrightarrow \mathbb{F}^{n} / \hat{\Gamma}
$$

where $T^{3}$ is the 3 -torus and $\mathbb{F}^{n} / \hat{\Gamma}\left(\hat{\Gamma} \subset \mathrm{E}(n)=\mathbb{F}^{n} \rtimes(\operatorname{Sp}(n) \cdot \operatorname{Sp}(1))\right.$ is the quaternionic euclidean flat orbifold.

Let $\mathcal{M}-\{0\}\left(=S^{4 n+3}-\{0, \infty\}\right) \approx \mathbb{R}^{+} \times S^{4 n+2}$. Then it follows that (cf. [19])

$$
\operatorname{Aut}_{\mathrm{PQ}}\left(\mathbb{R}^{+} \times S^{4 n+2}\right)=\left(\mathrm{O}(1,1)^{0} \rtimes \mathbb{Z} / 2\right) \times(\mathrm{O}(3) \times \mathrm{Sp}(n) \cdot \mathrm{Sp}(1)) .
$$

Choosing a torsion free discrete cocompact subgroup $\Delta$ of $\operatorname{Sp}(n) \cdot \operatorname{Sp}(1) \times \mathbb{R}^{+}$we obtain an infra-Hopf manifold

$$
\mathbb{R}^{+} \times S^{4 n+2} / \Delta \approx S^{1} \times S^{4 n+2} / G,
$$

where $G$ is a finite subgroup of $\operatorname{Aut}_{\mathrm{PQ}}\left(S^{1} \times S^{4 n+2}\right)=\left(S^{1} \rtimes \mathbb{Z} / 2\right) \times(\mathrm{O}(3) \times \operatorname{Sp}(n) \cdot \operatorname{Sp}(1))$. In particular, the Hopf manifold $S^{1} \times S^{4 n+2}$ is a spherical pseudo-quaternionic manifold. Similar to the conformal, $C R$-cases, there is an orientation reversing involution $\tau$ in $\operatorname{Aut}_{\mathrm{PQ}}\left(S^{4 n+3}-\{0, \infty\}\right)=\operatorname{Aut}_{\mathrm{PQ}}\left(\mathbb{R}^{+} \times S^{4 n+2}\right)$, we can perform an operation of the connected sum which is closed under the spherical pseudo-quaternionic structure. (Compare [6], [28].) We obtain that

Proposition 1. Let $M_{1}, M_{2}$ be spherical pseudo-quaternionic manifolds. Then the connected sum $M_{1} \# M_{2}$ supports a spherical pseudo-quaternionic structure. 
1.3. Homogeneous spherical pseudo-quaternionic manifolds. We introduce another quadric $V_{-1}^{4 n+3}=\left\{z \in \mathbb{F}^{n+1}-\{0\} \mid \mathcal{B}(z, z)=-1\right\}$. In the quaternion case, the group $\mathrm{GL}(n+1, \mathbb{F})$ is acting on $\mathbb{F}^{n+1}$ from the left and $\mathbb{F}^{*}=\mathrm{GL}(1, \mathbb{F})$ acting as the scalar multiplications from the right. We have the following equivariant principal bundles over $\mathbb{F P}^{n}$.

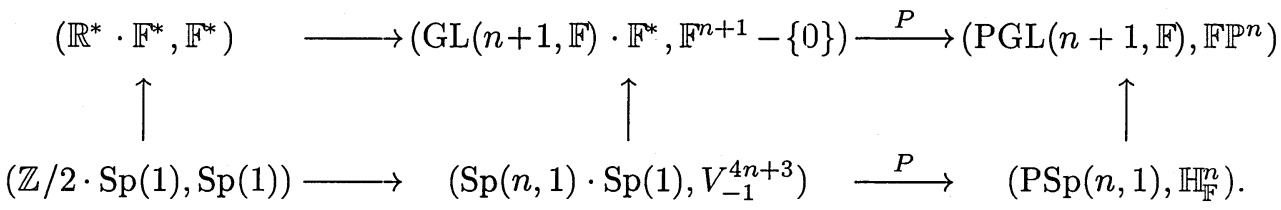

Then $V_{-1}^{4 n+3}$ is known to be a simply connected geodesically complete semi-Riemannian manifold of type $(3,4 n)$ with constant curvature -1 (cf. [29]). Note that the group of isometries of $V_{-1}^{4 n+3}$ is $\operatorname{Sp}(n, 1) \cdot \mathrm{Sp}(1)$. Let $\pi$ be a torsionfree discrete uniform subgroup of $\mathrm{Sp}(n, 1) \cdot \mathrm{Sp}(1)$. From the above sequence, $P$ maps isomorphically onto a torsionfree discrete uniform subgroup $\Gamma$ of $\operatorname{PSp}(n, 1)$. Since $\operatorname{Sp}(1)$ is compact, $\pi$ acts properly discontinuously and freely on $V_{-1}^{4 n+3}$. Put $M_{0}=V_{-1}^{4 n+3} / \pi$. By the above diagram, there is a fiber bundle over the compact quaternionic hyperbolic manifold

$$
\mathrm{Sp}(1) \rightarrow V_{-1}^{4 n+3} / \pi \longrightarrow \mathbb{H}_{\mathrm{F}}^{n} / \Gamma
$$

We give examples of Riemannian homogeneous spaces as spherical pseudo-quaternionic structure and their compact quotients. Let $S^{4 n+3}-S^{4 m-1}$ be the sphere complement $(1 \leqq m \leqq n)$, which is isomorphic to the quotient space $P\left(V_{-1}^{4 m+3} \times\right.$ $\left.S^{4(n-m)+3}\right)$ by chasing the equivariant principal bundle:

$$
\begin{aligned}
& (\mathbb{Z} / 2, \operatorname{Sp}(1)) \\
& \left(G, V_{-1}^{4 m+3} \times S^{4(n-m)+3}\right) \\
& \downarrow P \\
& \left(P G, P\left(V_{-1}^{4 m+3} \times S^{4(n-m)+3}\right)\right)=S^{4 n+3}-S^{4 m-1} \subset \mathbb{F P}^{n+1} \text {, }
\end{aligned}
$$

where $G=\operatorname{Sp}(m, 1) \times \operatorname{Sp}(n-m+1) \stackrel{P}{\longrightarrow} \operatorname{Sp}(m, 1) \cdot \operatorname{Sp}(n-m+1)$. If $\operatorname{Aut}_{\mathrm{PQ}}\left(S^{4 n+3}-\right.$ $\left.S^{4 m-1}\right)$ is the subgroup of $\operatorname{PSp}(n+1,1)$ preserving $S^{4 m-1}$, then it is isomorphic to $P G=\operatorname{Sp}(m, 1) \cdot \operatorname{Sp}(n-m+1)$. Let

$$
\Delta \mathrm{Sp}(1)=\left\{\left(\begin{array}{rr}
\lambda & 0 \\
0 & \mathrm{I}_{m}
\end{array}\right),\left(\begin{array}{rr}
\lambda & 0 \\
0 & \mathrm{I}_{n-m}
\end{array}\right)\right\} /\{ \pm 1\} \subset \mathrm{Sp}(m, 1) \cdot \operatorname{Sp}(n-m+1) .
$$

Then $\operatorname{Sp}(m) \times \Delta \operatorname{Sp}(1) \times \operatorname{Sp}(n-m) \backslash \operatorname{Sp}(m, 1) \cdot \operatorname{Sp}(n-m+1)=S^{4 n+3}-S^{4 m-1}$, which is a Riemannian homogeneous space because the stabilizer $\operatorname{Sp}(m) \times \Delta \operatorname{Sp}(1) \times \operatorname{Sp}(n-m)$ is compact. Choosing a torsionfree discrete uniform subgroup $\pi \subset \operatorname{Sp}(m, 1) \cdot \operatorname{Sp}(n-$ $m+1)$, we obtain a double coset space $S^{4 n+3}-S^{4 m-1} / \pi$ as a compact spherical pseudo-quaternionic manifold. As $\pi$ is mapped isomorphically onto a discrete uniform subgroup $\Gamma \subset \operatorname{PSp}(m, 1)$, there is a fiber bundle over the quaternionic hyperbolic manifold $\mathbb{H}_{\mathbb{F}}^{m} / \Gamma$.

$$
S^{4(n-m)+3} \rightarrow S^{4 n+3}-S^{4 m-1} / \pi \rightarrow \longrightarrow \mathbb{H}_{\mathbb{F}}^{m} / \Gamma .
$$


In particular when $m=n, S^{4 n+3}-S^{4 n-1}=P\left(V_{-1}^{4 n+3} \times S^{3}\right)=V_{-1}^{4 n+3}$.

PROPOSITION 2. $M_{0}$ is a compact spherical pseudo-quaternionic manifold whose developing image is a homogeneous space $S^{4 n+3}-S^{4 n-1}$.

1.4. Proper action of subgroups of $\operatorname{PSp}(n+1,1)$. For our later use, we prove the existence of proper actions of connected Lie groups on the sphere complement which is not homogeneous. Let $(\operatorname{Sim}(\mathcal{M}), \mathcal{M})$ be the Heisenberg geometry for which $\operatorname{Sim}(\mathcal{M})=\mathcal{M} \rtimes\left(\operatorname{Sp}(n) \cdot \operatorname{Sp}(1) \times \mathbb{R}^{+}\right)$. There is the equivariant principal bundle:

$$
\mathbb{R}^{3} \rightarrow(\operatorname{Sim}(\mathcal{M}), \mathcal{M}) \stackrel{\nu}{\longrightarrow}\left(\operatorname{Sim}\left(\mathbb{F}^{n}\right), \mathbb{F}^{n}\right) .
$$

The subgroup $\operatorname{Sp}(n) \cdot \operatorname{Sp}(1)$ acts on $\mathbb{F}^{n}$ by $A \cdot g(z)=A \cdot z \cdot g^{-1}$. Given an $\mathbb{R}$-vector subspace $V$ of $\mathbb{F}^{n}$, denote by $\operatorname{Sp}(V)$ the subgroup of $\operatorname{Sp}(n) \cdot \operatorname{Sp}(1)$ leaving $V$ invariant. Put $G=\nu^{-1}\left(V \rtimes\left(\operatorname{Sp}(V) \times \mathbb{R}^{+}\right)\right)$. Then $G$ is a closed subgroup of $\operatorname{Sim}(\mathcal{M})$ which preserves the subspace $\nu^{-1}(V)=\mathbb{R}^{3} \times V$. Note that $\mathcal{N}=\nu^{-1}(V)$ is also a nilpotent subgroup of $\mathcal{M}$. Suppose that $\operatorname{dim} V=k$. As $G$ stabilizes the point at infinity $\{\infty\}$, $G$ preserves the $(k+3)$-sphere $S^{k+3}=\left(\mathbb{R}^{3} \times V\right) \cup\{\infty\}$. Moreover, $G$ leaves invariant

$$
\mathcal{M}-\mathbb{R}^{3} \times V=\mathcal{M} \cup\{\infty\}-\mathbb{R}^{3} \times V \cup\{\infty\}=S^{4 n+3}-S^{k+3} .
$$

Let $X$ be the universal covering space of $S^{4 n+3}-S^{k+3}$.

LEMMA 3. Suppose that $V \neq \mathbb{F}^{n}$.

(1) $G$ acts properly on $S^{4 n+3}-S^{k+3}$.

(2) There is a G-invariant complete Riemannian metric on $S^{4 n+3}-S^{k+3}$.

(3) Given a discontinuous subgroup $\Gamma$ of $G$, let $(\tilde{\Gamma}, X)$ be any lift of the action $\left(\Gamma, S^{4 n+3}-S^{k+3}\right)$ to $X$. Then no such group $\tilde{\Gamma}$ acts properly discontinuously with compact quotient $X / \tilde{\Gamma}$.

Proof. (1) Let $K$ be a compact subset of $S^{4 n+3}-S^{k+3}$. We prove that the subset of $G, \zeta_{G}(K)=\{g \in G \mid g K \cap K \neq \emptyset\}$, is compact. There is an equivariant fibration:

$$
\mathbb{R}^{3} \rightarrow\left(G, S^{4 n+3}-S^{k+3}\right) \stackrel{\nu}{\longrightarrow}\left(V \rtimes\left(\mathrm{Sp}(V) \times \mathbb{R}^{+}\right), S^{4 n}-S^{k}\right),
$$

where $S^{k}=V \cup\{\infty\}$. Put $\hat{G}=V \rtimes\left(\operatorname{Sp}(V) \times \mathbb{R}^{+}\right)$. Let $\operatorname{Conf}\left(S^{4 n}\right)=\mathrm{PO}(4 n+1,1)$ be the group of conformal transformations of $S^{4 n}$. If $\operatorname{Conf}\left(S^{4 n}, S^{k}\right)$ is the subgroup of $\operatorname{Conf}\left(S^{4 n}\right)$ preserving $S^{k}$, then we have $\left(\operatorname{Conf}\left(S^{4 n}, S^{k}\right), S^{4 n}-S^{k}\right)=(\mathrm{PO}(k+1,1) \times$ $\left.\mathrm{O}(4 n-k), \mathbb{H}_{\mathbb{R}}^{k+1} \times S^{4 n-k-1}\right)$ where the product $\mathrm{PO}(k+1,1) \times \mathrm{O}(4 n-k)$ acts as isometries. (Compare [18].) Since $\hat{G}$ is a closed similarity subgroup of $\mathrm{PO}(k+1,1) \times$ $\mathrm{O}(4 n-k), \hat{G}$ acts properly on $S^{4 n}-S^{k}$. Let $\left\{g_{i}\right\}$ be a sequence in $\zeta_{G}(K)$. Given a sequence $\left\{x_{i}\right\} \in K$ with $\lim x_{i}=x$, suppose that $\lim g_{i} x_{i}=y$ for some $y \in K$. Since $\lim \nu\left(g_{i}\right) \nu\left(x_{i}\right)=\nu(y)$, there is an element $h \in \hat{G}$ such that $\lim \nu\left(g_{i}\right)=h$. As $\mathbb{R}^{3} \rightarrow G \stackrel{\nu}{\longrightarrow} \hat{G}$ is a principal fibration with contractible fiber, we choose a section $s: \hat{G} \longrightarrow G$. In particular, $\lim s\left(\nu\left(g_{i}\right)\right)=s(h)$. Let $s(h)=g^{\prime}$ so that $\lim s\left(\nu\left(g_{i}\right)\right) x_{i}=$ $g^{\prime} x$. Since there is a sequence $\left\{t_{i}\right\} \in \mathbb{R}^{3}$ for which $t_{i} \cdot s\left(\nu\left(g_{i}\right)\right)=g_{i}$, we have $\lim t_{i}$. $s\left(\nu\left(g_{i}\right)\right) x_{i}=y$. As $\mathbb{R}^{3}$ acts properly, there is an element $t \in \mathbb{R}^{3}$ such that $\lim t_{i}=t$. Hence $\lim g_{i}=\lim t_{i} \cdot s\left(\nu\left(g_{i}\right)\right)=t \cdot g^{\prime}$.

(2) There exists a $G$-invariant Riemannian metric $g$ on $S^{4 n+3}-S^{k+3}$ by (1). (Compare [27].) We prove that $g$ is complete. Let $\mathrm{d}$ be the distance function on $S^{4 n+3}-S^{k+3}$ and $\left\{x_{n}\right\}_{n \in \mathbb{N}}$ a Cauchy sequence in $S^{4 n+3}-S^{k+3}$. Take a simply connected closed subgroup $\nu^{-1}\left(V \rtimes \mathbb{R}^{+}\right)=\left(\mathbb{R}^{3} \times V\right) \rtimes \mathbb{R}^{+}$acting freely on $S^{4 n+3}-S^{k+3}$. 
Moreover, the solvable subgroup $V \rtimes \mathbb{R}^{+}\left(\subset \operatorname{Sim}(V)^{0}\right)$ acts simply transitively on $\mathbb{H}_{\mathbb{R}}^{k+1}$, the above fibration induces the following fibration:

$$
\left(\mathbb{R}^{3} \times V\right) \rtimes \mathbb{R}^{+} \rightarrow S^{4 n+3}-S^{k+3} \stackrel{\mu}{\longrightarrow} S^{4 n-k-1} .
$$

Then the sequence $\left\{\mu\left(x_{n}\right)\right\}$ has an accumulation point $z \in S^{4 n-k-1}$. Choose $\tilde{z} \in$ $S^{4 n+3}-S^{k+3}$ such that $\mu(\tilde{z})=z$. Let $K$ be a compact neighborhood of $\tilde{z}$ in $S^{4 n+3}-$ $S^{k+3}$. There is a number $\varepsilon>0$ such that the $\varepsilon$-ball $B_{\varepsilon}(\tilde{z})$ centered at $\tilde{z}$ is contained in $K$. As $\lim \mu\left(x_{n}\right)=z \in \mu(K)$, there exists a sufficiently large $L$ such that $\mu\left(x_{n}\right) \in$ $\mu\left(B_{\frac{\varepsilon}{3}}(\tilde{z})\right)$ for $n \geqq L$. Choose $\left\{\tilde{z}_{n}\right\} \in B_{\frac{\varepsilon}{3}}(\tilde{z})$ with $\mu\left(\tilde{z}_{n}\right)=\mu\left(x_{n}\right)$ for $n \geqq L$. Then there is a sequence $\left\{s_{n}\right\} \in\left(\mathbb{R}^{3} \times V\right) \rtimes \mathbb{R}^{+}$such that $x_{n}=s_{n} \cdot \tilde{z}_{n}$ for $n \geqq L$. As $\left\{x_{n}\right\}$ is Cauchy, there exists an $M>L$ such that $\mathrm{d}\left(x_{m}, x_{n}\right)<\frac{\varepsilon}{3}$ for $m, n \geqq \bar{M}$. In particular, $\mathrm{d}\left(x_{M}, x_{n}\right)=\mathrm{d}\left(s_{M} \cdot \tilde{z}_{M}, s_{n} \cdot \tilde{z}_{n}\right)<\frac{\varepsilon}{3}$. As $\mathrm{d}\left(\tilde{z}_{n}, \tilde{z}\right)<\frac{\varepsilon}{3}$ for $n \geqq M$,

$$
\begin{aligned}
\mathrm{d}\left(s_{n}^{-1} s_{M} \cdot \tilde{z}, \tilde{z}\right) & =\mathrm{d}\left(s_{M} \cdot \tilde{z}, s_{n} \tilde{z}\right) \\
& \leqq \mathrm{d}\left(s_{M} \cdot \tilde{z}, s_{M} \cdot \tilde{z}_{M}\right)+\mathrm{d}\left(s_{M} \cdot \tilde{z}_{M}, s_{n} \tilde{z}_{n}\right)+\mathrm{d}\left(s_{n} \tilde{z}_{n}, s_{n} \tilde{z}\right) \\
& =\mathrm{d}\left(\tilde{z}, \tilde{z}_{M}\right)+\mathrm{d}\left(x_{M}, x_{n}\right)+\mathrm{d}\left(\tilde{z}_{n}, \tilde{z}\right)<\varepsilon .
\end{aligned}
$$

Therefore $s_{n}^{-1} s_{M} \cdot \tilde{z} \in K$ for $n \geqq M$. By properness of $\left(\mathbb{R}^{3} \times V\right) \rtimes R^{+}$, we have $\lim s_{n}^{-1} s_{M}=s^{\prime}$ or $\lim s_{n}=s_{M} s^{\prime-1}$. As $\left\{\tilde{z}_{n}\right\} \in K$ and $K$ is compact, there is a point $w \in K$ with $\lim \tilde{z}_{n}=w$ up to a subsequence. Then,

$$
\lim x_{n}=\lim s_{n} \cdot \tilde{z}_{n}=s_{M} s^{\prime-1} \cdot w .
$$

Hence $S^{4 n+3}-S^{k+3}$ is complete.

(3) If $k \neq 4 n-2$, then $X=S^{4 n+3}-S^{k+3}$. The action $(\tilde{\Gamma}, X)$ coincides with the action $\left(\Gamma, S^{4 n+3}-S^{k+3}\right)$. Since $\hat{G}$ acts properly on $S^{4 n}-S^{k}=\mathbb{H}_{\mathbb{R}}^{k+1} \times S^{4 n-k-1}$ and transitively on $\mathbb{H}_{\mathbb{R}}^{k+1}$, the quotient $S^{4 n}-S^{k} / \hat{G}$ is compact Hausdorff. Noting the fibration that $G / \Gamma \longrightarrow X / \Gamma \longrightarrow X / G=S^{4 n}-S^{k} / \hat{G}$, if $X / \Gamma$ is compact, then $\Gamma$ is a discrete uniform subgroup of $G$. On the other hand, $G$ has the exact sequence $1 \rightarrow \mathbb{R}^{3} \times V \rightarrow G \stackrel{\tau}{\longrightarrow} \operatorname{Sp}(V) \times \mathbb{R}^{+}$in which $\mathcal{N}=\mathbb{R}^{3} \times V \subset \mathcal{M}$ is a maximal normal nilpotent Lie subgroup of $G$. If $\triangle=\Gamma \cap \mathcal{N}$, then $\triangle$ is a discrete uniform subgroup of $\mathcal{N}$. (See [37].) Thus $\tau(\Gamma)$ is discrete and cocompact in $\operatorname{Sp}(V) \times \mathbb{R}^{+}$. Since $\mathbb{R}^{+}$acts as contraction or expansion on $\mathcal{N} \subset \mathcal{M}$ (acts by different scale factors as in (1.2)), so does $\tau(\Gamma)$ on $\triangle$. Hence $\triangle$ cannot be discrete in $\mathcal{N}$, being a contradiction.

Suppose that $k=4 n-2$. Then $V=\mathbb{F}^{n-1} \times \mathbb{R}^{2} \subset \mathbb{F}^{n}$. So, the group $\operatorname{Sp}(V)$ is isomorphic to $\mathrm{Sp}(n-1) \cdot \mathrm{SO}(2)$ where $\mathrm{SO}(2)$ is a circle of $\mathrm{Sp}(1)$. Then the equivariant fibration of (1) induces the following:

$$
\mathbb{R}^{3} \rightarrow(\tilde{G}, X) \stackrel{\tilde{\nu}}{\longrightarrow}\left(V \rtimes\left(\operatorname{Sp}(n-1) \times \mathbb{R} \times \mathbb{R}^{+}\right), \mathbb{H}_{\mathbb{R}}^{4 n-1} \times \mathbb{R}\right),
$$

where $\mathbb{Z} \rightarrow \mathbb{R} \rightarrow \mathrm{SO}(2)$ is a covering group. As again the quotient space $X / \tilde{G}=\mathbb{H}_{\mathbb{R}}^{4 n-1} \times$ $\mathbb{R} / V \rtimes\left(\operatorname{Sp}(n-1) \times \mathbb{R} \times \mathbb{R}^{+}\right)=S^{4 n}-S^{4 n-2} / \hat{G}$ is compact Hausdorff. If $X / \tilde{\Gamma}$ is compact, then the fibration implies that $\tilde{\Gamma}$ is a discrete uniform subgroup of $\tilde{G}$. Since $\tilde{G}$ has the group extension $1 \rightarrow \mathbb{R}^{3} \times V \rightarrow \tilde{G} \stackrel{\tau}{\longrightarrow} \operatorname{Sp}(n-1) \times \mathbb{R} \times \mathbb{R}^{+} \rightarrow 1$ in which $\mathcal{N}=\mathbb{R}^{3} \times V$ is a maximal normal nilpotent Lie subgroup of $\tilde{G}$. Thus the intersection $\mathcal{N} \cap \tilde{\Gamma}$ is a discrete uniform subgroup of $\mathcal{N}$. The same argument yields a contradiction. Therefore there is no discrete cocompact subgroup $\tilde{\Gamma}$ of $\tilde{G}$. 
It is easy to see that the closed connected abelian subgroups of $\mathcal{M}$ are only $\mathbb{R}^{3}=\left(\mathbb{R}^{3}, 0\right), \mathbb{R}^{n}=\left(0, \mathbb{R}^{n}\right)$ or $\mathbb{R}^{3} \times \mathbb{R}^{n}$ up to conjugacy. The $\operatorname{subgroup}$ of $\operatorname{Sim}(\mathcal{M})$ leaving invariant $\mathbb{R}^{n}$ is isomorphic to the subgroup $\operatorname{Sim}\left(\mathbb{R}^{n}\right)^{0}=\mathbb{R}^{n} \rtimes\left(\operatorname{SO}(n) \times \mathbb{R}^{+}\right)$. When we take $V$ as the abelian group $\mathbb{R}^{n}, G=\mathbb{R}^{3} \rtimes \operatorname{Sim}\left(\mathbb{R}^{n}\right)^{0}$ which leaves invariant $\mathbb{R}^{3} \times \mathbb{R}^{n}$ and also $S^{n+3}$.

CoROLlaRY 4. The group $G$ acts properly on $\mathcal{M}-\mathbb{R}^{3} \times \mathbb{R}^{n}=S^{4 n+3}-S^{n+3}$. In particular, $\operatorname{Sim}\left(\mathbb{R}^{n}\right)$ acts properly.

Recall that a proper totally geodesic subspace in $\mathbb{H}_{\mathbb{F}}^{n+1}$ is isometric to $\mathbb{H}_{\mathbb{K}}^{m}$ ( $\mathbb{K}=$ $\mathbb{R}, \mathbb{C}$ or $\mathbb{F}, 1 \leqq m \leqq n$ ), or $\mathbb{H}_{R}^{n+1}, \mathbb{H}_{\mathbb{C}}^{n+1}$, or a 3-dimensional (hyperbolic) subspace $\mathbb{H}^{1}$ (I) (which is orthogonal to $\mathbb{H}_{\mathbb{R}}^{1}$ in $\mathbb{H}_{\mathbb{F}}^{1}$ ). (Compare [8].) In order to construct a non-homogeneous example but has compact quotient, we need the following.

Proposition 5.

(1) The subgroup of $\operatorname{PSp}(n+1,1)$ preserving $\mathbb{H}_{\mathbb{R}}^{n+1}$ in $\mathbb{H}_{\mathbb{F}}^{n+1}$ is isomorphic to $\mathrm{PO}(n+1,1) \times \mathrm{SO}(3)$, which also preserves $S^{n}$ in $S^{4 n+3}$.

(2) $\mathrm{PO}(n+1,1)$ acts properly on $S^{4 n+3}-S^{n}$.

(3) There is a complete Riemannian metric on $S^{4 n+3}-S^{n}$ invariant under $\mathrm{PO}(n+1,1) \times \mathrm{SO}(3)$.

(1) follows from the result of [8]; the subgroup of $\operatorname{Sp}(n+1,1)$ which preserves $\mathbb{H}_{\mathbb{R}}^{n+1}$ is isomorphic to $\mathrm{O}(n+1,1) \cdot \operatorname{Sp}(1)$. As before if $\operatorname{Conf}\left(S^{4 n+3}, S^{n}\right)$ is the subgroup of $\operatorname{Conf}\left(S^{4 n+3}\right)$ preserving $S^{n}=\partial H_{\mathbb{R}}^{n+1}$, then $\left(\operatorname{Conf}\left(S^{4 n+3}, S^{n}\right), S^{4 n+3}-S^{n}\right)=$ $\left(\mathrm{PO}(n+1,1) \times \mathrm{O}(3 n+3), \mathbb{H}_{\mathbb{R}}^{n+1} \times S^{3 n+2}\right)$ (cf. [18]). We have an isometric action of $\mathrm{PO}(n+1,1)$ on $S^{4 n+3}-S^{n}$. However it is noted that the above action of (2) on $S^{4 n+3}-S^{n}$ is different from this isometric action.

Proof. (2) Since $\mathbb{R}^{n} \cup\{\infty\}=S^{n} \subset \mathcal{M} \cup\{\infty\}=S^{4 n+3}$, note that $S^{4 n+3}-$ $S^{n}=\mathcal{M}-\mathbb{R}^{n}$. Moreover $\mathrm{PO}(n+1,1)=\mathrm{O}(n+1) \cdot \operatorname{Sim}\left(\mathbb{R}^{n}\right)$. It suffices to check that $\operatorname{Sim}\left(\mathbb{R}^{n}\right)$ acts properly on $\mathcal{M}-\mathbb{R}^{n}$. Let $K$ be a compact set of $\mathcal{M}-\mathbb{R}^{n}$ and $\zeta_{\operatorname{Sim}\left(\mathbb{R}^{n}\right)}(K)=\left\{g \in \operatorname{Sim}\left(\mathbb{R}^{n}\right) \mid g K \cap K \neq \emptyset\right\}$. Let $\left\{g_{i}\right\}$ be a sequence in $\zeta_{\operatorname{Sim}\left(\mathbb{R}^{n}\right)}(K)$. Given a sequence $\left\{p_{i}\right\} \in K$ with $\lim p_{i}=p$, suppose that $\lim g_{i} p_{i}=q$ for some $q \in K$. There is the fibration: $\mathbb{R}^{3} \rightarrow \mathcal{M}-\mathbb{R}^{3} \times \mathbb{R}^{n} \stackrel{\nu}{\longrightarrow} \mathbb{F}^{n}-\mathbb{R}^{n}$ as before.

Case I. Suppose that an infinite number of points $\left\{p_{i}\right\}$ satisfy that $\nu\left(p_{i}\right) \in \mathbb{R}^{n}$. Then we have $\nu(p) \in \mathbb{R}^{n}$. Recall that an element $g$ of $\operatorname{Sim}\left(\mathbb{R}^{n}\right)$ has the form:

$$
g=(0, x) \cdot(B, t)
$$

For

$$
\left(\begin{array}{c}
\alpha \\
z
\end{array}\right) \in \mathcal{M}
$$

we have

$$
g\left(\begin{array}{c}
\alpha \\
z
\end{array}\right)=(0, x) \cdot\left(\begin{array}{c}
t^{2} \alpha \\
t B z
\end{array}\right)=\left(\begin{array}{c}
t^{2} \alpha+\operatorname{Im}<x, t B z> \\
x+t B z
\end{array}\right)
$$

Let

$$
p_{i}=\left(\begin{array}{c}
\alpha_{i} \\
z_{i}
\end{array}\right) \rightarrow p=\left(\begin{array}{c}
\alpha \\
z
\end{array}\right)
$$


In our case, $z_{i}, z \in \mathbb{R}^{n}$. Note that $\alpha \neq 0=(0,0,0)$ because $p \in K$. In particular we have that $\lim \alpha_{i}^{-1}=\alpha^{-1}$. In the sequence

$$
g_{i}\left(\begin{array}{c}
\alpha_{i} \\
z_{i}
\end{array}\right)=\left(\begin{array}{c}
t_{i}^{2} \alpha_{i}+\operatorname{Im}<x_{i}, t_{i} B_{i} z_{i}> \\
x_{i}+t_{i} B_{i} z_{i}
\end{array}\right) \rightarrow q=\left(\begin{array}{c}
\alpha^{\prime} \\
z^{\prime}
\end{array}\right)
$$

since $\operatorname{Im}<x_{i}, t_{i} B_{i} z_{i}>=0$, this reduces to

$$
g_{i} p_{i}=\left(\begin{array}{c}
t_{i}^{2} \alpha_{i} \\
x_{i}+t_{i} B_{i} z_{i}
\end{array}\right)
$$

It follows that $\lim t_{i}^{2} \alpha_{i}=\alpha^{\prime}$. Then $t_{i}^{2}=t_{i}^{2} \cdot\left|\alpha_{i}\right| \cdot\left|\alpha_{i}^{-1}\right| \longrightarrow\left|\alpha^{\prime}\right| \cdot\left|\alpha^{-1}\right|$. Thus we assume that $\lim t_{i}=t<\infty$. As $\lim x_{i}+t_{i} B_{i} z_{i}=z^{\prime}, \lim z_{i}=z$ and $B_{i} \in \operatorname{SO}(n)$, we obtain that $\lim x_{i}=x$ for some $x \in \mathbb{R}^{n}$ (up to a subsequence). Assume $\lim B_{i}=B \in \operatorname{SO}(n)$. Then it follows that

$$
\lim g_{i}=\lim \left(0, x_{i}\right) \cdot\left(B_{i}, t_{i}\right)=(0, x) \cdot(B, t) \in \operatorname{Sim}\left(\mathbb{R}^{n}\right) .
$$

This proves Case I.

Case II. Suppose that an infinite number of points $\left\{p_{i}\right\}$ satisfy that $\nu\left(p_{i}\right) \in$ $\mathbb{F}^{n}-\mathbb{R}^{n}$ but $\nu(p) \in \mathbb{R}^{n}$. For the point $p_{i}$, put $z_{i}=y_{i}+\operatorname{Im}\left(z_{i}\right)$ where $y_{i}=\operatorname{Re}\left(z_{i}\right)$. As $\nu\left(p_{i}\right)=z_{i}$ converges to $\nu(p)=z$, it follows that $\lim y_{i}=z$ and $\lim \operatorname{Im}\left(z_{i}\right)=0$. Put

$$
p_{i}{ }^{\prime}=\left(\begin{array}{c}
\beta_{i} \\
\operatorname{Im}\left(z_{i}\right)
\end{array}\right)=\left(\begin{array}{c}
\alpha_{i}-\operatorname{Im}<y_{i}, z_{i}> \\
\operatorname{Im}\left(z_{i}\right)
\end{array}\right) \text {. }
$$

As $\operatorname{Im}<y_{i}, z_{i}>=<y_{i}, \operatorname{Im}\left(z_{i}\right)>\longrightarrow 0, \lim p_{i}{ }^{\prime}=(\alpha, 0)$. Since $\alpha \neq 0$, we may assume that $\beta_{i} \neq 0$ for infinitely many $i$. We have $\lim \left|\beta_{i}\right|^{-1}=|\alpha|^{-1}$. Let $L_{y_{i}}=\left(0, y_{i}\right) \cdot(\mathrm{I}, 1) \in$ $\operatorname{Sim}\left(\mathbb{R}^{n}\right)$ be the translation. Then $\lim L_{y_{i}}=(0, z) \cdot\left([, 1)=L_{z} \in \operatorname{Sim}\left(\mathbb{R}^{n}\right)\right.$. Consider the sequence $\left\{g_{i} \circ L_{y_{i}}\right\} \in \operatorname{Sim}\left(\mathbb{R}^{n}\right)$. Then,

$$
g_{i} \circ L_{y_{i}}\left(p_{i}{ }^{\prime}\right)=g_{i}\left(\begin{array}{c}
\alpha_{i}-\operatorname{Im}<y_{i}, z_{i}>+<y_{i}, \operatorname{Im}\left(z_{i}\right)> \\
y_{i}+\operatorname{Im}\left(z_{i}\right)
\end{array}\right)=g_{i}\left(\begin{array}{c}
\alpha_{i} \\
z_{i}
\end{array}\right)=g_{i} p_{i} \longrightarrow q .
$$

On the other hand, $g_{i} \circ L_{y_{i}}=\left(0, x_{i}\right) \cdot\left(B_{i}, t_{i}\right) \circ\left(0, y_{i}\right)=\left(0, x_{i}+t_{i} B_{i} y_{i}\right) \cdot\left(B_{i}, t_{i}\right)$. Put $g_{i} \circ L_{y_{i}}=\left(0, w_{i}\right) \cdot\left(B_{i}, t_{i}\right)$. Then

$$
g_{i} \circ L_{y_{i}}\left(p_{i}{ }^{\prime}\right)=\left(\begin{array}{c}
t_{i}^{2} \cdot \beta_{i}+<w_{i}, t_{i} B_{i} \operatorname{Im}\left(z_{i}\right)> \\
w_{i}+t_{i} B_{i} \operatorname{Im}\left(z_{i}\right)
\end{array}\right) .
$$

It follows that

$$
\lim \operatorname{Re}\left(w_{i}+t_{i} B_{i} \operatorname{Im}\left(z_{i}\right)\right)=\operatorname{Re}\left(z^{\prime}\right), \text { i.e., } w_{i} \longrightarrow \operatorname{Re}\left(z^{\prime}\right) .
$$

Similarly, $\operatorname{Im}\left(w_{i}+t_{i} B_{i} \operatorname{Im}\left(z_{i}\right)\right)=t_{i} B_{i} \operatorname{Im}\left(z_{i}\right) \longrightarrow \operatorname{Im}\left(z^{\prime}\right)$. Therefore,

$$
\lim <w_{i}, t_{i} B_{i} \operatorname{Im}\left(z_{i}\right)>=<\operatorname{Re}\left(z^{\prime}\right), \operatorname{Im}\left(z^{\prime}\right)>.
$$

Since $\lim \left(t_{i}^{2} \cdot \beta_{i}+<w_{i}, t_{i} B_{i} \operatorname{Im}\left(z_{i}\right)>\right)=\alpha^{\prime}$,

$$
t_{i}^{2}=t_{i}^{2} \cdot\left|\beta_{i}\right| \cdot\left|\beta_{i}\right|^{-1} \leqq\left(\left|\alpha^{\prime}\right|+\left|<\operatorname{Re}\left(z^{\prime}\right), \operatorname{Im}\left(z^{\prime}\right)>\right|\right) \cdot|\alpha|^{-1}+1 .
$$

Thus, $\left\{t_{i}\right\}$ is bounded. Let $\lim t_{i}=t$ (up to a subsequence). 
As $g_{i} \circ L_{y_{i}}=\left(0, w_{i}\right) \cdot\left(B_{i}, t_{i}\right) \longrightarrow\left(0, \operatorname{Re}\left(z^{\prime}\right)\right) \cdot(B, t)$, and $L_{y_{i}} \longrightarrow L_{z}$, $\lim g_{i}=\lim g_{i} \circ L_{y_{i}} \circ L_{y_{i}}^{-1}=\left(0, \operatorname{Re}\left(z^{\prime}\right)\right) \cdot(B, t) \circ L_{z}^{-1} \in \operatorname{Sim}\left(\mathbb{R}^{n}\right)$.

So $\left\{g_{i}\right\}$ converges.

Case III. Suppose that an infinite number of points $\left\{p_{i}\right\}$ satisfy that $\nu\left(p_{i}\right) \in$ $\mathbb{F}^{n}-\mathbb{R}^{n}$ and $\nu(p) \in \mathbb{F}^{n}-\mathbb{R}^{n}$. Then $\operatorname{Im}(z)=\operatorname{Im}(\nu(p)) \neq 0$. Recall

$$
g_{i} p_{i}=\left(\begin{array}{c}
t_{i}^{2} \alpha_{i}+\operatorname{Im}<x_{i}, t_{i} B_{i} z_{i}> \\
x_{i}+t_{i} B_{i} z_{i}
\end{array}\right) .
$$

If $x_{i}+t_{i} B_{i} z_{i} \in \mathbb{R}^{n}$, then $\nu\left(p_{i}\right)=z_{i} \in \mathbb{R}^{n}$. In our case $g_{i} p_{i} \in \mathcal{M}-\mathbb{R}^{3} \times \mathbb{R}^{n}$.

If $\lim g_{i} p_{i}=q \in \mathcal{M}-\mathbb{R}^{3} \times \mathbb{R}^{n}$, then all points $\left\{p_{i}, p, g_{i} p_{i}, q\right\} \in \mathcal{M}-\mathbb{R}^{3} \times \mathbb{R}^{n}=$ $S^{4 n+3}-S^{n+3}$. By Corollary $4, \operatorname{Sim}\left(\mathbb{R}^{n}\right)$ acts properly on $\mathcal{M}-\mathbb{R}^{3} \times \mathbb{R}^{n}$. It follows that $\lim g_{i}=g \in \operatorname{Sim}\left(\mathbb{R}^{n}\right)$.

We show that $q$ does not lie in $\mathbb{R}^{3} \times \mathbb{R}^{n}$. Suppose that $q \in \mathbb{R}^{3} \times \mathbb{R}^{n}$. Then $z^{\prime} \in \mathbb{R}^{n}$ or $\operatorname{Im}\left(z^{\prime}\right)=0$. As $q \in K \subset \mathcal{M}-\mathbb{R}^{n}, \alpha^{\prime} \neq 0$. Since $g_{i} p_{i} \longrightarrow q$, note that $t_{i}^{2} \alpha_{i}+\operatorname{Im}<x_{i}, t_{i} B_{i} z_{i}>\longrightarrow \alpha^{\prime}$ and $x_{i}+t_{i} B_{i} z_{i} \longrightarrow z^{\prime}$. It follows that $\operatorname{Im}\left(x_{i}+\right.$ $\left.t_{i} B_{i} z_{i}\right)=t_{i} B_{i} \operatorname{Im}\left(z_{i}\right) \longrightarrow \operatorname{Im}\left(z^{\prime}\right)=0$. Then, $\lim \left|t_{i} B_{i} \operatorname{Im}\left(z_{i}\right)\right|=\lim t_{i}\left|\operatorname{Im}\left(z_{i}\right)\right|=0$. On the other hand, $\nu\left(p_{i}\right)=z_{i} \longrightarrow \nu(p)=z$, so $\lim \operatorname{Im}\left(z_{i}\right)=\operatorname{Im}(z) \neq 0$ by our case. Thus $\lim \left|\operatorname{Im}\left(z_{i}\right)\right|^{-1}=|\operatorname{Im}(z)|^{-1}$. Therefore, $\lim t_{i}=\lim t_{i}\left|\operatorname{Im}\left(z_{i}\right)\right| \cdot\left|\operatorname{Im}\left(z_{i}\right)\right|^{-1}=$ $0 \cdot|\operatorname{Im}(z)|^{-1}=0$. Moreover,

$$
\begin{aligned}
\left|x_{i}\right| & =\left|x_{i}+t_{i} B_{i} z_{i}-t_{i} B_{i} z_{i}\right| \\
& \leqq\left|x_{i}+t_{i} B_{i} z_{i}\right|+t_{i}\left|z_{i}\right| \\
& \leqq\left|z^{\prime}\right|+0 \cdot|z|+1=\left|z^{\prime}\right|+1 .
\end{aligned}
$$

So $\left\{x_{i}\right\}$ is bounded, let $\lim x_{i}=x$. Then

$$
t_{i}^{2} \alpha_{i}+\operatorname{Im}<x_{i}, t_{i} B_{i} z_{i}>=t_{i}^{2} \alpha_{i}+<x_{i}, t_{i} B_{i} \operatorname{Im}\left(z_{i}\right)>\longrightarrow 0 \cdot \alpha+<x, 0>=0 .
$$

This contradicts that $t_{i}^{2} \alpha_{i}+\operatorname{Im}<x_{i}, t_{i} B_{i} z_{i}>\longrightarrow \alpha^{\prime} \neq 0$ as above.

(3) There exist a $\mathrm{PO}(n+1,1) \times \mathrm{SO}(3)$-invariant Riemannian metric $g$ on $S^{4 n+3}-S^{n}$ by (2) and a principal fibration $\mathbb{R}^{n} \rtimes \mathbb{R}^{+} \rightarrow S^{4 n+3}-S^{n} \stackrel{\mu}{\longrightarrow} S^{4 n+3}-S^{n} / \mathbb{R}^{n} \rtimes \mathbb{R}^{+}$. Since $S^{4 n+3}-S^{n} \approx \mathbb{H}_{\mathbb{R}}^{n+1} \times S^{3 n+2}$ topologically and $\mathbb{R}^{n} \rtimes \mathbb{R}^{+} \subset \operatorname{Sim}\left(\mathbb{R}^{n}\right)$ is a transitive subgroup of $\mathbb{H}_{\mathbb{R}}^{n+1}, S^{4 n+3}-S^{n} / \mathbb{R}^{n} \rtimes \mathbb{R}^{+}$is compact (which is homeomorphic to $S^{3 n+2}$ ). Then as in the argument of (2) of Lemma 3 , we can prove that $g$ is complete.

The same argument of Proposition 5 can be applied to the complex case. Combined with Corollary 4, we obtain the following.

Corollary 6.

(1) The subgroup of $\operatorname{PSp}(n+1,1)$ preserving $\mathbb{H}_{\mathbb{C}}^{n+1}$ in $\mathbb{H}_{\mathbb{F}}^{n+1}$ is isomorphic to $P\left(\mathrm{U}(n+1,1) \cdot S^{1}\{ \pm 1, \pm j\}\right) \approx \mathrm{U}(n+1,1) \times\{ \pm 1\}$, which preserves also $S^{2 n+1}$ in $S^{4 n+3}$.

(2) Put $H=P\left(\mathrm{U}(n+1,1) \cdot S^{1}\{ \pm 1, \pm j\}\right)$. Then $H$ acts properly on $S^{4 n+3}-S^{2 n+1}$. Moreover, $S^{4 n+3}-S^{2 n+1}$ admits an $H$-invariant complete Riemannian metric.

(3) There is a compact spherical pseudo-quaternionic manifold $S^{4 n+3}-S^{n} / \Gamma$ for which $S^{4 n+3}-S^{n}$ is not homogeneous but $\Gamma$ is a discrete uniform subgroup of $\mathrm{PO}(n+1,1)$. Similarly $S^{4 n+3}-S^{2 n+1}$ is not homogeneous but has the compact quotient $S^{4 n+3}-S^{2 n+1} / \Gamma$ for a discrete uniform subgroup $\Gamma$ of $\mathrm{U}(n+1,1)$. 
Note 7. Let $\mathbb{H}_{\mathbb{R}}^{n} \subset \mathbb{H}_{\mathbb{R}}^{n+1} \subset \mathbb{H}_{\mathbb{F}}^{n+1}$ be the canonical inclusion of totally geodesic real hyperbolic subspaces. We have also the embedding: $\mathbb{H}_{\mathbb{R}}^{n} \subset \mathbb{H}_{\mathbb{C}}^{n} \subset \mathbb{H}_{\mathbb{F}}^{n}$ by taking its span. The subgroup of $\operatorname{PSp}(n+1,1)=\operatorname{Iso}\left(\mathbb{H}_{\mathbb{F}}^{n+1}\right)$ preserving $\mathbb{H}_{\mathbb{R}}^{n}$ is isomorphic to $(\mathrm{O}(n, 1) \cdot \operatorname{Sp}(1)) \cdot \operatorname{Sp}(1)=\operatorname{Iso}\left(\mathbb{H}_{\mathbb{F}}^{n+1}, \mathbb{H}_{\mathbb{R}}^{n}\right)$. Since those elements also leave the span $\mathbb{H}_{\mathbb{F}}^{n}$ invariant, Iso $\left(\mathbb{H}_{\mathbb{F}}^{n+1}, \mathbb{H}_{\mathbb{R}}^{n}\right)$ is a subgroup of $\operatorname{Iso}\left(\mathbb{H}_{\mathbb{F}}^{n+1}, \mathbb{H}_{\mathbb{F}}^{n}\right)=\operatorname{Sp}(n, 1) \cdot \operatorname{Sp}(1)$. On the other hand, let $\mathbb{Z} / 2 \cdot \operatorname{Sp}(1)=\operatorname{Sp}(1)$ be the subgroup of Iso $\left(\mathbb{H}_{\mathbb{F}}^{n+1}, \mathbb{H}_{\mathbb{R}}^{n}\right)$. Then we note that $\operatorname{Sp}(1)$ fixes $\mathbb{H}_{\mathbb{R}}^{n}$ pointwisely. So does its span $\mathbb{H}_{\mathbb{F}}^{n}$, but rotates $\mathbb{H}_{\mathbb{R}}^{n+1}$ with axis $\mathbb{H}_{\mathbb{R}}^{n}$. Hence it is possible to bend $\mathbb{H}_{\mathbb{R}}^{n+1}$ along $\mathbb{H}_{\mathbb{R}}^{n}$ inside $\mathbb{H}_{\mathbb{F}}^{n+1}$ equivariantly with respect to a discrete uniform subgroup $\Gamma$ of $\mathrm{PO}(n+1,1)$. In fact, a compact manifold $M_{1}=S^{4 n+3}-S^{n} / \Gamma$ of Corollary 6 admits a spherical pseudo-quaternionic structure $\left(\operatorname{dev}_{t}, \rho_{t}\right)$ for an element $t \in \operatorname{Sp}(1)$. For $t$ sufficiently close to 1 , the nearby structure theorem by Thurston (cf. [42]) implies that the holonomy representation $\rho_{t}: \Gamma \rightarrow \operatorname{PSp}(n+1,1)$ is discrete faithful and the developing image $\operatorname{dev}\left(\tilde{M}_{1}\right)=S^{4 n+3}-$ $L\left(\rho_{t}(\Gamma)\right)$. (See Remark 31.)

\section{Quaternionic Carnot-Carathéodory geometry.}

2.1. Sasakian 3-structure on $S^{4 n+3}$. Recall the construction of Sasakian 3structure on $S^{4 n+3}$. (Compare [40],[41].) Denote by $<>$ the Hermitian inner product over $\mathbb{F}^{n+1}$, which is invariant under the standard quaternionic structure $\{I, J, K\}$. Let $<,>_{p}$ be the inner procluct on $\mathrm{T}_{p} \mathbb{F}^{n+1}$ obtained from the parallel translation of the inner product $<,>_{0}=<,>$ at the origin of $\mathbb{F}^{n+1}$. Letting $g_{p}(X, Y)=\operatorname{Re}<X, Y>_{p}$ for $X, Y \in \mathrm{T}_{p} \mathbb{F}^{n+1}, g$ is the standard euclidean metric on $\mathbb{F}^{n+1}$ which is invariant under $\{I, J, K\}$. Let $S^{4 n+3}$ be the unit sphere in $\mathbb{F}^{n+1}$. The restriction $g$ to $S^{4 n+3}$ gives the spherical Riemannian metric on $S^{4 n+3}$. There exists a normal vector field $N$ on $S^{4 n+3}$ such that $T S^{4 n+3} \oplus N=\mathrm{TF}^{n+1} \mid S^{4 n+3}$. Put

$$
\xi_{1}=I N, \quad \xi_{2}=J N, \quad \xi_{3}=K N .
$$

Then the subspace generated by $\left\{\xi_{i}\right\}_{i=1,2,3}$ with $N$ forms the tangent plane $\mathrm{TF}^{1}$ in $\mathrm{TF}^{n+1}$. Since $g\left(\xi_{1}, N\right)=\operatorname{Re}\langle I N, N\rangle=0$, similarly for $J, K$, the subspace $\left\{\xi_{i}\right\}_{i=1,2,3}$ belongs to $T S^{4 n+3}$. The full set $\left(S^{4 n+3}, g,\left\{\xi_{1}, \xi_{2}, \xi_{3}\right\},\{I, J, K\}\right)$ is said to be the canonical Sasakian 3-structure on $S^{4 n+3}$. It is easy to check that the isometry group $\operatorname{Iso}\left(S^{4 n+3}, g\right)^{0}$ is isomorphic to $\operatorname{Sp}(n+1) \cdot \operatorname{Sp}(1)$.

Identify $\operatorname{Im} \mathbb{F}=\mathbb{R} i+\mathbb{R} j+\mathbb{R} k$ with the Lie algebra $\mathfrak{s p}(1)$ of $\operatorname{Sp}(1)$. Put $\omega_{i}(X)=$ $g\left(\xi_{i}, X\right)$. Then $\omega_{i}$ is a (real valued) 1 -form on $S^{4 n+3}$. Define an $\mathfrak{s p}(1)$-valued 1 -form $\omega$ on $S^{4 n+3}$ to be

$$
\omega(X)=\omega_{1}(X) \boldsymbol{i}+\omega_{2}(X) \boldsymbol{j}+\omega_{3}(X) \boldsymbol{k} .
$$

A direct calculation shows that for $X, Y \in T S^{4 n+3}$

$$
d \omega(X, Y)=g(X, I Y) i+g(X, J Y) j+g(X, K Y) \boldsymbol{k} .
$$

If $R_{a}: S^{4 n+3} \rightarrow S^{4 n+3}$ is the right translation defined by $R_{a}(x)=x \cdot a$ for $a \in \operatorname{Sp}(1)$, then $\omega$ satisfies that $R_{a}^{*} \omega=\bar{a} \cdot \theta \cdot a$ (cf. [41]). Thus $\omega$ turns out to be a connection form of the Hopf bundle:

$$
\mathrm{Sp}(1) \rightarrow S^{4 n+3} \stackrel{\nu}{\longrightarrow} \mathbb{F P}^{n}
$$

Put

$$
B=\left\{X \in T S^{4 n+3} \mid \omega_{i}(X)=0 \text { for } i=1,2,3\right\}
$$


Since $g \mid B \times B$ is invariant under $\{I, J, K\}, B$ is a $4 n$-dimensional invariant subbundle of $T S^{4 n+3}$ such that

$$
B \oplus\left\{\xi_{1}, \xi_{2}, \xi_{3}\right\}=T S^{4 n+3} .
$$

By $(*)$, we have $[B, B]=\left\{\xi_{1}, \xi_{2}, \xi_{3}\right\}$ so that $B$ is a Carnot-Carathéodory structure on $S^{4 n+3}$ by the definition (cf. [32]).

We examine further properties on the Carnot-Carathéodory structure $B$ on $S^{4 n+3}$ from the conformal viewpoint.

LEMMA 8.

(I) (nondegenerate) The form $\omega$ satisfies that

$$
\omega \wedge \omega \wedge \omega \wedge \overbrace{d \omega^{2} \wedge \cdots \wedge d \omega^{2}}^{n \text { times }} \neq 0 \text { at every point of } S^{4 n+3} .
$$

(II) (integrable) There exist quaternion valued one-forms $\omega^{\alpha}(\alpha=1, \ldots, n)$ on $S^{4 n+3}$ such that

$$
d \omega=-\frac{1}{2} \delta_{\bar{\alpha} \beta} \omega^{\bar{\alpha}} \wedge \omega^{\beta} \quad(\bmod \omega)
$$

Proof. (I) If $\omega=\omega_{1} \boldsymbol{i}+\omega_{2} \boldsymbol{j}+\omega_{3} \boldsymbol{k}$, then the three-form $\omega \wedge \omega \wedge \omega$ and the four-form $d \omega \wedge d \omega$ are real valued;

$$
\omega^{3}=-6 \omega_{1} \wedge \omega_{2} \wedge \omega_{3}, \quad d \omega^{2}=-\left(d \omega_{1} \wedge d \omega_{1}+d \omega_{2} \wedge d \omega_{2}+d \omega_{3} \wedge d \omega_{3}\right) .
$$

Choose an orthonormal vector field $X_{\alpha}(\alpha=1, \ldots, n)$ from $B$ with respect to $g$ so that $\left\{X_{\alpha}, I X_{\alpha}, J X_{\alpha}, K X_{\alpha}\right\}_{\alpha=1, \ldots, n}$ forms a basis of $B$. Then the nonzero terms are

$$
\begin{aligned}
d \omega_{1}\left(X_{\alpha}, I X_{\alpha}\right) & =d \omega_{1}\left(J X_{\alpha}, K X_{\alpha}\right)=d \omega_{2}\left(X_{\alpha}, J X_{\alpha}\right)=d \omega_{2}\left(K X_{\alpha}, I X_{\alpha}\right) \\
& =d \omega_{3}\left(X_{\alpha}, K X_{\alpha}\right)=d \omega_{3}\left(J X_{\alpha}, K X_{\alpha}\right)=-1,
\end{aligned}
$$

and so $d \omega_{1}^{2}\left(X_{\alpha}, I X_{\alpha}, J X_{\alpha}, K X_{\alpha}\right)=-\frac{1}{3}$. Then a calculation shows that

$$
d \omega_{1}^{2 p}\left(X_{1}, I X_{1}, J X_{1}, K X_{1} ; \ldots ; X_{n}, I X_{n}, J X_{n}, K X_{n}\right)=\frac{(2 p) ! \cdot 2^{2 p}}{(4 p) !}
$$

and $\omega_{1} \wedge \omega_{2} \wedge \omega_{3}\left(\xi_{1}, \xi_{2}, \xi_{3}\right)=\frac{1}{6}$. We have

$$
\begin{aligned}
\omega^{3} \wedge d \omega^{2 n} & =6 \omega_{1} \wedge \omega_{2} \wedge \omega_{3} \wedge\left(d \omega_{1}^{2}+d \omega_{2}{ }^{2}+d \omega_{3}^{2}\right)^{n} \\
& =\sum_{p+q+r=n} \frac{n !}{p ! q ! r !} \cdot \frac{(2 p) ! \cdot 2^{2 p}}{(4 p) !} \cdot \frac{(2 q) ! \cdot 2^{2 q}}{(4 q) !} \cdot \frac{(2 r) ! \cdot 2^{2 r}}{(4 r) !}
\end{aligned}
$$

which is a positive constant.

$(I I)$ Choose a coframe $\theta^{\alpha}$ with $\theta^{\alpha}\left(X_{\beta}\right)=\delta_{\beta}^{\alpha}(\alpha, \beta=1, \ldots, n)$. Put

$$
\theta^{\alpha+n}=-\theta^{\alpha} \circ I, \theta^{\alpha+2 n}=-\theta^{\alpha} \circ J, \theta^{\alpha+3 n}=-\theta^{\alpha} \circ K,
$$


and define a quaternion valued one-form $\omega_{\alpha}$ to be $\omega^{\alpha}==\theta^{\alpha}+\theta^{\alpha+n} i+\theta^{\alpha+2 n} j+\theta^{\alpha+3 n} k$. As $B \oplus\left\{\xi_{1}, \xi_{2}, \xi_{3}, N\right\}=B \oplus \mathrm{TF}^{1}=\mathrm{TF} \mathbb{F}^{n+1} \mid S^{4 n+3}$, we have that $\langle>| B=\sum_{\alpha} \omega^{\bar{\alpha}} \otimes \omega^{\alpha}$. If we note $g(X, Y)=\operatorname{Re}\langle X, Y\rangle$, then each $d \omega_{1}(X, Y)=g(X, I Y), d \omega_{2}(X, Y)=$ $g(X, J Y)$ or $d \omega_{3}(X, Y)=g(X, K Y)$ represents the imaginary part of $\sum_{\alpha} \omega^{\bar{\alpha}} \otimes \omega^{\alpha}(X, Y)$ for $X, Y \in B$ respectively. A calculation shows that

$$
\begin{aligned}
& d \omega_{1}=-\sum_{\alpha}\left(\theta^{\alpha} \wedge \theta^{\alpha+n}-\theta^{\alpha+2 n} \wedge \theta^{\alpha+3 n}\right) \\
& d \omega_{2}=-\sum_{\alpha}\left(\theta^{\alpha} \wedge \theta^{\alpha+2 n}-\theta^{\alpha+3 n} \wedge \theta^{\alpha+n}\right) \\
& d \omega_{3}=-\sum_{\alpha}\left(\theta^{\alpha} \wedge \theta^{\alpha+3 n}-\theta^{\alpha+n} \wedge \theta^{\alpha+2 n}\right) .
\end{aligned}
$$

On the other hand,

$$
\begin{aligned}
& \frac{1}{2} \delta_{\bar{\alpha} \beta} \omega^{\bar{\alpha}} \wedge \omega^{\beta}=\frac{1}{2} \sum_{\alpha} \omega^{\bar{\alpha}} \wedge \omega^{\alpha} \\
= & \sum_{\alpha}\left(\theta^{\alpha} \wedge \theta^{\alpha+n}-\theta^{\alpha+2 n} \wedge \theta^{\alpha+3 n}\right) i+\sum_{\alpha}\left(\theta^{\alpha} \wedge \theta^{\alpha+2 n}-\theta^{\alpha+3 n} \wedge \theta^{\alpha+n}\right) j \\
& +\sum_{\alpha}\left(\theta^{\alpha} \wedge \theta^{\alpha+3 n}-\theta^{\alpha+n} \wedge \theta^{\alpha+2 n}\right) k .
\end{aligned}
$$

Therefore, we obtain that $d \omega=-\frac{1}{2} \delta_{\bar{\alpha} \beta} \omega^{\bar{\alpha}} \wedge \omega^{\beta}(\bmod \omega)$.

Definition 9. We call the bundle $B$ a quaternionic Carnot-Carathéodory structure on $S^{4 n+3}$. An $\mathfrak{s p}(1)$-valued one-form $\eta$ on $S^{4 n+3}$ represents the same quaternionic Carnot-Carathéodory structure if $\eta=\bar{\lambda} \cdot \omega \cdot \lambda$ for some nonzero function $\lambda: S^{4 n+3} \longrightarrow \mathbb{F}^{*}$.

A diffeomorphism $f: S^{4 n+3} \rightarrow S^{4 n+3}$ is called a quaternionic Carnot-Carathéodory transformation if $f^{*} \omega=\bar{\lambda} \cdot \omega \cdot \lambda$ for some nonzero function $\lambda: S^{4 n+3} \rightarrow \mathbb{F}^{*}$.

Let $\lambda=u \cdot \mu$ where $u: S^{4 n+3} \rightarrow \mathbb{R}^{+}$is a positive function and $\mu: S^{4 n+3} \rightarrow \operatorname{Sp}(1)$ is a function, and $\mu \rightarrow A$ under the map $\mathrm{Sp}(1) \longrightarrow \operatorname{Aut}(\mathbb{F})=\mathrm{SO}(3)$, i.e., $(\bar{\mu} \boldsymbol{i} \mu, \bar{\mu} \boldsymbol{j} \mu, \bar{\mu} \boldsymbol{k} \mu)=$ $(i, j, k) \cdot A$. Equivalently, a quaternionic Carnot-Carathéodory transformation $f$ is a diffeomorphism satisfying that $f_{*}$ preserves $B$, and $f_{*}\left(\xi_{1}, \xi_{2}, \xi_{3}\right)=u^{2} \cdot\left(\xi_{1}, \xi_{2}, \xi_{3}\right) \cdot A(p)$ $(\bmod B)_{p}$ at each point $p \in S^{4 n+3}$.

Denote $\operatorname{Aut}_{\mathrm{QCC}}\left(S^{4 n+3}\right)$ the group of all quaternionic Carnot-Carathéodory transformations of $S^{4 n+3}$ onto itself.

\subsection{Quaternionic Carnot-Carathéodory structure.} $\mathbb{F}^{n+1}$.

Let $S^{4 n+3}=\left\{\left.\left(q_{0}, \cdots, q_{n}\right) \in \mathbb{F}^{n+1}|| q_{0}\right|^{2}+\cdots+\left|q_{n}\right|^{2}=1\right\}$ be the unit sphere in

LEMma 10. Let $\theta=\bar{q}_{0} d q_{0}+\cdots+\bar{q}_{n} d q_{n}$ be an $\mathfrak{s p}(1)$-valued 1 -form on $S^{4 n+3}$. Then $\theta=\omega$ on $S^{4 n+3}$.

Proof. By the definition, $\quad \xi_{1}=\left(q_{0} i, \cdots, q_{n} \boldsymbol{i}\right), \quad \xi_{2}=\left(q_{0} \boldsymbol{j}, \cdots, q_{n} \boldsymbol{j}\right), \quad \xi_{3}=$ $\left(q_{0} k, \cdots, q_{n} k\right)$ at a point $q=\left(q_{0}, \cdots, q_{n}\right)$. Let $\vec{x}=\left(x_{0}, \cdots, x_{n}\right)$ be the vector parallel to a tangent vector $X \in T_{q} S^{4 n+3}$. As $g_{q}(N, X)=\operatorname{Re}<\vec{q}, \vec{x}>=0$, we can put $\overline{q_{0}} \cdot x_{0}+\cdots+\overline{q_{n}} \cdot x_{n}=a i+b j+c k$. 
Since $\left\langle\xi_{1}, X>=-i\left(\overline{q_{0}} \cdot x_{0}+\cdots+\overline{q_{n}} \cdot x_{n}\right)\right.$, similarly for $\xi_{2}, \xi_{3}$, a calculation shows that $\operatorname{Re}\left\langle\xi_{1}, X>=a, \operatorname{Re}\left\langle\xi_{2}, X>=b, \operatorname{Re}\left\langle\xi_{3}, X>=c\right.\right.\right.$. As $\omega(X)=$ $\operatorname{Re}\left\langle\xi_{1}, X>i+\operatorname{Re}\left\langle\xi_{2}, X>j+\operatorname{Re}\left\langle\xi_{3}, X>k\right.\right.\right.$ from (2.1), it follows that $\omega(X)=\overline{q_{0}} \cdot x_{0}+\cdots+\overline{q_{n}} \cdot x_{n}$. On the other hand, if $\left\{e_{0}, \cdots, e_{n}\right\}$ is dual to the forms $\left\{d q_{0}, \cdots, d q_{n}\right\}$, then $X=e_{0} \cdot x_{0}+\cdots+e_{n} \cdot x_{n}$ which implies that $\theta(X)=$ $\overline{q_{0}} \cdot x_{0}+\cdots+\overline{q_{n}} \cdot x_{n}$.

Let $V_{0}^{4 n+7}=\left\{z \in \mathbb{F}^{n+2}-\left.\{0\}|-| z_{1}\right|^{2}+\left|z_{2}\right|^{2}+\cdots+\left|z_{n+2}\right|^{2}=0\right\}$ be the quadric as before. Define an $\mathfrak{s p}(1)$-valued one-form on $V_{0}^{4 n+7}\left(\subset \mathbb{F}^{n+2}-\{0\}\right)$ to be

$$
\tilde{\theta}=\bar{z}_{1}^{-1}\left(-\bar{z}_{1} d z_{1}+\bar{z}_{2} d z_{2}+\cdots+\bar{z}_{n+2} d z_{n+2}\right) z_{1}^{-1} .
$$

If $\pi: V_{0}^{4 n+7} \longrightarrow S^{4 n+3}$ is the projection defined by $\pi\left(z_{1}, \ldots, z_{n+2}\right)=\left(z_{2} \cdot z_{1}^{-1}, \ldots, z_{n+2} \cdot z_{1}^{-1}\right)$, it is easy to check that $\pi^{*} \theta=\tilde{\theta}$ on $V_{0}^{4 n+7}$. Note from $(1.2)$ that $P\left(f_{1}\right)=\infty$ where

$$
f_{1}=\left(e_{1}+e_{n+2}\right) / \sqrt{2}=\left(\begin{array}{c}
\frac{1}{\sqrt{2}} \\
0 \\
\vdots \\
0 \\
\frac{1}{\sqrt{2}}
\end{array}\right)
$$

Thus $\pi\left(f_{1}\right)=(0, \ldots, 0,1)$ which is identified with $\{\infty\}$.

LEMMA 11.

(i) $\operatorname{PSp}(n+1,1) \subset \operatorname{Aut}_{\mathrm{QCC}}\left(S^{4 n+3}\right)$.

(ii) For an arbitrary $a \in \mathbb{F}^{*}$, there exists an element $g \in \operatorname{Sp}(1) \times \mathbb{R}^{+} \subset \operatorname{PSp}(n+1,1)_{\infty}$ such that $g^{*} \omega=\bar{\chi} \cdot \omega \cdot \chi$ for some function $\chi: S^{4 n+3} \rightarrow \mathbb{F}^{*}$ with $\chi(\infty)=a^{-1}$.

Proof. (i) Let $f \in \operatorname{PSp}(n+1,1)$ be an element. If $\tilde{f}: V_{0}^{4 n+7} \longrightarrow V_{0}^{4 n+7}$ is a lift of $f$ to $\operatorname{Sp}(n+1,1)$, then $\tilde{f}$ is represented by a matrix $A \in \operatorname{Sp}(n+1,1)$;

$$
\tilde{f}\left(\begin{array}{c}
z_{1} \\
\vdots \\
z_{n+2}
\end{array}\right)=A\left(\begin{array}{c}
z_{1} \\
\vdots \\
z_{n+2}
\end{array}\right)
$$

For brevity, write the form $\tilde{\theta}$ as follows:

$$
\tilde{\theta}=\bar{z}_{1}^{-1}\left(\left(\bar{z}_{1}, \ldots, \bar{z}_{n+2}\right) \cdot \mathrm{I}_{1, n+1} \cdot\left(\begin{array}{c}
d z_{1} \\
\vdots \\
d z_{n+2}
\end{array}\right)\right) z_{1}^{-1}
$$

Here

$$
I_{1, n+1}=\left(\begin{array}{cccc}
-1 & 0 & \cdots & 0 \\
0 & +1 & \cdots & 0 \\
\vdots & \vdots & \ddots & \vdots \\
0 & 0 & \cdots & +1
\end{array}\right)
$$

Then

$$
\tilde{f}^{*} \tilde{\theta}=\tilde{f}^{*}\left(\bar{z}_{1}^{-1}\right)\left(\left(\bar{z}_{1}, \ldots, \bar{z}_{n+2}\right) A^{*} \cdot \mathrm{I}_{1, n+1} \cdot A\left(\begin{array}{c}
d z_{1} \\
\vdots \\
d z_{n+2}
\end{array}\right)\right) \tilde{f}^{*}\left(z_{1}^{-1}\right)
$$


If $\chi^{\prime}: V_{0}^{4 n+7} \rightarrow \mathbb{F}$ is a smooth map defined by $\chi^{\prime}(z)=z_{1} \cdot \tilde{f}^{*} z_{1}^{-1}$ for $z=\left(z_{1}, \ldots, z_{n+2}\right)$, then by the definition

$$
\begin{aligned}
\tilde{f}^{*} \tilde{\theta} & =\left(\tilde{f}^{*} \bar{z}_{1}^{-1} \cdot \bar{z}_{1}\right)\left(\bar{z}_{1}^{-1} \cdot\left(\bar{z}_{1}, \ldots, \bar{z}_{n+2}\right) \cdot \mathrm{I}_{1, n+1} \cdot\left(\begin{array}{c}
d z_{1} \\
\vdots \\
d z_{n+2}
\end{array}\right) \cdot z_{1}^{-1}\right)\left(z_{1} \cdot \tilde{f}^{*} z_{1}^{-1}\right) \\
& =\bar{\chi}^{\prime} \cdot \tilde{\theta} \cdot \chi^{\prime} .
\end{aligned}
$$

On the other hand, for $t \in \mathbb{F}^{*}$,

$$
\begin{aligned}
\chi^{\prime}(z \cdot t) & =z_{1}(z \cdot t) \cdot z_{1}^{-1}(\tilde{f}(z \cdot t))=\left(z_{1} \cdot t\right) \cdot z_{1}(\tilde{f}(z) \cdot t)^{-1} \\
& =\left(z_{1} \cdot t\right) \cdot t^{-1} z_{1}(\tilde{f}(z))^{-1}=z_{1} \cdot\left(\tilde{f}^{*} z_{1}(z)\right)^{-1}=\chi^{\prime}(z) .
\end{aligned}
$$

Thus, $\chi^{\prime}$ factors through a map $\chi: S^{4 n+3} \rightarrow \mathbb{F}$ such that $\chi \circ \pi=\chi^{\prime}$. As $f \circ \pi=\pi \circ \tilde{f}$ and $\pi^{*} \theta=\tilde{\theta}$, we obtain that

$$
f^{*} \omega=\bar{\chi} \cdot \omega \cdot \chi
$$

By the definition, $f \in \operatorname{Aut}_{\mathrm{QCC}}\left(S^{4 n+3}\right)$.

(ii) Choose $\tilde{g} \in \operatorname{PSp}(n+1,1)_{\infty}$ such that $\lambda=a, \mu=b, B=I, x=y=z=0$ from (1.2), then $\tilde{g}$ has the form with respect to the basis $\left\{e_{1}, \ldots, e_{n+2}\right\}$ :

$$
\tilde{g}\left(\begin{array}{c}
z_{1} \\
z_{2} \\
\vdots \\
z_{n+1} \\
z_{n+2}
\end{array}\right)=\left(\begin{array}{c}
(a+b) z_{1}+(a-b) z_{n+2} / 2 \\
z_{2} \\
\vdots \\
z_{n+1} \\
(a-b) z_{1}+(a+b) z_{n+2} / 2
\end{array}\right) .
$$

On the other hand, $g^{*} \omega=\bar{\chi} \cdot \omega \cdot \chi$ from (i) where $\chi(\pi(z))=\chi^{\prime}(z)=z_{1} \cdot z_{1}(\tilde{g})^{-1}=$ $2 z_{1}\left((a+b) z_{1}+(a-b) z_{n+2}\right)^{-1}$. As $\chi(\infty)=\chi^{\prime}\left(f_{1}\right)=\chi^{\prime}\left(\frac{1}{\sqrt{2}}, 0, \ldots, 0, \frac{1}{\sqrt{2}}\right)$, we have $\chi(\infty)=a^{-1}$.

Proposition 12. $\operatorname{Aut}_{\mathrm{QCC}}\left(S^{4 n+3}\right)=\operatorname{PSp}(n+1,1)$. That is, the spherical pseudo-quaternionic geometry $\left(\mathrm{PSp}(n+1,1), S^{4 n+3}\right)$ coincides with the quaternionic Carnot-Carathéodory geometry $\left(\operatorname{Aut}_{\mathrm{QCC}}\left(S^{4 n+3}\right), S^{4 n+3}\right)$.

Proof. Put $H=\operatorname{Aut}_{\mathrm{QCC}}\left(S^{4 n+3}\right)$. Without of loss of generality, $H$ is assumed to be connected. We examine the structure of the Lie group $H$. Let $f$ be a diffeomorphism in $H$. By the definition $f^{*} \omega=\bar{\lambda} \cdot \omega \cdot \lambda$ where $\lambda: S^{4 n+3} \rightarrow \mathbb{F}^{*}$. First if the map $\mathrm{Sp}(1) \longrightarrow \operatorname{Aut}(\mathbb{F})=\mathrm{SO}(3)$ sends $\lambda /|\lambda|$ to $A$, then $f_{*}\left(\xi_{1}, \xi_{2}, \xi_{3}\right)=|\lambda|^{2}\left(\xi_{1}, \xi_{2}, \xi_{3}\right) \cdot A$ $(\bmod B)$. (See Definition 9.) Let $H_{\infty}$ be the stabilizer of $H$ at $\{\infty\}$, which contains $\operatorname{PSp}(n+1,1)_{\infty}$. Consider the tangential representation at $\{\infty\}$, $\tau: H_{\infty} \rightarrow \operatorname{Aut}\left(\mathrm{T}_{\{\infty\}} S^{4 n+3}\right)$.

Given $h \in H_{\infty}$ with $h^{*} \omega=\bar{\mu} \cdot \omega \cdot \mu$, suppose that $\mu(\infty)=1$. Then $h_{*}\left(\xi_{1}, \xi_{2}, \xi_{3}\right)=$ $\left(\xi_{1}, \xi_{2}, \xi_{3}\right)\left(\bmod B_{\infty}\right)$ at the point $\{\infty\}$. Recall the real valued four-form $\Omega=d \omega \wedge d \omega$ (cf. (1) in the proof of Lemma 8). Since $h^{*} d \omega=\bar{\mu} \cdot d \omega \cdot \mu(\bmod \omega)$, we have $h^{*} \Omega=$ $|\mu|^{4} \Omega(\bmod \omega)$. Put $h_{*}^{\prime}=h_{*} \mid B_{\infty}$. Since $h_{*} \operatorname{maps} B$ onto itself, $h_{*}^{\prime} \in \operatorname{Aut}\left(B_{\infty}\right)=$ $\mathrm{GL}(4 n, \mathbb{R})$. In particular, $h^{\prime *} \Omega_{\infty}=\Omega_{\infty}$ on $B_{\infty}$. Using the consisting relation (2) for $\Omega_{\infty}$ in the proof of Lemma 8 , the above formula implies that $h_{*}^{\prime} \in \operatorname{Sp}(n) \cdot \operatorname{Sp}(1)$. 
(Compare Lemma 9.1 [38] for example.) Thus, with respect to the basis $\omega^{\alpha}(\alpha=$ $1, \ldots, n)$ in (II) of Lemma 8 , there exist $\left(U_{\gamma}^{\alpha}\right) \in \operatorname{Sp}(n)$ and $b \in \operatorname{Sp}(1)$ such that $h^{\prime *} \omega^{\alpha}=U_{\gamma}^{\alpha} \cdot \omega^{\gamma} \cdot b$. Since $d \omega=-\frac{1}{2} \delta_{\bar{\alpha} \beta} \omega^{\bar{\alpha}} \wedge \omega^{3}(\bmod \omega)$ by (II) of Lemma 8, we have that $h^{\prime *} d \omega=\bar{b} \cdot d \omega \cdot b(\bmod \omega)$, which implies $b=\mu(\infty)=1$. Hence,

$$
\tau(h)=\left(\begin{array}{cc}
I_{3} & V \\
0 & U
\end{array}\right)
$$

with respect to the basis $\left\{\xi_{1}, \xi_{2}, \xi_{3}\right\}$ and that of $B_{\infty}$. Here $V$ is a $(3,4 n)$-matrix and $U=\left(U_{\gamma}^{\alpha}\right)$. If we denote by $\mathrm{M}(3,4 n)$ the vector space consisting of $(3,4 n)$-matrices, then $\tau(h) \in \mathrm{M}(3,4 n) \rtimes \mathrm{Sp}(n)$.

Now suppose that $f \in H_{\infty}$ and $f^{*} \omega=\bar{\lambda} \cdot \omega \cdot \lambda$. Put $\lambda(\infty)=a \in \mathbb{F}^{*}$. From Lemma 11 , choose $g \in \operatorname{Sp}(1) \times \mathbb{R}^{+}$such that $g^{*} \omega=\bar{\chi} \cdot \omega \cdot \chi$ with $\chi(\infty)=a^{-1}$. Consider the element $f \circ g \in H_{\infty}$. Since $(f \circ g)^{*} \omega=\overline{\chi \cdot g^{*} \lambda} \cdot \omega \cdot \chi \cdot g^{*} \lambda(\bmod \omega)$, we have that $\chi \cdot g^{*} \lambda(\infty)=\chi(\infty) \cdot \lambda(g(\infty))=1$. Then by the above argument and $\tau(g) \in \operatorname{Sp}(1) \times \mathbb{R}^{+}$, we conclude that

$$
\tau\left(H_{\infty}\right) \subset \mathrm{M}(3,4 n) \rtimes\left(\mathrm{Sp}(n) \cdot \mathrm{Sp}(1) \times \mathbb{R}^{+}\right) .
$$

In particular, $\operatorname{Sp}(n) \cdot \operatorname{Sp}(1)$ is a maximal compact subgroup of $\tau\left(H_{\infty}\right)$. If we note that $\tau$ maps compact groups of $H_{\infty}$ monomorphically into its image, the maximal compact subgroup of $H_{\infty}$ is $\operatorname{Sp}(n) \cdot \operatorname{Sp}(1)$ as well as $\operatorname{PSp}(n+1,1)_{\infty}$.

Let $K$ be a maximal compact subgroup of $H$. Since $\operatorname{Sp}(n+1) \cdot \operatorname{Sp}(1)$ is the maximal compact subgroup of $\operatorname{PSp}(n+1,1)$, we have that

$$
\operatorname{Sp}(n) \cdot \operatorname{Sp}(1) \subset \operatorname{Sp}(n+1) \cdot \operatorname{Sp}(1) \subset K .
$$

As $\operatorname{PSp}(n+1,1)$ acts transitively on the simply connected space $S^{4 n+3}$, we have $H / H_{\infty}=S^{4 n+3}$. In particular, $H_{\infty}$ is connected. By the structure theorem of connected Lie groups (cf. [15]), the coset space $H / K$ (resp. $H_{\infty} / \operatorname{Sp}(n) \cdot \operatorname{Sp}(1)$ ) is diffeomorphic to the euclidean space $\mathbb{R}^{m}$ (resp. $\mathbb{R}^{\ell}$ ) for some $m, \ell$. If we note that $\operatorname{Sp}(n) \cdot \operatorname{Sp}(1)=H_{\infty} \cap K$, then there is the fibration:

$$
K / \mathrm{Sp}(n) \cdot \mathrm{Sp}(1) \rightarrow H / H_{\infty} \longrightarrow \mathbb{R}^{m} / \mathbb{R}^{\ell} .
$$

Hence $K / \operatorname{Sp}(n) \cdot \operatorname{Sp}(1)=S^{4 n+3}$ and $m=\ell$. We obtain that $K=\operatorname{Sp}(n+1) \cdot \operatorname{Sp}(1)$.

Let $R \cdot S$ be the decomposition of $H$ where $R$ is the radical, and $S$ is a semisimple Lie group. If $\pi: H \longrightarrow \hat{S}$ is the canonical projection onto the semisimple Lie group $\hat{S}$ without center, then $\pi$ maps $\operatorname{PSp}(n+1,1)$ isomorphically onto the simple Lie subgroup $\pi(\operatorname{PSp}(n+1,1))$ of $\hat{S}$. Since $\pi(K)$ is a maximal compact subgroup of $\hat{S}$ and $K \subset \operatorname{PSp}(n+1,1)$, we have $\pi(\operatorname{PSp}(n+1,1))=\hat{S}$. Therefore $H$ is the semidirect product $R \rtimes \operatorname{PSp}(n+1,1)$.

On the other hand, $\pi\left(H_{\infty}\right)$ is a connected subgroup of $\pi(\operatorname{PSp}(n+1,1))$ containing $\pi\left(\operatorname{PSp}(n+1,1)_{\infty}\right)$. The classification theorem 4.4.1 of [8] implies that $\pi\left(H_{\infty}\right)=$ $\pi\left(\operatorname{PSp}(n+1,1)_{\infty}\right)$. Putting $R^{\prime}=R \cap H_{\infty}$, similarly we have that $H_{\infty}=$ $R^{\prime} \rtimes \operatorname{PSp}(n+1,1)_{\infty}$. Then

$$
S^{4 n+3}=H / H_{\infty}=R / R^{\prime} \times \operatorname{PSp}(n+1,1) / \operatorname{PSp}(n+1,1)_{\infty}=R / R^{i} \times S^{4 n+3} .
$$

Therefore $R=R^{\prime}$. In particular, it follows that $R=R_{\infty}$. As $H=R \rtimes \operatorname{PSp}(n+1,1)$ acts effectively and transitively on $S^{4 n+3}$, this implies that $R=\{1\}$. Hence $H=$ $\operatorname{PSp}(n+1,1)$. This completes the proof. 
For $f \in \operatorname{Aut}_{Q C C}\left(S^{4 n+3}\right)$, if $f^{*} \omega=\bar{\lambda} \cdot \omega \cdot \lambda$ satisfies that $|\lambda|=1$, i.e., $\lambda \in \operatorname{Sp}(1)$, then $f$ is said to be a strict quaternionic Carnot-Carathéodory transformation.

Corollary 13. If $\operatorname{Aut}_{\mathrm{QCC}}^{1}\left(S^{4 n+3}\right)$ is the group of strict quaternionic CarnotCarathéodory transformations of $S^{4 n+3}$, then the geometry ( $\operatorname{Aut}_{\mathrm{QCC}}^{1}\left(S^{4 n+3}\right), S^{4 n+3}$ ) coincides with the canonical Sasakian 3 -structured geometry $\left(\operatorname{Sp}(n+1) \cdot \operatorname{Sp}(1), S^{4 n+3}\right)$.

Proof. Let $\omega$ be the connection form on $S^{4 n+3}$ as before. Then $\omega=\omega_{1} i+\omega_{2} j+\omega_{3} k$ where $\omega_{i}$ is a real valued 1 -form. There is an $I=I_{1}, J=I_{2}, K=I_{3}$-invariant Riemannian metric on $S^{4 n+3}$ defined by

$$
g(X, Y)=\sum_{i=1}^{3} \omega_{i}(X) \cdot \omega_{i}(Y)+\frac{1}{3} \sum_{i=1}^{3} d \omega_{i}\left(X, I_{i} Y\right)
$$

Every element of $\operatorname{Aut}_{\mathrm{QCC}}^{1}\left(S^{4 n+3}\right)$ preserves $g$ (i.e., a compact group). It is noted that $g$ is the spherical metric on $S^{4 n+3}$.

Proposition 14. A spherical pseudo-quaternionic $(4 n+3)$-manifold $M$ admits a quaternionic Carnot-Carathéodory structure.

Proof. Let $B$ be the canonical quaternionic Carnot-Carathéodory structure on $S^{4 n+3}$ where $B=$ Null $\omega$. Given a maximal collection of charts $\left\{U_{\alpha}, \phi_{\alpha}\right\}_{\alpha \in \Lambda}$ of $M$ (cf. (1.1)), for each chart $\phi_{\alpha}: U_{\alpha} \rightarrow S^{4 n+3}$, we put

$$
B_{\alpha}=\phi_{\alpha}^{*} B, \omega_{\alpha}=\phi_{\alpha}^{*} \omega
$$

on $U_{\alpha}$. (Note that $B_{\alpha}=$ Null $\omega_{\alpha}$.) If $U_{\alpha} \cap U_{\beta} \neq \emptyset$, then $g_{\alpha \beta} \circ \phi_{\alpha}=\phi_{\beta}$ for an element $g_{\alpha \beta} \in \operatorname{PSp}(n+1,1)$. Since $g_{\alpha \beta}^{*} B=B$ by Proposition $12, B_{\alpha}=\phi_{\alpha}^{*} B=\phi_{\beta}^{*} B=B_{\beta}$ on $U_{\alpha} \cap U_{\beta}$. The union $\left\{B_{\alpha}\right\} \alpha \in \Lambda$ gives rise to a codimension 3 -subbundle $B^{\prime}$ on $M$. As $B^{\prime} \mid U_{\alpha}=B_{\alpha}$ is locally equivalent to $B$ (that is, each $\omega_{\alpha}$ satisfies (I), (II) of Lemma $8), B^{\prime}$ is a quaternionic Carnot-Carathéodory structure on $M$.

REMARK 15. (1) On $S^{4 n+3}$, we have obtained a globally defined $\mathfrak{s p}(1)$-valued oneform $\omega$ defining $B$ and three independent vector fields $\left\{\xi_{1}, \xi_{2}, \xi_{3}\right\}$ (equivalently, there exists the quaternionic structure of complex structures $\{I, J, K\}$ on $B$ ). In general, a spherical pseudo-quaternionic manifold $M$ admits a family of $\mathfrak{s p}(1)$-valued oneforms $\omega_{\alpha}$ and three independent vector fields $\left\{\xi_{1}^{\alpha}, \xi_{2}^{\alpha}, \xi_{3}^{\alpha}\right\}$ locally defined on each $U_{\alpha}$ (equivalently, a quaternionic structure $\left\{I_{\alpha}, J_{\alpha}, K_{\alpha}\right\}$ on each $B_{\alpha}$ ). If $U_{\alpha} \cap U_{\beta} \neq \emptyset$, then $g_{\alpha \beta} \circ \phi_{\alpha}=\phi_{\beta}$ with $g_{\alpha \beta} \in \operatorname{Aut}_{Q C C}\left(S^{4 n+3}\right)$, and so $\left(g_{\alpha \beta}\right)_{*}\left(\xi_{1}, \xi_{2}, \xi_{3}\right)=u^{2}\left(\xi_{1}, \xi_{2}, \xi_{3}\right) \cdot A$ for some $A: U_{\alpha} \cap U_{\beta} \rightarrow \mathrm{SO}(3)$. As $\left(\phi_{\alpha}\right)_{*}\left(\xi_{1}^{\alpha}, \xi_{2}^{\alpha}, \xi_{3}^{\alpha}\right)=\left(\xi_{1}, \xi_{2}, \xi_{3}\right)$, we have

$$
\left(\xi_{1}^{\beta}, \xi_{2}^{\beta}, \xi_{3}^{\beta}\right)=u^{-2}\left(\xi_{1}^{\alpha}, \xi_{2}^{\alpha}, \xi_{3}^{\alpha}\right) \cdot A^{-1}
$$

on $U_{\alpha} \cap U_{\beta}$. So the union $E=\left\{\xi_{1}^{\alpha}, \xi_{2}^{\alpha}, \xi_{3}^{\alpha}\right\}_{\alpha \in \Lambda}$ defines an $\mathrm{SO}(3) \times \mathbb{R}^{+}$-bundle over $M$.

(2) A (strict) quaternionic Carnot-Carathéodory transformation on a spherical pseudo-quaternionic $(4 n+3)$-manifold is similarly defined to be a diffeomorphism satisfying the condition of Definition 9 , that is, $f^{*} \omega_{\beta}=\bar{\lambda} \cdot \omega_{\alpha} \cdot \lambda$ whenever $f\left(U_{\alpha}\right) \subset U_{\beta}$.

Recall that $\operatorname{dim} \operatorname{Sp}(1) \cdot \operatorname{Sp}(n)=2 n^{2}+n+3$. By using the $G$-structure theory, we can prove the following: Let $M$ be a spherical pseudo-quaternionic $(4 n+3)$-manifold. Then the group of strict transformations $\operatorname{Aut}_{\mathrm{QCC}}^{1}(M)$ is a Lie group whose dimension is less than or equal to $2 n^{2}+5 n+6$. If $M$ is compact, then $\operatorname{Aut}_{\mathrm{QCC}}^{1}(M)$ is compact. 
A vector field $\xi$ is said to be a quaternionic Carnot-Carathéodory vector field if $\xi$ generates a local one-parameter group $\left\{\phi_{t}\right\}_{|t|<\varepsilon}$ of quaternionic Carnot-Carathéodory transformations of $M$. That is, for each $t, \phi_{t}$ is a quaternionic Carnot-Carathéodory transformation on a neighborhood.

Proposition 16 (cf. [20]). Let $B^{\prime}=\cup_{\alpha} B_{\alpha}$ be an (induced) Carnot-Carathéodory structure on a spherical pseudo-quaternionic manifold $M^{4 n+3}$. If $\xi$ is a nonzero quaternionic Carnot-Carathéodory vector field, then the set $\left\{x \in M \mid \xi_{x} \in B_{x}\right\}$ is a codimension 3-regular submanifold of $M$.

Proof. Put $\mathcal{N}=\left\{x \in M \mid \xi_{x} \in B_{x}^{\prime}\right\}$. Each $\mathfrak{s p}(1)$-valued one-form $\omega_{\alpha}$ on $U_{\alpha}$ can be described as

$$
\omega_{\alpha}=\omega_{\alpha}^{1} i+\omega_{\alpha}^{2} j+\omega_{\alpha}^{3} k
$$

such that $\phi_{\alpha}^{*} \omega_{i}=\omega_{\alpha}^{i}(i=1,2,3)$. Define a smooth map $f_{\alpha}: U_{\alpha} \rightarrow \mathbb{R}^{3}$ to be

$$
f_{\alpha}(p)=\left(\omega_{\alpha}\right)_{p}\left(\xi_{p}\right)=\left(\left(\omega_{\alpha}^{1}\right)_{p}\left(\xi_{p}\right),\left(\omega_{\alpha}^{2}\right)_{p}\left(\xi_{p}\right),\left(\omega_{\alpha}^{3}\right)_{p}\left(\xi_{p}\right)\right) .
$$

As $B_{\alpha}=B^{\prime} \mid U_{\alpha}=$ Null $\omega_{\alpha}, \mathcal{N} \cap U_{\alpha}=f_{\alpha}^{-1}(0)$. It is sufficient to show that $\operatorname{Rank}\left(d f_{\alpha}\right)_{p}=3$ for all $p \in \mathcal{N} \cap U_{\alpha}$. For this, let $\iota_{\xi}: A^{\ell}(M) \rightarrow A^{\ell-1}(M)$ be the interior product for each integer $\ell$ (cf. [25] for example). Then we have that $\iota_{\xi} \omega_{\alpha}(p)=\left(\omega_{\alpha}\right)_{p}\left(\xi_{p}\right)=f_{\alpha}(p)$. Since $L_{\xi} \omega_{\alpha}=\iota_{\xi} \cdot d \omega_{\alpha}+d \cdot \iota_{\xi} \omega_{\alpha}$, it follows that $d f_{\alpha}=L_{\xi} \omega_{\alpha}-\iota_{\xi} d \omega_{\alpha}: \mathrm{T} U_{\alpha} \rightarrow \mathrm{T} \mathbb{R}^{3}$. Let $\left\{\psi_{t}\right\}_{|t|<\varepsilon}$ be a local one-parameter group generated by $\xi$. By the definition, $\left(\psi_{t}\right)_{*}\left(B_{\alpha}\right)=B_{\alpha}$ for sufficiently small $t$. If $Y_{p} \in\left(B_{\alpha}\right)_{p}$, then

$$
\left(L_{\xi}\right)_{p} \omega_{\alpha}\left(Y_{p}\right)=\lim _{t \rightarrow 0} \frac{\left(\omega_{\alpha}\right)_{p}\left(Y_{p}\right)-\left(\omega_{\alpha}\right)_{\psi_{t}(p)}\left(\left(\psi_{t}\right)_{*}\left(Y_{p}\right)\right)}{t}=0 .
$$

It implies that $\left(d f_{\alpha}\right)_{p}(v)=-\left(\iota_{\xi} \cdot d \omega_{\alpha}\right)_{p}(v)=-2 d \omega_{\alpha}(\xi, v)$ for $v \in\left(B_{\alpha}\right)_{p}$. On the other hand, we have a family of local quaternionic structures $\left\{I_{\alpha}, J_{\alpha}, K_{\alpha}\right\}_{\alpha \in \Lambda}$ defined by the following commutative diagram:

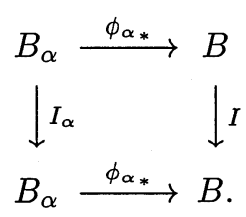

Similarly for $J_{\alpha}, K_{\alpha}$. Put

$$
v_{1}=-I_{\alpha} \xi / b, \quad v_{2}=-J_{\alpha} \xi / b, \quad v_{3}=-K_{\alpha} \xi / b
$$

where $b_{p}=g\left(\phi_{\alpha_{*}}\left(\xi_{p}\right), \phi_{\alpha_{*}}\left(\xi_{p}\right)\right)$. Then $v_{1}, v_{2}, v_{3}$ belong to $\left(B_{\alpha}\right)_{p}$ such that

$$
\begin{aligned}
d \omega_{\alpha}\left(\xi, v_{1}\right) & =(1,0,0), \\
d \omega_{\alpha}\left(\xi, v_{2}\right) & =(0,1,0), \\
d \omega_{\alpha}\left(\xi, v_{3}\right) & =(0,0,1) .
\end{aligned}
$$

In fact, as $\phi_{\alpha_{*}} \circ I_{\alpha}=I \circ \phi_{\alpha_{*}}$ by the definition,

$$
d \omega_{\alpha}\left(\xi, v_{1}\right)=d \phi_{\alpha}^{*} \omega\left(\xi, v_{1}\right)=d \omega\left(\phi_{\alpha_{*}} \xi,-I \phi_{\alpha_{*}} \xi / b\right)
$$


On the other hand, from the property $(*)$ of $(2.1)$ and that $g \mid B^{\prime} \times B^{\prime}$ is invariant under $I, J, K$,

$$
\begin{aligned}
d \omega\left(\phi_{\alpha_{*}} \xi,-I \phi_{\alpha_{*}} \xi / b\right) & =\frac{1}{b}\left(g\left(\phi_{\alpha_{*}} \xi, \phi_{\alpha_{*}} \xi\right) i+g\left(\phi_{\alpha_{*}} \xi, K \phi_{\alpha_{*}} \xi\right) j+g\left(\phi_{\alpha_{*}} \xi,-J \phi_{\alpha_{*}} \xi\right) k\right) \\
& =\frac{1}{b} g\left(\phi_{\alpha_{*}} \xi, \phi_{\alpha_{*}} \xi\right) i=i
\end{aligned}
$$

similarly for $v_{2}, v_{3}$. Therefore $f_{\alpha}^{-1}(0)$ is a codimension 3-regular submanifold of $U_{\alpha}$. If $U_{\alpha} \cap U_{\beta} \neq \emptyset$, then $g_{\alpha \beta}=\phi_{\beta} \circ \phi_{\alpha}^{-1}$ satisfies that $g_{\alpha \beta}^{*} \omega=\bar{\lambda} \cdot \omega \cdot \lambda$ for some function $\lambda: U_{\alpha} \cap U_{\beta} \rightarrow \mathbb{F}^{*}$ by Proposition 12. Then,

$$
f_{\beta}(p)=\left(\omega_{\beta}\right)_{p}\left(\xi_{p}\right)=\overline{\phi_{\alpha}^{*} \lambda}(p) \cdot f_{\alpha}(p) \cdot \phi_{\alpha}^{*} \lambda(p) .
$$

So $f_{\alpha}^{-1}(0)=f_{\beta}^{-1}(0)$ on $U_{\alpha} \cap U_{\beta}$. Since $\mathcal{N}=\cup_{\alpha \in \Lambda} \mathcal{N} \cap U_{\alpha}=\cup_{\alpha \in \Lambda} f_{\alpha}^{-1}(0), \mathcal{N}$ is a codimension 3-regular submanifold of $M$.

\section{Amenable holonomy and Classification.}

3.1. Quaternionic Carnot-Carathéodory structure on Heisenberg manifolds. Let $(\operatorname{Sim}(\mathcal{M}), \mathcal{M})$ be the Heisenberg geometry. We study the quaternionic Carnot-Carathéodory structure $B$ with the quaternionic structure $\{I, J, K\}$ on $\mathcal{M}$. This structure is induced from the quaternionic Carnot-Carathéodory structure of $S^{4 n+3}$ restricted to $S^{4 n+3}-\{\infty\}$ by Proposition 14 . As before, there is the equivariant principal bundle:

$$
\mathbb{R}^{3} \rightarrow(\operatorname{Sim}(\mathcal{M}), \mathcal{M}) \stackrel{\nu}{\longrightarrow}\left(\operatorname{Sim}\left(\mathbb{F}^{n}\right), \mathbb{F}^{n}\right) .
$$

LEMMA 17.

(1) The fiber $\mathbb{R}^{3}$ is transverse to $B$.

(2) The center $\mathbb{R}^{3}$ is compatible with $\{I, J, K\}$. i.e., $t_{*} \circ I=I \circ t_{*}$ for all $t \in \mathbb{R}^{3}$, similarly for $J, K$.

Proof. (1) Let $\xi$ be a nontrivial vector field induced by a one-parameter subgroup of $\mathbb{R}^{3}$. So $\xi$ is a quaternionic Carnot-Carathéodory vector field. Suppose that $\xi_{p} \in B_{p}$ for some point $p \in \mathcal{M}$. Since $\mathbb{R}^{3}$ is the center of $\mathcal{M}, g_{*} \xi_{p}=\xi_{g p}$ for all $g \in \mathcal{M}$. In particular we have $\xi_{x} \neq 0$ for all $x \in \mathcal{M}$. As $B$ is invariant under the action of $\mathcal{M}$, the subspace $\left\{x \in \mathcal{M} \mid \xi_{x} \in B_{x}\right\}$ coincides with the whole space $\mathcal{M}$. This contradicts Proposition 16. Thus $\mathbb{R}^{3}$ is transversal to $B$ at each point of $\mathcal{M}$.

(2) Recall that $\mathcal{M}=S^{4 n+3}-\{\infty\}=\{[z, y, 1]\} \subset \mathrm{IPP}^{n+1}$ with respect to the basis $\left\{f_{1}, e_{2}, \ldots, e_{n+1}, f_{n+2}\right\}$ (cf. (1.2)). Let $U=\{[z, y, \mu] \mid \mu \neq 0\}$ be an open subset in $\mathbb{F P}^{n+1}$. Each $t \in \mathbb{R}^{3}$ satisfies that $t[z, y, \mu]=[z+t \mu, y, \mu]$. If $\varphi: \mathcal{M} \subset U \rightarrow \mathbb{F}^{n+1}$ is a parametrization defined by $\varphi([z, y, \mu])=\left(z \mu^{-1}, y \mu^{-1}\right)$, then the action of $\mathbb{R}^{3}$ on $\mathcal{M}$ is equivalent to the usual translations of $\mathbb{R}^{3}$ on $\mathbb{R}^{3} \times \mathbb{F}^{n} \subset \mathbb{F}^{n+1}$ :

$$
\varphi \cdot t \cdot \varphi^{-1}(w, x)=(w+t, x) .
$$

Chasing the commutative diagram from the definition of (2.3):

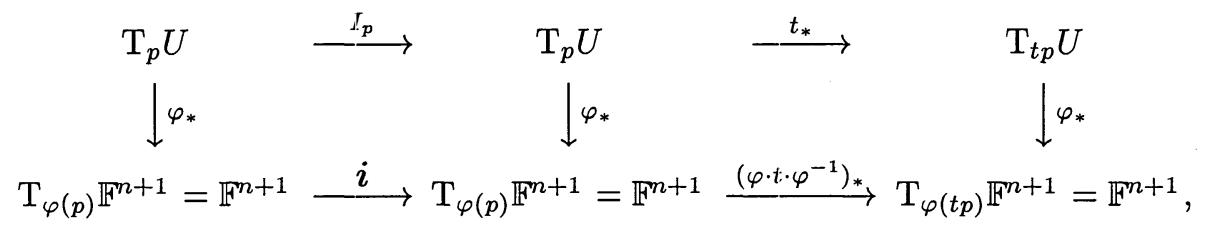


we obtain that $\left(\varphi \cdot t \cdot \varphi^{-1}\right)_{*}=$ id and hence $t_{*} \circ I=I \circ t_{*}$ for all $t \in \mathbb{R}^{3}$, similarly for $J, K$.

This lemma implies the following corollary.

Corollary 18. Let $(B,\{I, J, K\})$ be the induced quaternionic Carnot-Carathéodory structure on $\mathcal{M}$. Then $\nu$ induces the standard quaternionic structure $\left\{I_{0}, J_{0}, K_{0}\right\}$ on $\mathbb{F}^{n}$,i.e., $\nu_{*} \circ I=I_{0} \circ \nu_{*}$, etc. In particular, $\nu_{*}$ maps $(B,\{I, J, K\})$ isomorphically onto the tangent bundle $\left(\mathrm{TF}^{n},\left\{I_{0}, J_{0}, K_{0}\right\}\right)$ at each point of $\mathcal{M}$.

3.2. Dilations on Heisenberg manifolds. Choose a left invariant metric $g$ on $\mathcal{M}$ with the group of isometries $\mathrm{E}(\mathcal{M})=\mathcal{M} \rtimes \operatorname{Sp}(n) \cdot \operatorname{Sp}(1)$. If we note that $\nu$ is a homomorphism of $\mathcal{M}$ onto $\mathbb{F}^{n}$, then $g$ induces the standard euclidean metric $g_{0}$ on $\mathbb{F}^{n}$. Corollary 18 implies that $\nu_{*}:(B, g,\{I, J, K\}) \rightarrow\left(\mathbb{T F}^{n}, g_{0},\left\{I_{0}, J_{0}, K_{0}\right\}\right)$ is a local isometry at each point of $\mathcal{M}$. As $g_{0}$ is invariant under $\left\{I_{0}, J_{0}, K_{0}\right\}, g \mid B \times B$ is invariant under $\{I, J, K\}$. Let $\lambda: \operatorname{Sim}(\mathcal{M}) \longrightarrow \mathbb{R}^{+}$be the scale factor homomorphism as well as $\lambda_{0}: \operatorname{Sim}\left(\mathbb{F}^{n}\right)=\mathbb{F}^{n} \rtimes\left(\operatorname{Sp}(n) \cdot \operatorname{Sp}(1) \times \mathbb{R}^{+}\right) \longrightarrow \mathbb{R}^{+}$. Since $g_{0}$ satisfies that $\left(g_{0}\right)_{h p}\left(h_{*} X, h_{*} Y\right)=\lambda_{0}(h)^{2} \cdot\left(g_{0}\right)_{p}(X, Y)$ for each $h \in \operatorname{Sim}\left(\mathbb{F}^{n}\right)$, we have for each $\alpha \in \operatorname{Sim}(\mathcal{M})$, and $X, Y \in B_{x}$

$$
g_{\alpha x}\left(\alpha_{*} X, \alpha_{*} Y\right)=\lambda(\alpha)^{2} \cdot g_{x}(X, Y) .
$$

Therefore $(\operatorname{Sim}(\mathcal{M}), g \mid B \times B, \mathcal{M})$ plays the same role as the euclidean similarity geometry. The similar property holds for $\left(\operatorname{Sim}(\mathcal{M}), g \mid \mathrm{TR}^{3} \times \mathrm{TR}^{3}\right)$. In fact, if $h=$ $((\alpha, \beta, \gamma), z) \cdot(A \cdot g, t) \in \operatorname{Sim}(\mathcal{M})$, then for $w=(w, 0) \in \mathbb{R}^{3}$ and $x \in \mathcal{M}$,

$$
h(w \cdot x)=t^{2} \cdot g w g^{-1} \cdot h x .
$$

Since $\mathbb{R}^{3}$ is the normal subgroup of $\operatorname{Sim}(\mathcal{M})$, each element of $\operatorname{Sim}(\mathcal{M})$ leaves the subbundle $\mathbb{T R}^{3}$. Moreover, if $\left\{\xi_{1}, \xi_{2}, \xi_{3}\right\}$ are the vector fields which generate $\mathbb{R}^{3}$, then $h_{*}\left(\left(\xi_{i}\right)_{x}\right)=t^{2}\left(\operatorname{Ad}_{g} \xi\right)_{h x}=\lambda(h)^{2}\left(\operatorname{Ad}_{g} \xi\right)_{h x}$. As Ad acts as isometries with respect to $g$, we have that for $X, Y \in \mathrm{TR}_{x}^{3}$,

$$
g_{h x}\left(h_{*} X, h_{*} Y\right)=\lambda(h)^{4} \cdot g_{x}(X, Y) .
$$

Denote by $\mathcal{F}$ the frame bundle on $\mathcal{M}$ generated by $\left\{\xi_{1}, \xi_{2}, \xi_{3}\right\}$. Since $\mathcal{F}^{\perp}=B$ with respect to $g$ and $B$ is invariant under $\operatorname{Sim}(\mathcal{M})$, there is a $\operatorname{Sim}(\mathcal{M})$-invariant direct decomposition: $\mathrm{T} \mathcal{M}=\mathcal{F} \oplus B$, or equivalently $g=g|\mathcal{F} \times \mathcal{F} \oplus g| B \times B$.

3.3. Classification of compact manifolds with amenable holonomy. A representation $\rho: \pi \rightarrow \operatorname{PSp}(n+1,1)$ is said to be amenable if the closure of the image $\overline{\rho(\pi)}$ in $\operatorname{PSp}(n+1,1)$ lies in the maximal amenable Lie subgroup of $\operatorname{PSp}(n+1,1)$. As the first step to prove Theorem $\mathrm{C}$ of Introduction, we must show that

THEOREM 19. Let $M$ be a compact spherical pseudo-quaternionic $(4 n+3)$ manifold. If the holonomy group is amenable, then $M$ is finitely covered by the sphere $S^{4 n+3}$, a Hopf manifold $S^{1} \times S^{4 n+2}$ or a nilmanifold $\mathcal{M} / \Gamma$.

The rest of this section is spent for the proof of the above theorem. Suppose that a compact smooth connected $(4 n+3)$-manifold $M$ admits a spherical pseudoquaternionic structure. Then there exists a developing pair $(\rho, \operatorname{dev}):(\pi, \tilde{M}) \longrightarrow$ $\left(\operatorname{PSp}(n+1,1), S^{4 n+3}\right)$. A maximal amenable subgroup of $\operatorname{PSp}(n+1,1)$ is conjugate to $\operatorname{Sp}(n) \cdot \operatorname{Sp}(1)$ or $\operatorname{Sim}(\mathcal{M})$. If the holonomy group $\rho(\pi)$ is amenable, then we can assume that $\rho(\pi)$ lies in $\operatorname{Sp}(n) \cdot \operatorname{Sp}(1)$ or $\operatorname{Sim}(\mathcal{M})$. In the former case, choose a 
spherical metric on $S^{4 n+3}$ such that $\operatorname{Sp}(n) \cdot \operatorname{Sp}(1)$ is a subgroup of isometries. The pullback by the developing map gives a $\pi$-invariant Riemannian metric on $\tilde{M}$. This metric induces a Riemannian metric on $M$. As $M$ is compact by our hypothesis, $\tilde{M}$ is complete. Therefore dev is a local isometry of a cornplete Riemannian manifold $\tilde{M}$ into $S^{4 n+3}$. Hence dev is a covering map (cf. [7]). Thus dev is homeomorphic so that $M \approx S^{4 n+3} / F$ where $F=\rho(\pi) \subset \operatorname{Sp}(n) \cdot \operatorname{Sp}(1)$ is a finite subgroup acting freely on $S^{4 n+3}$.

In the latter case, $\rho(\pi)$ lies in $\operatorname{Sim}(\mathcal{M})$. There is no Riemannian metric invariant under $\operatorname{Sim}(\mathcal{M})$.

3.4. Complete similarity manifolds. Given a Heisenberg similarity manifold $N$, there exists a developing pair $(\rho, \mathrm{dev}):\left(\pi_{1}(N), \tilde{N}\right) \longrightarrow(\operatorname{Sim}(\mathcal{M}), \mathcal{M})$. In general, $N$ is said to be geodesically complete if the developing map is a homeomorphism of $\tilde{N}$ onto $\mathcal{M}$. Let $\nabla$ be a left invariant Levi-Civita connection on $\mathcal{M}$ induced by $g$. Since $\nabla$ is invariant under the automorphism group of $\mathcal{M}$, each element of $\operatorname{Sim}(\mathcal{M})$ preserves $\nabla$. Then the pullback connection $\nabla^{\prime}$ by dev defines a $\pi_{1}(N)$-invariant LeviCivita connection on $\tilde{N}$, since it is also induced from the pullback Riemannian metric $g^{\prime}=\operatorname{dev}^{*} g$. Thus $\nabla^{\prime}$ induces an affine connection on $N$. In other words, geodesically completeness on $N$ is equivalent to that the exponential map is defined on the entire tangent space $T_{x} \tilde{N}$ for some point $x \in \tilde{N}$ (cf. [7]). This does not depend on the choice of a point in $\tilde{N}$ because geodesically completeness on $\tilde{N}$ is the same as metric completeness by $g^{\prime}$. (See [46].) However, note that the Riemannian metric $g^{\prime}$ does not necessarily induce a Riemannian metric on $N$.

Put $\Gamma=\rho(\pi)$. Assume that $\Gamma$ is infinite and amenable in $\operatorname{Sim}(\mathcal{M})$. Put $\tilde{M}^{\prime}=$ $\tilde{M}-\operatorname{dev}^{-1}(\infty)$. Then the developing pair reduces to the following:

$$
(\rho, \mathrm{dev}):\left(\pi, \tilde{M}^{\prime}\right) \longrightarrow(\operatorname{Sim}(\mathcal{M}), \mathcal{M}) .
$$

Recall that $\mathcal{M}$ supports the Carnot-Carathéodory structure $B$ and the frame bundle $\mathcal{F}$.

As dev is an immersion, we have the pullback metric $g^{\prime}=\operatorname{dev}^{*} g$, the induced subbundles $B^{\prime}=\operatorname{dev}^{*} B$ and $\mathcal{F}^{\prime}=\operatorname{dev}^{*} \mathcal{F}$ on $\tilde{M}^{\prime}$ respectively. There exists a ball $D_{r}(x)$ about zero of radius $r$ in $\mathrm{T}_{x} \tilde{M}^{\prime}$ with respect to $g^{\prime}$ such that the exponential map $\exp _{x}: D_{r}(x) \rightarrow \tilde{M}^{\prime}$ is defined. Obviously there is the commutative diagram: $(\operatorname{dev}(x)=p)$

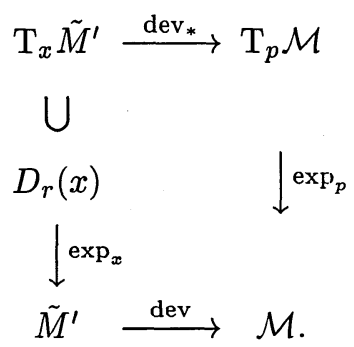

If $\tilde{M}^{\prime}$ is (geodesically) complete, the local isometry dev is a homeomorphism of $\tilde{M}^{\prime}$ onto $\mathcal{M}$. The holonomy group $\Gamma$ will be discrete in $\operatorname{Sim}(\mathcal{M})$. If $\operatorname{dev}^{-1}(\infty) \neq \emptyset$, then it is easy to see that dev $: \tilde{M} \longrightarrow S^{4 n+3}$ is homeomorphic. Since $\Gamma$ is infinite by our hypothesis, we have that $\tilde{M}=\tilde{M}^{\prime}$. Recall that $\mathbb{R}^{+} \subset \operatorname{Sim}(\mathcal{M})$ acts on $\mathcal{M}$ as expansion or contraction as in (1.2). If $\Gamma$ is discrete in $\operatorname{Sim}(\mathcal{M})$, then $\Gamma$ is either conjugate to a subgroup of $\mathcal{M} \rtimes \mathrm{Sp}(n) \cdot \mathrm{Sp}(1)=\mathrm{E}(\mathcal{M})$ or $\operatorname{Sp}(n) \cdot \operatorname{Sp}(1) \times \mathbb{R}^{+}$. As the 
latter group fixes the origin of $\mathcal{M}, \Gamma \subset \mathrm{E}(\mathcal{M})$ in our case. Hence $M$ is isomorphic to an infranilmanifold $\mathcal{M} / \Gamma$.

3.5. Incomplete similarity manifolds. Suppose that $\tilde{M}^{\prime}$ is incomplete. Define

$$
R(x)=\sup \left\{r \mid \exp _{x}: D_{r}(x) \rightarrow \tilde{M}^{\prime} \text { is defined }\right\} .
$$

Then $R(x)<\infty$ for all points $x$ of $\tilde{M}^{\prime}$ by the above remark (3.4). Put $r=R(x)$. With respect to the pull-back affine connection $\nabla^{\prime}$, there exists a vector $v \in \partial D_{r}(x)$ such that $\gamma(t)=\exp _{x} t v$ is an incomplete geodesic, i.e., $\gamma(t)$ is defined on $0 \leqq t<1$ but not $t=1$. Recall that $M=\tilde{M} / \pi$ decomposes into the union of the orbit space $\tilde{M}^{\prime} / \pi$ and $\operatorname{dev}^{-1}(\infty) / \pi$.

LEMMA 20. Let $P: \tilde{M} \rightarrow M$ be the covering map. Then the geodesic image $\{P(\gamma(t))\}_{0 \leqq t<1}$ has an accumulation point inside $\tilde{M}^{\prime} / \pi$.

Proof. Since $M$ is compact, the geodesic image $\{P(\gamma(t))\}_{0 \leqq t<1} \in \tilde{M}^{\prime} / \pi$ has an accumulation point $y$ in $M$. Since $\mathrm{dev}^{-1}(\infty) / \pi$ consists of finite points, we may consider the case that $\operatorname{dev}^{-1}(\infty) / \pi$ consists of a single point. Suppose that $\operatorname{dev}^{-1}(\infty) / \pi=\{y\}$ in $M$. Choose an evenly covered neighborhood $U$ of $y$. When $\tilde{U}$ is a lift of $U$ to $\tilde{M}$, by the definition, $\alpha \cdot \tilde{U} \cap \tilde{U} \neq \emptyset$ for some $\alpha \in \pi$ if and only if $\alpha=1$. We can assume that $\operatorname{dev}: \tilde{U} \rightarrow \operatorname{dev}(\tilde{U})$ is homeomorphic. If $\tilde{y} \in \tilde{U}$ is a point with $P(\tilde{y})=y$, then $\operatorname{dev}(\tilde{y})=\{\infty\}$ as above.

As $y$ is an accumulation point of the geodesic image $\{P(\gamma(t))\}_{0 \leqq t<1}$, there exists a sequence $0<t_{1}<t_{2}<\cdots<t_{n}<\cdots<1$ such that

$$
\gamma\left(t_{n}\right) \in \alpha_{n} \cdot \tilde{U}
$$

for elements $\alpha_{n} \in \pi$. On the other hand, as $\mathcal{M}$ is complete, there exists a limit point $\lim _{n \rightarrow \infty} \operatorname{dev}\left(\gamma\left(t_{n}\right)\right) \in \mathcal{M}$. Since $\operatorname{dev}\left(\gamma\left(t_{n}\right)\right) \in \rho\left(\alpha_{n}\right) \operatorname{dev}(\tilde{U})$ and each $\rho\left(\alpha_{n}\right)$ stabilizes the point $\{\infty\}$, we have that

$$
\lim _{n \rightarrow \infty} \operatorname{dev}\left(\gamma\left(t_{n}\right)\right)=\bigcap_{n>>1}^{\infty} \rho\left(\alpha_{n}\right) \operatorname{dev}(\tilde{U})=\{\infty\},
$$

which is a contradiction. Therefore the accumulation point $y$ of the geodesic image $\{P(\gamma(t))\}_{0 \leqq t<1}$ lies in $\tilde{M}^{\prime} / \pi$.

Then we can apply the same argument of Fried [12], also Miner [31] to the quaternionic case. By Lemma 20, the geodesic image in $\tilde{M}^{\prime} / \pi$ has an accumulation point $z$ inside $\tilde{M}^{\prime} / \pi$, and it passes by $z$ infinitely many times and arbitrarily close. By the argument of [12], [31], this recurrent property gives a family of elements $\left\{\gamma_{i j}\right\}$ of $\pi_{1}(M, z)$ such that $\gamma_{i j}(j>>i)$ maps $\gamma\left(t_{i}\right)$ very close to $\gamma\left(t_{j}\right)\left(0<t_{i}<t_{j}<1\right)$. Moreover

Lemma 21 ([12], Lemma $3.2[31])$. Denote by $0=(0,0)$ the origin of $\mathcal{M}$. Suppose that $\exp _{p} \circ \operatorname{dev}_{*}(v)=0 \in \mathcal{M}(\operatorname{dev}(x)=p)$. For sufficiently large $i, j$, the holonomy image $\rho\left(\gamma_{i j}\right)$ can be chosen to be a Heisenberg similarity transformation centered arbitrarily close to 0 with arbitrarily small rotation matrix.

With the aid of Lemma $21, \exp _{x}$ can be defined on the half space $H_{x}=\{X \in$ $\left.\mathrm{T}_{x} \tilde{M}^{\prime} \mid g_{x}^{\prime}(v, X)<r^{2}\right\}$ containing $D_{r}(x)$. (See the figures of [12], [31].) To see this, note that $\exp _{p} \operatorname{dev}_{*}\left(D_{r}(x)\right)$ is a maximal metric ball about $p$ of radius $r$ whose boundary contains 0 . Let $X \in H_{x}$. By Lemma 21, there exists an element $\rho\left(\gamma_{i j}\right) \in \Gamma$ such 
that for sufficiently large $i<j, \rho\left(\gamma_{i j}\right) \circ \exp _{p} \operatorname{dev}_{*}(X) \in \exp _{p} \operatorname{dev}_{*}\left(D_{r}(x)\right)$, and $\gamma_{i j} x \in$ $\exp _{x}\left(D_{r}(x)\right)$. Using $\operatorname{dev}^{-1}$ locally, we have that $\exp _{\gamma_{i j} x}\left(\left(\gamma_{i j}\right)_{*} X\right) \in \exp _{x}\left(D_{r}(x)\right)$. Define $\exp _{x} X=\gamma_{i j}{ }^{-1} \circ \exp _{\gamma_{i j} x}\left(\left(\gamma_{i j}\right)_{*} X\right)$.

Since $\operatorname{dev}\left(\gamma_{i j}{ }^{-1} \circ \exp _{\gamma_{i j} x}\left(\gamma_{i j}\right)_{*} X\right)=\exp _{p} \operatorname{dev}_{*}(X)$, which is well defined. As a consequence, for each $y \in \tilde{M}^{\prime}$, there exists a unique vector $V=V_{y} \in \partial D_{r}(y)$ for which $\exp _{y} V$ is not defined.

REMARK 22. From the property (3.2) of Heisenberg dilations, there is no $\pi$ invariant Riemannian metric within the conformal class on $\tilde{M}^{\prime}$, although it is possible to construct a $\pi$-invariant Riemannian metric within the Carnot-Carathéodory structure. For example, denote

$$
\begin{aligned}
& R^{b}(x)=\sup \left\{\left\|X^{b}\right\| ; X \in D_{r}(x), \exp _{x} X \text { is not defined }\right\} \\
& R^{f}(x)=\sup \left\{\left\|X^{f}\right\| ; X \in D_{r}(x), \exp _{x} X \text { is not defined }\right\} .
\end{aligned}
$$

Obviously, $0 \leqq R^{b}(x), R^{f}(x) \leqq R(x)$. Since the decomposition is invariant under $\pi$, the properties $(*),(* *)$ of (3.2) imply that for $\gamma \in \pi$,

$$
\begin{aligned}
g_{\gamma \cdot s ;}^{\prime}\left(\gamma_{*} X^{b}, \gamma_{*} Y^{b}\right) & =\lambda(\rho(\gamma))^{2} \cdot g^{\prime}{ }_{x}\left(X^{b}, Y^{b}\right), \\
g_{\gamma \cdot x}^{\prime}\left(\gamma_{*} X^{f}, \gamma_{*} Y^{f}\right) & =\lambda(\rho(\gamma))^{4} \cdot g^{\prime}{ }_{x}\left(X^{f}, Y^{f}\right) .
\end{aligned}
$$

Let $Y=Y^{f} \oplus Y^{b}$ for a vector $Y \in T_{x} \tilde{M}^{\prime}$. We introduce a new metric:

$$
\tilde{g}_{x}(X, Y)=\frac{g_{x}^{\prime}\left(X^{f}, Y^{f}\right)}{R^{f}(x)^{2}+R^{b}(x)^{4}}+\frac{g^{\prime}{ }_{x}\left(X^{b}, Y^{b}\right)}{R^{f}(x)+R^{b}(x)^{2}} .
$$

Then $\tilde{g}$ is a Riemannian metric on $\tilde{M}^{\prime}$ which is invariant under $\pi$.

For each $x \in \tilde{M}^{\prime}$, we have the half space $H_{x}=\left\{X \in \mathrm{T}_{x} \tilde{M}^{\prime} \mid g_{x}^{\prime}(V, X)<r^{2}\right\}$ on which $\exp _{x}$ is defined. Here $V=V_{x}$ is a unique vector lying on the boundary $\partial D_{r}(x)$ such that $\exp _{x} V$ is undefined, where $r=R(x)=\|V\|=\sqrt{\left\|V^{f}\right\|^{2}+\left\|V^{b}\right\|^{2}}$. Unfortunately, the half spaces $H_{x}$ are not necessarily translated each other by the elements of $\pi$.

Let $\partial H_{x}=\left\{X \in \mathrm{T}_{x} \tilde{M}^{\prime} \mid g_{x}^{\prime}(V, X)=r^{2}\right\}$ denote the boundary of $H_{x}$. The following lemma is obtained from the idea of Fried [12] (also Miner [31]) which has been already used to show the existence of half space $H_{x}$ on which $\exp _{x}$ is defined.

Lemma 23 (Compare Lemma 1 [12], Proposition 2.6 [31].). Let 0 be the origin of $\mathcal{M}$ and suppose $\exp _{p} \circ \operatorname{dev}_{*}(v)=0$ as above. Then

$$
0 \in \bigcap_{y \in \tilde{M}^{\prime}} \exp _{q} \circ \operatorname{dev}_{*}\left(\partial H_{y}\right), \quad \operatorname{dev}(y)=q .
$$

Proof. Suppose not. Then the origin 0 is not contained in $\exp _{q} \circ \operatorname{dev}_{*}\left(\partial H_{y}\right)$ for some $y \in \tilde{M}^{\prime}$. By Lemma 21, there is an element $\gamma \in \pi$ whose holonomy $\rho(\gamma)$ carries the half space $\exp _{q} \circ \operatorname{dev}_{*}\left(H_{y}\right)$ arbitrarily close to 0 in $\mathcal{M}$. For the vector $V=V_{y} \in \partial D_{R(y)}(y), \gamma_{*} V$ is a unique vector of $\partial D_{R(\gamma y)}(\gamma y)$ such that $\exp _{\gamma y} \gamma_{*} V$ is undefined. So the geodesic $\exp _{\rho(\gamma) q}\left(t \cdot \operatorname{dev}_{*}\left(\gamma_{*} V\right)\right)(0 \leqq t<1)$ lies in the half space $\exp _{\rho(\gamma) q} \circ \operatorname{dev}_{*} H_{\gamma y}$, and it meets the boundary $\exp _{\rho(\gamma) q}\left(\operatorname{dev}_{*}\left(\partial H_{\gamma y}\right)\right)$ perpendicularly at the point $\exp _{\rho(\gamma) q}\left(\operatorname{dev}_{*}\left(\gamma_{*} V\right)\right)$. Denote by $\prec_{y}(V, Y)$ the angle between $V$ and $Y$ at $y$ and let $D_{R(y)}^{\varepsilon}(y)=\left\{Y \in D_{R(y)}(y) \mid \prec_{y}(V, Y)<\varepsilon\right\}$ be the cone at $y$ of the axis 
$V$ with angle $\varepsilon>0$. As the geodesic $\exp _{\rho(\gamma) q}\left(t \cdot \operatorname{dev}_{*}\left(\gamma_{*} V\right)\right)$ is arbitrarily close to 0 , we choose a small $\varepsilon$ such that

$$
\exp _{q} \circ \operatorname{dev}_{*}\left(D_{R(y)}^{\varepsilon}(y)\right) \subset \exp _{\rho(\gamma) q} \circ \operatorname{dev}_{*}\left(H_{\gamma y}\right) .
$$

By the commutativity, $\operatorname{dev} \circ \exp _{y}\left(D_{R(y)}^{\varepsilon}(y)\right) \subset \operatorname{dev} \circ \exp _{\gamma y}\left(H_{\gamma y}\right)$, which implies that $\exp _{y}\left(D_{R(y)}^{\varepsilon}(y)\right)$ is properly embedded in the convex domain $\exp _{\gamma y}\left(H_{\gamma y}\right)$. The closed metric cone $\overline{\exp _{y}\left(D_{R(y)}^{\varepsilon}(y)\right)}$ sits inside $\exp _{\gamma y}\left(H_{\gamma y}\right)$ and hence is compact. This contradicts that $\exp _{y} V$ is undefined.

Put $J=\bigcap_{y \in \tilde{M}^{\prime}} \exp _{q} \circ \operatorname{dev}_{*}\left(\partial H_{y}\right)$. By the construction of $J$, the developing image $\operatorname{dev}\left(\tilde{M}^{\prime}\right)$ is obviously outside $J$.

Corollary 24. $\operatorname{dev}\left(\tilde{M}^{\prime}\right) \subset \mathcal{M}-J$.

Lemma 25. Let $\bar{J}$ be the closure of $J$ in $S^{4 n+3}$ Then, either $\bar{J}=J$ or $\bar{J}=J \cup\{\infty\}$.

Proof. Since $S^{4 n+3}=\mathcal{M} \cup\{\infty\}$, and $J \subset \exp _{q} \circ \operatorname{dev}_{*}\left(\partial H_{y}\right)$, it is easy to see that $\bar{J} \subset J \cup\{\infty\}$ and so either $\bar{J}=J$ or $\bar{J}=J \cup\{\infty\}$.

LEMMA 26. If $\bar{J}=J \cup\{\infty\}$, then $\mathrm{dev}^{-1}(\infty)=\emptyset$. As a consequence, the developing map reduces to the following:

$$
\operatorname{dev}: \tilde{M} \longrightarrow \mathcal{M}-J
$$

Proof. If $x \in \mathrm{dev}^{-1}(\infty)$, then there is a neighborhood $U$ of $x$ in $\tilde{M}$ with $U-\{x\} \subset$ $\tilde{M}^{\prime}$ such that $\operatorname{dev}(U-\{x\})=\operatorname{dev}(U)-\{\infty\} . \operatorname{As}\{\infty\} \in \bar{J}-J,(\operatorname{dev}(U)-\{\infty\}) \cap J \neq \emptyset$, but $\operatorname{dev}(U)-\{\infty\} \subset \operatorname{dev}\left(\tilde{M}^{\prime}\right)$, which is impossible by Corollary 24. In particular, $\tilde{M}=\tilde{M}^{\prime}$.

As above, the unique vector $V \in \partial D_{r}(x)$ has the property that $\exp _{x}(t \cdot V)$ is defined for $0 \leqq t<1$, but not $t=1$. In this case, the image $\exp _{p}\left(\operatorname{dev}_{*} V\right)(\operatorname{dev}(x)=p)$ is said to be an invisible point. In general if $\exp _{x}(t \cdot X)$ is defined for $t=1$, then $\exp _{p}\left(\operatorname{dev}_{*} X\right)$ is called a visible point because $\exp _{p}\left(\operatorname{dev}_{*} X\right)=\operatorname{dev}$ oxp $\exp _{x}(X)$, otherwise the point $\exp _{p}\left(\operatorname{dev}_{*} X\right)$ is invisible. Especially every point in the half space $\exp _{q}\left(\operatorname{dev}_{*} H_{y}\right)$ is visible for each $y \in \tilde{M}^{\prime}$, while invisible points lie only on the boundary $\exp _{q} \circ \operatorname{dev}_{*}\left(\partial H_{y}\right)$. (Compare [12], [31].)

LEMMA 27. $J$ is invariant under $\Gamma$. In particular, $\bar{J}$ is a $\Gamma$-invariant closed subset in $S^{4 n+3}$ contained in a submanifold of dimension at most $4 n+2$.

Proof. Let $m \in J$. There exists a vector $X \in \partial H_{y}$ such that $m=\exp _{q}\left(\operatorname{dev}_{*}(X)\right)$ for each $y \in \tilde{M}^{\prime}(\operatorname{dev}(y)=q)$. If we note that each boundary $\exp _{q} \circ \operatorname{dev}_{*}\left(\partial H_{y}\right)$ contains 0 from Lemma 23 , then $\exp _{q}\left(\operatorname{dev}_{*} X\right)$ is an invisible point, otherwise $\exp _{q}\left(\operatorname{dev}_{*}(V)\right)$ would be a visible point. Since each $\gamma \in \pi$ maps the unique vector $V \in \partial D_{R(y)}(y)$ onto the unique vector $\gamma_{*} V \in \partial D_{R(\gamma y)}(\gamma y)$, the geodesic $\exp _{\rho(\gamma) q}\left(t \cdot \operatorname{dev}_{*}\left(\gamma_{*} V\right)\right)$ determines the boundary $C=\exp _{\rho(\gamma) q} \circ \operatorname{dev}_{*}\left(\partial H_{\gamma y}\right)$.

Suppose that $\rho(\gamma) m=\exp _{\rho(\gamma) q}\left(\operatorname{dev}_{*}\left(\gamma_{*} X\right)\right)$ does not lie on $C$. Then the geodesic $\alpha(t)=\exp _{\rho(\gamma) q}\left(t \cdot \operatorname{dev}_{*}\left(\gamma_{*} X\right)\right)$ intersects $C$ at some $s<1$. Put $n=\alpha(s)=\exp _{\rho(\gamma) q}$ o 
$\operatorname{dev}_{*}\left(s \cdot \gamma_{*} X\right)$, which lies on $C$. On the other hand, since $s \cdot X \in H_{y}$, $\exp _{y}(s \cdot X)$ is defined. Put $z=\gamma \circ \exp _{y^{\prime}}(s \cdot X) \in \tilde{M}^{\prime}$. Then

$$
\operatorname{dev}(z)=\operatorname{dev} \circ \exp _{\gamma y}\left(s \cdot \gamma_{*} X\right)=\exp _{\rho(\gamma) q} \circ \operatorname{dev}_{*}\left(s \cdot \gamma_{*} X\right)=n
$$

We have the maximal disc $\exp _{n} \operatorname{dev}_{*} D_{R(z)}(z)$ centered at $n$, which intersects the boundary $C$ with the middle of the disc. Let $\exp _{n} \circ \operatorname{dev}_{*}\left(H_{z}\right)$ be the half space containing $\exp _{n} \operatorname{dev}_{*} D_{R(z)}(z)$. Since $\exp _{n} \circ \operatorname{dev}_{*}\left(\partial H_{z}\right)$ contains 0 and the point $w=$ $\exp _{\rho(\gamma) q}\left(\operatorname{dev}_{*}\left(\gamma_{*} V\right)\right) \in C$, we conclude that $w$ sits inside $\exp _{n} \circ \operatorname{dev}_{*}\left(H_{z}\right)$. This contradicts that $w$ is an invisible point. Therefore, the point $\rho(\gamma) m \in \exp _{\rho(\gamma) q}$ 。 $\operatorname{dev}_{*}\left(\partial H_{\gamma y}\right)$ at each $y \in \tilde{M}^{\prime}$. Hence, $J$ is invariant under $\Gamma$.

Concerned with the structure of the boundary $\exp _{q} \circ \operatorname{dev}_{*}\left(\partial H_{y}\right)$, we have the following.

LEMMA 28. Let $V=V^{f} \oplus V^{b}$ be the decomposition for the unique vector $V=$ $V_{y} \in \partial D_{R(y)}(y)$.

(1) If $V^{f} \neq 0$, then $\exp _{q} \circ \operatorname{dev}_{*}\left(\partial H_{y}\right)=\mathbb{R}^{2} \times U$ where $U=\mathbb{F}^{n}$ or $U$ is an affine half space of $\mathbb{F}^{n}$.

(2) If $V^{f}=0$, then $\exp _{q} \circ \operatorname{dev}_{*}\left(\partial H_{y}\right)=\mathbb{R}^{3} \times U$ where $U$ is a $(4 n-1)$-dimensional affine subspace of $\mathbb{F}^{n}$.

Proof. Recall that

$$
\partial H_{y}=\left\{X \in \mathrm{T}_{y} \tilde{M}^{\prime} \mid g_{y}^{\prime}(V, X)=g_{y}^{\prime}\left(V^{f}, X^{f}\right)+g_{y}^{\prime}\left(V^{b}, X^{b}\right)=r^{2}\right\}
$$

for $X=X^{f} \oplus X^{b} \in T_{y} \tilde{M}^{\prime}$.

(1) Suppose that $V^{f} \neq 0$. Let $\operatorname{dev}(y)=q$. The projection $\mathrm{T}_{q} \mathbb{R}^{3} \rightarrow \mathrm{T}_{q} \mathcal{M} \stackrel{\nu_{*}}{\longrightarrow}$ $\mathrm{T}_{\nu(q)} \mathbb{F}^{n}$ induces the map $\nu_{*}: \operatorname{dev}_{*}\left(\partial H_{y}\right) \longrightarrow \nu_{*} \circ \operatorname{dev}_{*}\left(\partial H_{y}\right)$. Put $v_{X}=\nu_{*}\left(\operatorname{dev}_{*}(X)\right)=$ $\nu_{*}\left(\operatorname{dev}_{*}\left(X^{b}\right)\right)$ and $s_{X}=r^{2}-g_{y}^{\prime}\left(V^{b}, X^{b}\right)$. Then the inverse image at $v_{X}$ is a two dimensional affine subspace of $\mathrm{T}_{q} \mathbb{R}^{3}$ :

$$
\nu_{*}^{-1}\left(v_{X}\right)=\left\{\operatorname{dev}_{*}\left(Y^{f}+X^{b}\right) \mid g_{y}^{\prime}\left(V^{f}, Y^{f}\right)=s_{X}\right\}
$$

Since it is a half space as before, $\nu_{*}^{-1}\left(v_{X}\right)$ is perpendicular to $\operatorname{dev}_{*}\left(V^{f}\right)$ and so the fibers $\nu_{*}{ }^{-1}\left(v_{X}\right)$ are parallel to each other. Let $\mathrm{T}_{q} \mathcal{M}=\mathcal{F}_{q} \oplus B_{q}$ be the decomposition for $q \in \mathcal{M}$. Then we note that $\mathcal{F}_{0}$ is the ideal of the nilpotent Lie algebra $T_{0} \mathcal{M}$ generated by the center $\mathbb{R}^{3}$. If $L_{q}$ is the left translation of $\mathcal{M}$, then there is the commutative diagram:

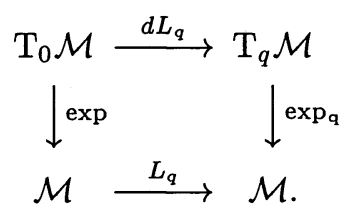

Let $T_{0} \in \mathcal{F}_{0}, S_{0} \in B_{0}$. Since $\mathbb{R}^{3}$ is the center, $\left[T_{0}, S_{0}\right]=0$, it follows that $\exp \left(T_{0}\right)$. $\exp \left(S_{0}\right)=\exp \left(T_{0}+S_{0}\right)$. Choose $T_{0}, S_{0}$ such that $d L_{q}\left(T_{0}\right)=\operatorname{dev}_{*}\left(Y^{f}\right), d L_{q}\left(S_{0}\right)=$ $\operatorname{dev}_{*}\left(X^{b}\right)$. By the commutativity,

$$
\exp _{q}\left(\operatorname{dev}_{*}\left(Y^{f}+X^{b}\right)\right)=\exp T_{0} \cdot \exp _{q}\left(\operatorname{dev}_{*}\left(X^{b}\right)\right)
$$


Let $\nu: \exp _{q} \circ \operatorname{dev}_{*}\left(\partial H_{y}\right) \longrightarrow \exp _{\nu(q)} \circ \nu_{\star} \circ \operatorname{dev}_{\star}\left(\partial H_{y}\right) \subset \mathbb{F}^{n}$ be the projection. Put $z_{X}=\exp _{\nu(q)}\left(v_{X}\right)$. Then the inverse image at $z_{X}$,

$$
\begin{aligned}
\nu^{-1}\left(z_{X}\right) & =\exp _{q}\left(\nu_{*}^{-1}\left(v_{X}\right)\right) \\
& =\left\{\exp T_{0} \cdot \exp _{q}\left(\operatorname{dev}_{*}\left(X^{b}\right)\right) \mid g_{y}^{\prime}\left(T^{-f}, Y^{f}\right)=s_{X}, T_{0}=d L_{q^{-1}} \circ \operatorname{dev}_{*}\left(Y^{f}\right)\right\} .
\end{aligned}
$$

By the above remark, these inverse images $\nu^{-1}\left(z_{X}\right)$ are two dimensional parallel affine subspaces in $\mathbb{R}^{3}$. Hence the subset $\left\{\exp T_{0} \mid T_{0}=d L_{q^{-1}} \circ \operatorname{dev}_{*}\left(Y^{f}\right), g^{\prime}{ }_{y}\left(V^{f}, Y^{f}\right)=\right.$ $\left.s_{X}\right\}$ is a two dimensional vector subgroup $\Xi^{2}$ of $\mathbb{R}^{3}$. Let

$$
\begin{aligned}
W^{\prime} & =\left\{\operatorname{dev}_{*}\left(Z^{b}\right) \in \operatorname{dev}_{*}\left(\partial H_{y}\right) \mid g_{q}\left(\operatorname{dev}_{*}\left(V^{b}\right), \operatorname{dev}_{*}\left(Z^{b}\right)\right)\right. \\
& \left.=r^{2}-g_{q}\left(\operatorname{dev}_{*}\left(V^{f}\right), \operatorname{dev}_{*}\left(Z^{f}\right)\right) \leqq r^{2}\right\}
\end{aligned}
$$

be the subspace of $\mathrm{T}_{q} \mathcal{M}$ for $Z=Z^{f} \oplus Z^{b} \in \mathrm{T}_{y} \tilde{M}^{\prime}$. As $\nu_{*}$ is a local isometry of $\left(B_{q}, g_{q}\right)$ onto $\left(\mathrm{T}_{\nu(q)} \mathbb{F}^{n},\left(g_{0}\right)_{\nu(q)}\right), \nu_{*}$ maps $W^{\prime}$ isometrically onto

$\nu_{*}\left(W^{\prime}\right)=\left\{\nu_{*} \circ \operatorname{dev}_{*}\left(Z^{b}\right) \in \nu_{*} \circ \operatorname{dev}_{*}\left(\partial H_{y}\right) \mid\left(g_{0}\right)_{\nu(q)}\left(\nu_{*} \circ \operatorname{dev}_{*}\left(V^{b}\right), \nu_{*} \circ \operatorname{dev}_{*}\left(Z^{b}\right)\right) \leqq r^{2}\right\}$.

In particular the submanifold $\left(\exp _{q}\left(W^{\prime}\right), g\right)$ is isometric to the affine subspace $\left(\exp _{\nu(q)}\right)^{\circ}$ $\left.\nu_{*}\left(W^{\prime}\right), g_{0}\right)$ of $\mathbb{F}^{n}$. Put $U=\exp _{\nu(q)} \circ \nu_{*}\left(W^{\prime}\right)$. Then $U$ is either $\mathbb{F}^{n}$ or an affine half space according to whether $V^{b}=0$ or $V^{b} \neq 0$. Since $\left(\exp _{q}\left(W^{\prime}\right), g\right)$ is a flat submanifold of $\mathcal{M}$, we have that $\exp _{q}\left(W^{\prime}\right)=(\alpha, U)$ for some $\alpha \in \mathbb{R}^{3}$. If we note that $\exp _{q} \circ \operatorname{dev}_{*}\left(\partial H_{y}\right)=\mathbb{R}^{2} \cdot \exp _{q}\left(W^{\prime}\right)$ which contains 0 , then $\alpha \in \mathbb{R}^{2}$ and hence $\exp _{q} \circ \operatorname{dev}_{*}\left(\partial H_{y}\right)=\mathbb{R}^{2} \times U$.

For $(2)$, as $V^{f}=0, g_{q}\left(\operatorname{dev}_{*}\left(V^{b}\right), \operatorname{dev}_{*}\left(Z^{b}\right)\right)=\left(g_{0}\right)_{\nu(q)}\left(\nu_{*} \circ \operatorname{dev}_{*}\left(V^{b}\right), \nu_{*} \circ \operatorname{dev}_{*}\left(Z^{b}\right)\right)$ $=r^{2}$. Then $U=\exp _{\nu(q)} \circ \nu_{*}\left(W^{\prime}\right)=\nu \circ \exp _{q} \circ \operatorname{dev}_{*}\left(\partial H_{y}\right)$ is a $(4 n-1)$-dimensional affine subspace of $\mathbb{F}^{n}$. It is easy to see that $\exp _{q} \circ \operatorname{dev}_{*}\left(\partial H_{y}\right)$ is a principal $\mathbb{R}^{3}$-bundle over $U$. Hence, $\exp _{q} \circ \operatorname{dev}_{*}\left(\partial H_{y}\right)=\mathbb{R}^{3} \times U$.

As $J=\bigcap_{y \in \tilde{M}^{\prime}} \exp _{q} \circ \operatorname{dev}_{*}\left(\partial H_{y}\right)$ by the definition, $J$ is an affine subspace of $(\mathcal{M}, \nabla)$, i.e., $J=\mathbb{R}^{k} \times W$ where $\mathbb{R}^{k}$ is a vector subspace of $\mathbb{R}^{3}$ and $W$ is an affine subspace of $\mathbb{R}^{4 n}=\mathbb{F}^{n}$. In particular we have the following.

Corollary 29.

(i) If $\bar{J}=J$, then $J$ is a single point $\{0\}$ in $\mathcal{M}$.

(ii) If $\bar{J}=J \cup\{\infty\}$, then $\bar{J}$ is an $\ell(\geqq 1)$-dimensional sphere $S^{\ell}$.

Proof of Theorem 19. (I) $\bar{J}=J$. Then $J=\{0\}$, which implies that the holonomy group $\Gamma \subset \mathrm{Sp}(n) \cdot \operatorname{Sp}(1) \times \mathbb{R}^{+}$.

$(\mathbf{I})_{1} \operatorname{dev}^{-1}(\infty)=\emptyset$. Then $\tilde{M}=\tilde{M}^{\prime}$ and dev $: \tilde{M} \rightarrow \mathcal{M}-\{0\}=\mathbb{R}^{+} \times S^{4 n+2}$ by Corollary 24. As $M$ is compact, dev $: \tilde{M} \longrightarrow \mathbb{R}^{+} \times S^{4 n+2}$ is homeomorphic so that $M$ is finitely covered by a Hopf manifold $S^{1} \times S^{4 n+2}$.

$(\mathbf{I})_{2} \operatorname{dev}^{-1}(\infty) \neq \emptyset$. Then the developing map satisfies that dev $: \tilde{M} \longrightarrow S^{4 n+3}-$ $\{0\}$. Replace $S^{4 n+3}-\{0\}$ for the role of $\mathcal{M}=S^{4 n+3}-\{\infty\}$. As a consequence, we show this case does not occur. In fact, put $\mathcal{M}^{\prime}=S^{4 n+3}-\{0\}$. If $\tilde{M}$ is complete, then $\operatorname{dev}: \tilde{M} \longrightarrow \mathcal{M}^{\prime}$ is homeomorphic. Then $\Gamma$ acts freely on $\mathcal{M}^{\prime}$, while $\Gamma$ has a fixed point $\{\infty\}$ inside $\mathcal{M}^{\prime}$. So $\tilde{M}$ is incomplete. The same argument as above shows that there is a $\Gamma$-invariant affine subspace $J^{\prime}$ which is outside the developing image $\operatorname{dev}(\tilde{M})$. If $\partial \operatorname{dev}(\tilde{M})$ is the boundary of the developing image in $S^{4 n+3}$, then $\partial \operatorname{dev}(\tilde{M})$ is a $\Gamma$ invariant closed subset containing at least two points. Recall the limit set $L(\Gamma)$ of $\Gamma$ in $S^{4 n+3}$, which is defined to be the boundary of the closure of the orbit $\Gamma \cdot w$ for a point 
$w \in \mathbb{H}_{\mathbb{F}}^{n+1}$. (Compare [8].) By minimality, $L(\Gamma) \subset \partial \operatorname{dev}(\tilde{M})$. Thus we have that $\operatorname{dev}: \tilde{M} \longrightarrow S^{4 n+3}-L(\Gamma)$. As $\Gamma \subset \operatorname{Sp}(n) \cdot \operatorname{Sp}(1) \times \mathbb{R}^{+}$in our case $(\mathbf{I}), L(\Gamma)=\{0, \infty\}$ and so the developing image $\operatorname{dev}(\tilde{M})$ misses the point $\{\infty\}$. This contradicts the hypothesis $\left(\mathbf{I}_{2}\right)$.

(II) $\bar{J}=J \cup\{\infty\} . \quad$ By Lemma 26 and Corollary $29(i i), \operatorname{dev}: \tilde{M} \longrightarrow \mathcal{M}-J$ where $J$ is an $\ell(\geqq 1)$-dimensional affine flat subspace of $\mathcal{M}$.

We define a $\bar{\Gamma}$-invariant Riemannian metric on $\mathcal{M}-J$. Let $Z$ be a vector field which assigns to each $p \in \mathcal{M}-J$ the vector $Z(p)$ from $p$ to $J$, which is perpendicular to $J$ with respect to the left invariant metric $g$ on $\mathcal{M}$. Such a vector $Z(p)$ is uniquely determined because $J$ is an affine flat subspace $(\mathcal{M}, \nabla), Z(p)$ is the shortest vector which meets $J$. Moreover, since $J$ is invariant under $\Gamma$, the vector field $Z$ is $\Gamma$-invariant. As in the Remark 22, let $X=X^{f} \oplus X^{b}$ (resp. $Y=Y^{f} \oplus Y^{b}$ ) be a decomposition for vectors $X, Y \in \mathrm{T}_{x}(\mathcal{M}-J)$. We define a Riemannian metric:

$$
\tilde{h}_{p}(X, Y)=\frac{g_{x}\left(X^{f}, Y^{f}\right)}{\left\|Z^{f}(p)\right\|^{2}+\left\|Z^{b}(p)\right\|^{4}}+\frac{g_{x}\left(X^{b}, Y^{b}\right)}{\left\|Z^{f}(p)\right\|+\left\|Z^{b}(p)\right\|^{2}} .
$$

By the dilation property $(*),(* *)$ of $(3.2), \tilde{h}$ is a Riemannian metric on $\mathcal{M}-J$ which is invariant under $\Gamma$. The pullback by dev defines a $\pi$-invariant Riemannian metric $\operatorname{dev}^{*} \tilde{h}$ on $\tilde{M}$ such that dev $: \tilde{M} \longrightarrow \mathcal{M}-J$ is a local isometry. Then $M$ becomes a compact Riemannian manifold induced by $\operatorname{dev}^{*} \tilde{h}$. As a consequence $\tilde{M}$ is complete so that dev $: \tilde{M} \longrightarrow \mathcal{M}-J$ is a covering map. Since $J$ is an $\ell$-dimensional affine flat subspace of $\mathcal{M}$ with $1 \leqq \ell \leqq 4 n+2$, there are the following cases: (i) if $\operatorname{dim} J \leqq 4 n$, then $\operatorname{dev}: \tilde{M} \longrightarrow \mathcal{M}-J$ is homeomorphic; (ii) if $\operatorname{dim} J=4 n+1$, then $\operatorname{dev}: \tilde{M} \longrightarrow \overline{\bar{M}}-J$ is a covering map; (iii) if $\operatorname{dim} J=4 n+2$, then $\operatorname{dev}$ is a homeomorphism of $\tilde{M}$ onto a connected component of $\mathcal{M}-J$.

For the cases (i), (iii), $\Gamma$ is discrete in $\operatorname{Sim}(\mathcal{M})$, so either $L(\Gamma)=\{\infty\}$ or $L(\Gamma)=$ $\{0, \infty\}$. Since $\partial \operatorname{dev}(\tilde{M})$ contains more than one point, we have that $L(\Gamma) \subset \partial \operatorname{dev}(\tilde{M})$. Thus dev $: \tilde{M} \longrightarrow S^{4 n+3}-L(\Gamma)$ is homeomorphic for which $S^{4 n+3}-L(\Gamma)=\mathcal{M}$ or $S^{4 n+3}-L(\Gamma)=\mathcal{M}-\{0\}$ respectively. Hence $\operatorname{dev}(\tilde{M}) \cap J-\{0\} \neq \emptyset$. This contradicts Lemma 26 under the hypothesis (II).

For the case (ii), note that $J=\mathbb{R}^{k} \times \mathbb{R}^{4 n+1-k}$ such that $\bar{J}=S^{4 n+1}$.

(ii) ${ }_{1}$ Suppose that $k=3$, i.e., $J=\mathbb{R}^{3} \times \mathbb{R}^{4 n-2}$. Then $\nu^{-1}\left(\mathbb{R}^{4 n-2}\right)=J$ from (1.4). Moreover, $\mathcal{M}-J=S^{4 n+3}-\bar{J}=S^{4 n+3}-S^{4 n+1} \approx B^{4 n+2} \times S^{1}$ where $B^{4 n+2}$ is a $(4 n+2)$-dimensional ball. Let $X$ be the universal covering space of $\mathcal{M}-J$. The developing map dev lifts to a homeomorphism dev : $\tilde{M} \longrightarrow X$ which maps $\pi$ onto a subgroup $\tilde{\Gamma}$ acting properly discontinuously and freely on $X$. In particular, $M \approx X / \tilde{\Gamma}$ is compact. However if we note that the action $(\tilde{\Gamma}, X)$ is a lift of the action $\left(\Gamma, S^{4 n+3}-S^{4 n+1}\right)$, then $X / \tilde{\Gamma}$ cannot be compact by (3) of Lemma 3 .

(ii) 2 Suppose that $J=\mathbb{R}^{2} \times \mathbb{R}^{4 n-1}$ or $J=\mathbb{R}^{1} \times \mathbb{F}^{n}$. Consider the case $J=$ $\mathbb{R}^{2} \times \mathbb{R}^{4 n-1}$. Recall that an element $g$ of $\operatorname{Sim}(\mathcal{M})$ has the form:

$$
g=(\alpha, x) \cdot(A \cdot g, t) \text {. }
$$

For

$$
\left(\begin{array}{l}
\beta \\
z
\end{array}\right) \in \mathcal{M}
$$

the action of $g$ satisfies that

$$
g\left(\begin{array}{c}
\beta \\
z
\end{array}\right)=\left(\begin{array}{c}
\alpha+t^{2} g \cdot \beta \cdot g^{-1}+\operatorname{Im}<x, t A z \cdot g^{-1}> \\
x+t A z \cdot g^{-1}
\end{array}\right) .
$$


The subspace $\mathbb{R}^{4 n-1}$ contains at least one quaternionic subspace $\mathbb{F}^{1}$. As $\Gamma$ leaves $J$ invariant for which $\beta \in \mathbb{R}^{2}$ and $z \in \mathbb{R}^{4 n-1}$ can be chosen arbitrarily, it follows that $x=0$. Thus $\Gamma$ lies in the subgroup $\left(\mathbb{R}^{2}, 0\right) \rtimes \mathrm{Sp}(n) \cdot \mathrm{SO}(2) \times \mathbb{R}^{+}$. (Note that this group does not preserve $\mathbb{R}^{3} \times \mathbb{R}^{n}$, although $\Gamma$ acts invariantly on $\mathbb{R}^{3}=\left(\mathbb{R}^{3}, 0\right)$ as similarity transformations.)

Let $\gamma=(\alpha, 0) \cdot(A \cdot g, t)$ be an element of $\left(\mathbb{R}^{2}, 0\right) \rtimes \mathrm{Sp}(n) \cdot \mathrm{SO}(2) \times \mathbb{R}^{+}$. Then, for $(\beta, x) \in \mathcal{M}=\mathbb{R}^{3} \times \mathbb{F}^{n}$

$$
\begin{aligned}
\gamma\left(\begin{array}{c}
\beta \\
x
\end{array}\right) & =\left(\begin{array}{c}
\alpha+t^{2} g \cdot \beta \cdot g^{-1} \\
t \cdot A x \cdot g^{-1}
\end{array}\right)=\left(\begin{array}{c}
\alpha+t^{2} \cdot B_{1} \beta \\
t \cdot B_{2} x
\end{array}\right) \\
& =\left(\begin{array}{c}
\alpha \\
0
\end{array}\right)+\left(\begin{array}{lr}
t^{2} \cdot B_{1} & 0 \\
0 & t \cdot B_{2}
\end{array}\right)\left(\begin{array}{c}
\beta \\
x
\end{array}\right) .
\end{aligned}
$$

Here, a matrix $B_{1} \in \mathrm{SO}(2)$ is the conjugate of $g$ and a matrix $B_{2} \in \mathrm{SO}(4 n)$ is $A \cdot g$ under the identification $\mathbb{F}^{n}=\mathbb{R}^{4 n}$.

Let $f: \mathcal{M}=\mathbb{R}^{3} \times \mathbb{R}^{4 n} \longrightarrow \mathbb{R}^{3} \times \mathbb{R}^{4 n}$ be the diffeomorphism defined by $f(\alpha, x)=$ $(\alpha, x|x|)$ where $|\cdot|$ is the euclidean norm. Then it is easy to check that

$$
f\left(\gamma\left(\begin{array}{c}
\beta \\
x
\end{array}\right)\right)=\left(\begin{array}{lr}
t^{2} \cdot B_{1} & 0 \\
0 & t^{2} \cdot B_{2}
\end{array}\right) \cdot f\left(\left(\begin{array}{l}
\beta \\
x
\end{array}\right)\right)+\left(\begin{array}{c}
\alpha \\
0
\end{array}\right) .
$$

There is a homomorphism $\tau: \Gamma \longrightarrow\left(\mathbb{R}^{2}, 0\right) \rtimes(\mathrm{SO}(2) \times \operatorname{SO}(4 n)) \times \mathbb{R}^{+} \subset \operatorname{Sim}\left(\mathbb{R}^{4 n+3}\right)$ such that $f \circ \gamma=\tau(\gamma) \circ f$. We have an equivariant diffeomorphism:

$$
(\Gamma, \mathcal{M}) \stackrel{f}{\longrightarrow}\left(\tau(\Gamma), \mathbb{R}^{4 n+3}\right) .
$$

The pair $(\tau \circ \rho, f \circ \mathrm{dev}):(\pi, \tilde{M}) \longrightarrow\left(\operatorname{Sim}\left(\mathbb{R}^{4 n+3}\right), \mathbb{R}^{4 n+3}\right)$ gives a similarity structure on $M$ in which $f$ o dev misses $f(J)$. The theorem of Fried [12] says that the developing map $f \circ \operatorname{dev}: \tilde{M} \longrightarrow \mathbb{R}^{4 n+3}$ of a compact incomplete similarity manifold $M$ misses exactly one point $\{0\}$ (up to conjugacy). Hence $f(J)=\{0\}$, which is impossible by $\operatorname{dim} J=4 n+1$. The same argument can be applied to the case that $J=\mathbb{R}^{1} \times \mathbb{F}^{n}$. As a consequence, (II) does not occur. This completes the proof of Theorem 19.

\section{Geometric superrigidity.}

4.1. Geometric rigidity on spherical pseudo-quaternionic manifolds. Margulis has shown that: Let $G$ be a connected semisimple Lie group with trivial center and has no compact factor. Given an irreducible lattice $\Gamma$ of $G$ and a homomorphism $\rho: \Gamma \longrightarrow G^{\prime}$ where $G^{\prime}$ is a semisimple Lie group with trivial center and without compact factor, $\rho$ extends to a homomorphism from $G$ to $G^{\prime}$ provided that the real rank of $G$ is at least two and $\rho(\Gamma)$ is Zariski dense in $G^{\prime}$. Note that a connected semisimple Lie group with trivial center supports a real algebraic structure. (Compare [47].) This sort of result is called Margulis' superrigidity and the question is left to the rank one semisimple Lie groups, namely the real (resp. complex, quaternionic, Cayley) hyperbolic groups. It is known that the Margulis' superrigidity is false for the real hyperbolic case, for instance, because of the existence of bending (= a nontrivial deformation of Fuchsian groups in higher dimensions). On the other hand, Kevin Corlette [11] has proved the Margulis' superrigidity affirmatively for the cases $G$ of the isometry group $\operatorname{PSp}(n, 1)(n \geqq 2)$ and the isometry group $F_{4}^{-20}$ of the hyperbolic Cayley plane. See also [33]. 
As in (1.3) we have a compact spherical pseudo-quaternionic manifold

$$
\mathrm{Sp}(m) \times \Delta \mathrm{Sp}(1) \times \mathrm{Sp}(n-m) \backslash \mathrm{Sp}(m, 1) \times \mathrm{Sp}(n-m+1) / \Gamma=S^{4 n+3}-S^{4 m-1} / \Gamma,
$$

in which the fundamental group $\Gamma$ is virtually isomorphic to a discrete uniform subgroup of $\operatorname{PSp}(m, 1)$. In this section we prove the following rigidity.

THEOREM 30. Let $M$ be a compact spherical pseudo-quaternionic $(4 n+3)$ manifold whose fundamental group is virtualy isomorphic to a discrete uniform subgroup of $\operatorname{PSp}(m, 1)$ for some $2 \leqq m \leqq n$. Then $M$ is pseudo-quaternionically isomorphic to the double coset space

$$
\operatorname{Sp}(m) \times \Delta \operatorname{Sp}(1) \times \operatorname{Sp}(n-m) \backslash \operatorname{Sp}(m, 1) \times \operatorname{Sp}(n-m+1) / \Gamma,
$$

where $m=2, \cdots, n$.

Proof. Let $M$ be a compact spherical pseudo-quaternionic $(4 n+3)$-manifold. Then there exists a developing pair $(\rho, \mathrm{dev}):\left(\pi_{1}(M), \tilde{M}\right) \longrightarrow\left(\operatorname{PSp}(n+1,1), S^{4 n+3}\right)$. Put $\pi=\pi_{1}(M), \rho(\pi)=\Gamma$. We have the holonomy representation

$$
\rho: \pi \longrightarrow \Gamma \subset \operatorname{PSp}(n+1,1) \text {. }
$$

By the hypothesis, passing to a subgroup of finite inclex, $\pi$ is isomorphic to a discrete uniform subgroup of $\operatorname{PSp}(m, 1)$ for some $2 \leqq m \leqq n$. We may assume that $\pi \subset$ $\operatorname{PSp}(m, 1)$. If $\Gamma$ is amenable, then the classification theorem 19 shows that $\pi$ is virtually nilpotent. This case does not occur by the hypothesis.

Let $\mathcal{A}(\Gamma)$ be the Zariski closure (real algebraic closure) of $\Gamma$ in $\operatorname{PSp}(n+1,1)$. Then by Theorem 4.4 .2 of [8] shows that either $\mathcal{A}(\Gamma)=\operatorname{PSp}(n+1,1)$ or $\mathcal{A}(\Gamma)$ leaves a proper totally geodesic subspace in $\mathbb{H}_{\mathbb{F}}^{n+1}$.

(i) Suppose that $\mathcal{A}(\Gamma)=\operatorname{PSp}(n+1,1)$. Then $\Gamma$ is Zariski dense in $\operatorname{PSp}(n+1,1)$. The superrigidity by Corlette implies that $\rho$ extends to a continuous homomorphism $\varphi: \operatorname{PSp}(m, 1) \longrightarrow \operatorname{PSp}(n+1,1)$. Since $\varphi$ is analytic (cf. [15]), the image $\varphi(\operatorname{PSp}(m, 1))$ is a connected Lie subgroup of $\operatorname{PSp}(n+1,1)$. As $\Gamma \subset \varphi(\operatorname{PSp}(m, 1))$ does not leave any proper totally geodesic subspace in $\mathbb{H}_{\mathbb{F}}^{n+1}, \varphi(\operatorname{PSp}(m, 1))=\operatorname{PSp}(n+1,1)$ and so $m=n+1$. Since $m \leqq n$ by our hypothesis, this is impossible.

(ii) Suppose that $\mathcal{A}(\Gamma)$ leaves a proper totally geodesic subspace in $\mathbb{H}_{\mathbb{F}}^{n+1}$. A proper totally geodesic subspace in $\mathbb{H}_{\mathbb{F}}^{n+1}$ is isometric to $\mathbb{H}_{\mathbb{K}}^{k}(\mathbb{K}=\mathbb{R}, \mathbb{C}$ or $\mathbb{F}, 1 \leqq$ $k \leqq n), \mathbb{H}_{\mathbb{R}}^{n+1}, \mathbb{H}_{\mathbb{C}}^{n+1}$, or a 3-dimensional $\mathbb{R}$-subspace $\mathbb{H}^{1}(\mathrm{I})$. (Compare [8].) Note that $\mathbb{H}^{1}(\mathrm{I})$ is orthogonal to $\mathbb{H}_{\mathbb{R}}^{1}$ in $\mathbb{H}_{\mathbb{F}}^{1}$ and so isometric to $\mathbb{H}_{\mathbb{R}}^{3}$. If $\mathcal{A}(\Gamma)$ leaves invariant a proper subspace $\mathbb{H}_{\mathbb{K}}^{k}(\mathbb{K}=\mathbb{R}, \mathbb{C}, \mathbb{F}, k \leqq n)$, then $\mathcal{A}(\Gamma)$ leaves also $\mathbb{H}_{\mathbb{F}}^{k}$ invariant. Thus $\mathcal{A}(\Gamma)$ preserves $S^{4 k-1}=\partial \mathbb{H}_{\mathbb{F}}^{k}$. The subgroup of $\mathrm{PSp}(n+1,1)$ preserving $S^{4 k-1}$ is isomorphic to $\operatorname{Sp}(k, 1) \cdot \operatorname{Sp}(n-k+1)$. In particular, $\mathcal{A}(\Gamma) \subset \operatorname{Sp}(k, 1) \cdot \operatorname{Sp}(n-k+1)$ for $k \leqq n$. Then recall that there is a $\Gamma$-invariant homogeneous Riemannian metric on $S^{4 n+3}-S^{4 k-1}$ from (1.3).

If $\mathcal{A}(\Gamma)$ leaves invariant $\mathbb{H}_{\mathbb{K}}^{n+1}(\mathbb{K}=\mathbb{R}, \mathbb{C})$, then $\mathcal{A}(\Gamma)$ preserves $S^{n}=\partial \mathbb{H}_{\mathbb{R}}^{n+1}$ (resp. $S^{2 n+1}=\partial \mathbb{H}_{\mathbb{C}}^{n+1}$ ). By Proposition 5, Corollary 6, $\mathcal{A}(\Gamma) \subset \mathrm{PO}(n+1,1) \times \mathrm{SO}(3)$ or $\mathcal{A}(\Gamma) \subset \mathrm{P}\left(\mathrm{U}(n+1,1) \cdot S^{1}\{ \pm 1, \pm j\}\right)$ respectively. Moreover $S^{4 n+3}-S^{n}$ (resp. $\left.S^{4 n+3}-S^{2 n+1}\right)$ admits a $\Gamma$-invariant complete Riemannian metric.

If $\mathcal{A}(\Gamma)$ preserves $\mathbb{H}^{1}(\mathrm{I})$, then it leaves also $\mathbb{H}_{\mathbb{F}}^{1}$ invariant. As $\mathbb{H}^{1}(\mathrm{I})$ is isometric to $\mathbb{H}_{\mathbb{R}}^{3}$, the subgroup of $\operatorname{PSp}(n+1,1)$ preserving $\mathbb{H}^{1}(\mathrm{I})$ is isomorphic to $\operatorname{PSL}(2, \mathbb{C}) \times \operatorname{Sp}(n)$ where $\operatorname{PSL}(2, \mathbb{C})=\operatorname{Iso}\left(\mathbb{H}_{\mathbb{R}}^{3}\right)$. Thus $\mathcal{A}(\Gamma) \subset \operatorname{PSL}(2, \mathbb{C}) \times \operatorname{Sp}(n)$ which leaves invariant $S^{3}=\partial \mathbb{H}_{F}^{1}$. 
Let $\tau: \operatorname{Sp}(k, 1) \cdot \operatorname{Sp}(n-k+1) \longrightarrow \mathrm{PSp}(k, 1)$ be the canonical projection. Similarly for $\tau: \operatorname{PSL}(2, \mathbb{C}) \times \operatorname{Sp}(n) \longrightarrow \mathrm{PSL}(2, \mathbb{C}), \tau: \mathrm{PO}(n+1,1) \times \mathrm{SO}(3) \longrightarrow \mathrm{PO}(n+1,1)$ or $\tau: \mathrm{P}\left(\mathrm{U}(n+1,1) \cdot S^{1}\{ \pm 1, \pm j\}\right) \rightarrow \mathrm{PU}(n+1,1)$ respectively. Suppose that $H$ is one of $\operatorname{PSp}(k, 1), \operatorname{PSL}(2, \mathbb{C}), \operatorname{PO}(n+1,1)$, or $\operatorname{PU}(n+1,1)$.

Since $\tau$ is a proper map and $\mathcal{A}(\Gamma)$ (with finitely many components) leaves the proper totally geodesic subspace, Theorem 4.4 .1 of [8] implies that $\tau(\mathcal{A}(\Gamma))=H$. In particular, $\tau(\Gamma)$ is Zariski dense in $H$. We obtain a homomorphism $\tau \circ \rho: \pi \longrightarrow H$ with Zariski dense image $\tau(\Gamma)$. As $H$ is a noncompact simple Lie group and without center, applying the Corlette's superrigidity, $\tau \circ \rho$ extends to a continuous homomorphism $\Psi$ : $\operatorname{PSp}(m, 1) \longrightarrow H$. As in (i), we obtain that $\Psi(\operatorname{PSp}(m, 1))=H$. Moreover $\operatorname{PSp}(m, 1)$ has no normal subgroup, so $\Psi: \operatorname{PSp}(m, 1) \longrightarrow H$ is an isomorphism. Therefore $2 \leqq$ $m=k \leqq n$. As a consequence, $\Gamma$ is a discrete uniform subgroup of $\operatorname{Sp}(m, 1) \cdot \operatorname{Sp}(n-$ $m+1)$. In particular we have $\Lambda(\Gamma)=L(\operatorname{Sp}(m, 1) \cdot \operatorname{Sp}(n-m+1))=S^{4 m-1}$. Consider the developing map

$$
\operatorname{dev}: \tilde{M}-\operatorname{dev}^{-1}\left(S^{4 m-1}\right) \longrightarrow S^{4 n+3}-S^{4 m-1}
$$

for which the homogeneous Riemannian metric $h$ on $S^{4 n+3}-S^{4 m-1}$ (cf. 1.3) induces a Riemannian submersion:

$$
S^{4(n-m)+3} \rightarrow\left(\operatorname{Sp}(m, 1) \cdot \operatorname{Sp}(n-m+1), S^{4 n+3}-S^{4 m-1}, h\right) \stackrel{\nu}{\longrightarrow}\left(\operatorname{PSp}(m, 1), \mathbb{H}_{\mathbb{F}}^{m}, \hat{h}\right) .
$$

Here $\hat{h}$ is the hyperbolic metric on $\mathbb{H}_{\mathbb{F}}^{m}$. Let $\operatorname{dev}^{*} h$ be the induced Riemannian metric on $\tilde{M}-\operatorname{dev}^{-1}\left(S^{4 m-1}\right)$ which is invariant under $\pi$.

(iii) We prove that $\operatorname{dev}^{*} h$ on $\tilde{M}-\operatorname{dev}^{-1}\left(S^{4 m-1}\right)$ is complete. Recall that $\nu$ maps the boundary $S^{4 m-1}=\partial\left(S^{4 n+3}-S^{4 m-1}\right)$ identically onto $S^{4 m-1}=\partial \mathbb{H}_{\mathbb{F}}^{m}$ under the projection $\operatorname{Sp}(n-m) \rightarrow S^{4 n+3} \stackrel{\nu}{\longrightarrow} D^{4 m}$.

Let $\left\{x_{i}\right\}$ be a Cauchy sequence in $\tilde{M}-\operatorname{dev}^{-1}\left(S^{4 m-1}\right)$ with respect to the induced metric $\operatorname{dev}^{*} h$. We may suppose that $\operatorname{dev}^{-1}\left(S^{4 m-1}\right) \neq \emptyset$. Let $\rho^{*}$ (resp. $\rho$ ) be the distance function on $\tilde{M}-\operatorname{dev}^{-1}\left(S^{4 m-1}\right)$ (resp. $\left.S^{4 n+3}-S^{4 m-1}\right)$, and $\hat{\rho}$ be the (hyperbolic) distance function on $\mathbb{H}_{\mathbb{F}}^{m} . A s \operatorname{dev}^{-1}\left(S^{4 m-1}\right)$ is invariant under $\pi$, $M$ decomposes into the union $\left(\tilde{M}-\operatorname{dev}^{-1}\left(S^{4 m-1}\right)\right) / \pi$ and $\mathrm{dev}^{-1}\left(S^{4 m-1}\right) / \pi$ where $\operatorname{dev}^{-1}\left(S^{4 m-1}\right) / \pi$ consists of a finite number of compact submanifolds. If $P: \tilde{M} \longrightarrow M$ is a covering map, then the sequence $\left\{P\left(x_{i}\right)\right\}$ has an accumulation point $y$ (after passing to a subsequence). Choose $\tilde{y} \in \mathrm{dev}^{-1}\left(S^{4 m-1}\right)$ with $P(\tilde{y})=y$. There exists a neighborhood $W$ of $\tilde{y}$ in $\tilde{M}$ such that the closure $\bar{W}$ is compact. Moreover, $P: \bar{W} \longrightarrow P(\bar{W})$ and dev $: \bar{W} \longrightarrow \operatorname{dev}(\bar{W})$ are diffeomorphic. As $y \in P(W)$, there exist elements $\left\{\gamma_{i}\right\} \in \pi$ such that $\left\{\gamma_{i} x_{i}\right\} \in W$ for $i \geqq L$ where $L$ is a sufficiently large number. We have $\lim \gamma_{i} x_{i}=\tilde{y}$. Since $\left\{x_{i}\right\}$ is Cauchy in $\left(\tilde{M}-\operatorname{dev}^{-1}\left(S^{4 m-1}\right), \rho^{*}\right)$, for each integer $n$, there exists an integer $\lambda(n)$ satisfying that if $i, j \geqq \lambda(n), \rho^{*}\left(x_{i}, x_{j}\right)<\frac{1}{n}$. Let $B_{\frac{1}{n}}\left(x_{\lambda(n)}\right)$ be the ball of radius $\frac{1}{n}$ centered at $x_{\lambda(n)}$ in $\tilde{M}-\operatorname{dev}^{-1}\left(S^{4 m-1}\right)$. In particular,

$$
\left\{x_{i}\right\} \in B_{\frac{1}{n}}\left(x_{\lambda(n)}\right) \text { for } i \geqq \lambda(n) .
$$

As $\lambda(n)$ increases as $n$ does, we can assume that $\lambda(n) \geqq n$ for $n \geqq N$ where $N$ is a sufficiently large number with $N>L$. In particular we note that $\left\{\bar{\gamma}_{\lambda(n)} \cdot x_{\lambda(n)}\right\} \in W$ for $n \geqq N$.

Then we show that there is an integer $m$ such that $B_{\frac{1}{m}}\left(\gamma_{\lambda(m)} \cdot x_{\lambda(m)}\right) \subset W$. Suppose not. Put $\partial^{\prime} W=\partial \bar{W} \cap\left(\tilde{M}-\operatorname{dev}^{-1}\left(S^{4 m-1}\right)\right)$. Then for each $n \geqq N$, there 
is a point $z_{\lambda(n)}$ of $B_{\frac{1}{n}}\left(\gamma_{\lambda(n)} \cdot x_{\lambda(n)}\right)$ lying on $\partial^{\prime} W$. Thus we have that $\rho^{*}\left(\gamma_{\lambda(n)} \cdot x_{\lambda(n)}, z_{\lambda(n)}\right) \leqq \frac{1}{n}$.

In general, for every $z \in \partial^{\prime} W \subset \tilde{M}-\operatorname{dev}^{-1}\left(S^{4 m-1}\right)$, the metrics satisfy

$$
\begin{aligned}
\hat{\rho}\left(\nu \circ \operatorname{dev}\left(\gamma_{\lambda(n)} \cdot x_{\lambda(n)}\right), \nu \circ \operatorname{dev}(z)\right) & \leqq \rho\left(\operatorname{dev}\left(\gamma_{\lambda(n)} \cdot x_{\lambda(n)}\right), \operatorname{dev}(z)\right) \\
& \leqq \rho^{*}\left(\gamma_{\lambda(n)} \cdot x_{\lambda(n)}, z\right) .
\end{aligned}
$$

In particular,

$$
\hat{\rho}\left(\nu \circ \operatorname{dev}\left(\gamma_{\lambda(n)} \cdot x_{\lambda(n)}\right), \nu \circ \operatorname{dev}\left(z_{\lambda(n)}\right)\right) \leqq \frac{1}{n} .
$$

As $\lim \gamma_{i} \cdot x_{i}=\tilde{y}$, and $\nu$ extends to a map identically on the boundary, $\nu \circ \operatorname{dev}\left(\gamma_{\lambda(n)}\right.$. $\left.x_{\lambda(n)}\right) \longrightarrow \nu \circ \operatorname{dev}(\tilde{y}) \in \nu\left(S^{4 m-1}\right)=S^{4 m-1}$. On the other hand, $\bar{W}$ is a compact neighborhood of $\tilde{y}$ in $\tilde{M}$ and $\operatorname{dev}: \bar{W} \longrightarrow \operatorname{dev}(\bar{W})$ is diffeomorphic. In particular, $\nu \circ \operatorname{dev}(\tilde{y}) \in S^{4 m-1}-\partial \operatorname{dev}(\bar{W})$.

Since $z_{\lambda(n)} \in \partial^{\prime} W,\left\{z_{\lambda(n)}\right\}$ has an accumulation point $z$ in $\partial \bar{W}$. Passing to a subsequence if necessary, it follows that $\operatorname{dev}\left(z_{\lambda(n)}\right) \longrightarrow \operatorname{dev}(z) \in \partial \operatorname{dev}(\bar{W})$. Therefore, as $\nu \circ \operatorname{dev}\left(\tilde{M}-\operatorname{dev}^{-1}\left(S^{4 m-1}\right)\right) \subset \mathbb{H}_{F}^{m}$, it follows either $\nu \circ \operatorname{dev}(z) \in \mathbb{H}_{\mathbb{P}}^{m}$ or $\nu \circ \operatorname{dev}(z) \in \partial \operatorname{dev}(\bar{W}) \cap S^{4 m-1}$, i.e., $\nu \circ \operatorname{dev}(z) \neq \nu \circ \operatorname{dev}(\tilde{y})$. In each case,

$$
\lim _{n \rightarrow \infty} \hat{\rho}\left(\nu \circ \operatorname{dev}\left(\gamma_{\lambda(n)} \cdot x_{\lambda(n)}\right), \nu \circ \operatorname{dev}\left(z_{\lambda(n)}\right)\right)=\infty,
$$

which is impossible by $(*)$. Hence we obtain that $B_{\frac{1}{m}}\left(\gamma_{\lambda(m)} \cdot x_{\lambda(m)}\right) \subset W$ for some $m$.

Since $\left\{x_{i}\right\}_{i \geqq \lambda(m)} \in B_{\frac{1}{m}}\left(x_{\lambda(m)}\right)$, the isometry $\gamma_{\lambda(m)}$ (with respect to $\rho^{*}$ ) shows that $\left\{\gamma_{\lambda(m)} \cdot x_{i}\right\}_{i \geqq \lambda(m)} \in B_{\frac{1}{m}}\left(\gamma_{\lambda(m)} \cdot x_{\lambda(m)}\right)$. As $\bar{W}$ is compact, there is a point $w \in \bar{W}$ such that $\lim _{i \rightarrow \infty} \gamma_{\lambda(m)} \cdot x_{i}=w$. Therefore $\lim _{i \rightarrow \infty} x_{i}=\gamma_{\lambda(m)}^{-1} \cdot w$ for which $\operatorname{dev}\left(\gamma_{\lambda(m)}^{-1} \cdot w\right)=\lim _{i \rightarrow \infty} \operatorname{dev}\left(x_{i}\right)$. Since the sequence of images $\left\{\operatorname{dev}\left(x_{i}\right)\right\}$ is also Cauchy in the complete metric space $S^{4 n+3}-S^{4 m-1},\left\{\operatorname{dev}\left(x_{i}\right)\right\}$ has a limit point in $S^{4 n+3}-S^{4 m-1}$, which therefore implies that $\operatorname{dev}\left(\gamma_{\lambda(m)}^{-1} \cdot w\right) \in S^{4 n+3}-S^{4 m-1}$. Thus $\operatorname{dev}\left(\gamma_{\lambda(m)}^{-1} \cdot w\right)$ is not contained in $S^{4 m-1}$, i.e., $\gamma_{\lambda(m)}^{-1} \cdot w \in \tilde{M}-\operatorname{dev}^{-1}\left(S^{4 m-1}\right)$. This shows that the Cauchy sequence $\left\{x_{i}\right\}$ converges in $\tilde{M}-\operatorname{dev}^{-1}\left(S^{4 m-1}\right)$ so that the simply connected space $\tilde{M}-\operatorname{dev}^{-1}\left(S^{4 m-1}\right)$ is complete. As a consequence, the local isometry dev : $\tilde{M}-\operatorname{dev}^{-1}\left(S^{4 m-1}\right) \longrightarrow S^{4 n+3}-S^{4 m-1}$ becomes a diffeomorphism. In particular, $\operatorname{dev}: \tilde{M} \longrightarrow \operatorname{dev}(\tilde{M})$ is a diffeomorphism. As $\Gamma$ is discrete and acts properly discontinuously on $\operatorname{dev}(\tilde{M}), \operatorname{dev}(\tilde{M}) \subset S^{4 n+3}-L(\Gamma)$. As above $L(\Gamma)=S^{4 m-1}$, so $\operatorname{dev}: \tilde{M} \longrightarrow S^{4 n+3}-S^{4 m-1}$ is diffeomorphic. Thus $M$ is isomorphic to $S^{4 n+3}-$ $S^{4 m-1} / \Gamma$ which is the double coset space $\mathrm{Sp}(m) \times \Delta \mathrm{Sp}(1) \times \mathrm{Sp}(n-m) \backslash \mathrm{Sp}(m, 1) \times$ $\operatorname{Sp}(n-m+1) / \Gamma$ where $2 \leqq m \leqq n$. This completes the proof of Theorem.

REMARK 31. (1) In the earlier draft of this paper, there was a gap in the completeness argument of the proof of Theorem 30, which was pointed out by the referee. Our proof (iii) to showing completeness is based on the observation of completeness argument of Suhyoung Choi and Lee's paper [10]. See also [21] for the correction.

(2) Notice that the transitive connected subgroup $H$ of $\operatorname{PSp}(n+1,1)$ is isomorphic to $\mathrm{Sp}(m, 1) \cdot \mathrm{Sp}(n-m+1)$ which acts transitively on $S^{4 n+3}-S^{4 m-1}$. Especially, $L(H)=S^{4 m-1}$. 
(3) When $m=1, \operatorname{PSp}(1,1)$ is isomorphic to the isometry group $\operatorname{PO}(4,1)^{0}$ of real hyperbolic space $\mathbb{H}_{\mathbb{R}}^{4}$. We do not know whether the above theorem is true or not in this case. The bending deformation as in Note 7 can not be applied to this case. B. Apanasov has noticed this fact by pointing out our incorrect use of bending for $\mathrm{PO}(4,1)^{0}=\operatorname{PSp}(1,1)$.

Proposition 32. Let $M$ be a compact spherical pseudo-quaternionic $(4 n+3)$ manifold $M$ and $G$ a connected semisimple Lie group with no compact factor and without center. Suppose that $\tilde{M}$ is not homogeneous. If the fundamental group $\pi$ is virtually isomorphic to a discrete uniform subgroup of $G$ and the cohomological dimension of $\pi$ is less than or equal to $4 n+3$, then $G$ must be isomorphic to one of the following Lie groups:

1. $\mathrm{PO}(m, 1)^{0}, 1 \leqq m \leqq 4 n+3$.

2. $\mathrm{PU}(m, 1), 1 \leqq m \leqq 2 n+1$.

Proof. Let $\Gamma$ be the holonomy group. As in the argument of Theorem 30, its real algebraic closure $\mathcal{A}(\Gamma)$ is either (i) $\operatorname{PSp}(n+1,1)$ or (ii) $\tau \circ \rho: \pi \rightarrow \tau(\Gamma)$ is isomorphic and discrete in $H$ where $H=\operatorname{PSp}(m, 1)(1 \leqq m \leqq n), \operatorname{PSL}(2, \mathbb{C}), \operatorname{PO}(n+1,1)$, or $\mathrm{PU}(n+1,1)$ and $\tau(\Gamma)$ is Zariski dense in $H$. Note that $\operatorname{PSL}(2, \mathbb{C}) \approx \mathrm{PO}(3,1)^{0}$.

Suppose that $G$ is isomorphic to neither $\mathrm{PO}(m, 1)^{0}$ nor $\mathrm{PU}(m, 1)$. Then $G$ has either $R$-rank $\geqq 2$ or is isomorphic to $\operatorname{PSp}(m, 1)(m \geqq 2)$ or $F_{4}^{-20}$. (Note that $\operatorname{PSp}(1,1) \approx \mathrm{PO}(4,1)^{0}$.) By the superrigidity, $\tau$ ○ $\rho$ extends to a continuous homomorphism $\varphi: G \longrightarrow \operatorname{PSp}(n+1,1)$ or $\varphi: G \longrightarrow H$. As $\varphi(G)$ is a connected Lie subgroup of $\operatorname{PSp}(n+1,1)$ or $H$, we have that $\varphi(G)=\operatorname{PSp}(n+1,1)$ or $\varphi(G)=H$. Put $\hat{G}=G / \operatorname{Ker} \varphi$ and let $p: G \rightarrow \hat{G}$ be the canonical projection.

Case (i) $\varphi$ induces an isomorphism $\hat{\varphi}: \hat{G} \longrightarrow \operatorname{PSp}(n+1,1)$. Since the cohomological dimension of $\pi$ is less than or equal to $4 n+3$ by the hypothesis, $\operatorname{dim} G / K \leqq$ $4 n+3$ where $K$ is a maximal compact subgroup. On the other hand, $\operatorname{dim} G / K \geqq$ $\operatorname{dim} \operatorname{PSp}(n+1,1) / \mathrm{Sp}(n+1) \cdot \operatorname{Sp}(1)=4 n+4$, which yields a contradiction.

Case (ii) $\hat{\varphi}: \hat{G} \longrightarrow H$ is an isomorphism. As $\tau \circ \rho: \pi \rightarrow \tau(\Gamma)$ is isomorphic, $p(\pi)$ maps isomorphically onto $\varphi(\pi)=\tau(\Gamma)$. Since $\tau(\Gamma)$ is discrete in $H, p(\pi)$ is discrete and cocompact in $\hat{G}$. If $K$ is a maximal compact subgroup of $G$, then $p(K)$ is a maximal compact subgroup of $\hat{G}$ by the hypothesis on $G$. There is a fibration of compact aspherical manifolds: $K \cap \operatorname{Ker} \varphi \backslash \operatorname{Ker} \varphi \rightarrow K \backslash G / \pi \stackrel{\hat{p}}{\longrightarrow} p(K) \backslash \hat{G} / p(\pi)$ in which $\hat{p}$ maps $\pi$ isomorphically onto $p(\pi)$. Since both compact aspherical manifolds have isomorphic fundamental groups and have the same dimension, $\hat{p}$ is a covering map and so is a homeomorphism. Hence the fiber $K \cap \operatorname{Ker} \varphi \backslash \operatorname{Ker} \varphi$ is a point or $K \cap \operatorname{Ker} \varphi=\operatorname{Ker} \varphi$ which is compact. As $G$ is semisimple with no normal compact factor and with no center, $\operatorname{Ker} \varphi$ must be trivial. Therefore $\varphi: G \longrightarrow H$ is an isomorphism, that is, $G=\operatorname{PSp}(m, 1)(m \geqq 2)$.

Suppose that $G$ is isomorphic to $\mathrm{PO}(m, 1)^{0}$, or $\mathrm{PU}(m, 1)$. The hypothesis that $\operatorname{dim} G / K \leqq 4 n+3$ implies that $1 \leqq m \leqq 4 n+3$ for $\mathrm{PO}(m, 1)^{0}$ and $1 \leqq m \leqq 2 n+1$ for $\mathrm{PU}(m, 1)$.

4.2. Deformation space on spherical Carnot-Carathéodory manifolds. Let $(\mathcal{G}, X)$ be a geometry and $M_{0}$ a $(\mathcal{G}, X)$-manifold. The deformation space $\mathcal{T}\left(M_{0}\right)$ is the space of all $(\mathcal{G}, X)$-structures on marked manifolds homeomorphic to $M_{0}$. More precisely, $\mathcal{T}\left(M_{0}\right)$ consists of equivalence classes of diffeomorphisms $f: M_{0} \rightarrow M$ from $M_{0}$ to $(\mathcal{G}, X)$-manifolds $M$. Two such diffeomorphisms $f_{i}: M_{0} \rightarrow M_{i}(i=1,2)$ are equivalent if and only if there is an isomorphism (i.e., $(\mathcal{G}, X)$-structure preserving 
diffeomorphism) $h: M_{1} \rightarrow M_{2}$ such that $h \circ f_{1}$ is isotopic to $f_{2}$. If $f: M_{0} \rightarrow M$ is a representative element of $\mathcal{T}\left(M_{0}\right)$, there is a developing pair $(\rho, \mathrm{dev}):\left(\pi_{1}(M), \tilde{M}\right) \rightarrow$ $(\mathcal{G}, X)$. We have the holonomy representation $\rho \circ f_{\sharp}: \pi_{1}\left(M_{0}\right) \rightarrow \mathcal{G}$ up to conjugacy. There is a map hol : $\mathcal{T}\left(M_{0}\right) \rightarrow \operatorname{Hom}(\Gamma, \mathcal{G}) / \mathcal{G}$ which assigns to a marked structure its holonomy representation. When we take $(\mathcal{G}, X)=\left(\operatorname{PSp}(n+1,1), S^{4 n+3}\right)$, the deformation space $\mathcal{T}\left(M_{0}\right)$ is the space of all possible spherical pseudo-quaternionic structures on $M_{0}$. Let $M_{0}=V_{-1}^{4 n+3} / \pi$ be a compact spherical pseudo-quaternionic $(4 n+3)$-manifold for $\pi \subset \mathrm{Sp}(n, 1) \cdot \mathrm{Sp}(1)$ as in (1.3). We have the following corollary from Theorem 30 .

COROllary 33. Let $M$ be a compact spherical pseudo-quaternionic $(4 n+3)$ manifold. Suppose that $\pi_{1}(M)$ is isomorphic to the fundamental group of a compact quaternionic hyperbolic $4 n$-manifold. Then, $M$ is pseudo-quaternionically isomorphic to $M_{0}=V_{-1}^{4 n+3} / \pi$.

Recall that a representation $\rho: \pi \rightarrow \operatorname{PSp}(n+1,1)$ is amenable if its closure of $\rho(\pi)$ lies in the maximal amenable Lie subgroup of $\operatorname{PSp}(n+1,1)$. A maximal amenable Lie group in $\operatorname{PSp}(n+1,1)$ is conjugate to $\operatorname{Sp}(n) \cdot \operatorname{Sp}(1)$ or $\operatorname{Sim}(\mathcal{M})$.

Let $\mathcal{S}(0, \infty)$ be the set of amenable representations in $\operatorname{Hom}(\pi, \operatorname{PSp}(n+1,1))$ and $\mathcal{S}(-1)$ the set of non-amenable representations in $\operatorname{Hom}(\pi, \operatorname{PSp}(n+1,1))$. Then the disjoint union $\mathcal{S}(0, \infty) \cup \mathcal{S}(-1)$ constitutes $\operatorname{Hom}(\pi, \operatorname{PSp}(n+1,1))$. A Fuchsian representation $\rho: \pi \rightarrow \operatorname{PSp}(n+1,1)$ is a discrete faithful representation whose image $\rho(\pi)$ leaves the totally geodesic quaternionic $n$-subspace $\mathbb{H}_{\mathbb{F}}^{n}$ in $\mathbb{H}_{\mathbb{F}}^{n+1}$ up to conjugacy (equivalently, leaves invariant the boundary sphere $S^{4 n-1}$ in $S^{4 n+3}$ ). (Compare [14], [8].)

LEMma 34. Let $\mathcal{R}$ (resp. $\mathcal{F}$ ) be the set of all discrete faithful (resp. Fuchsian) representations of $\pi$ into $\operatorname{PSp}(n+1,1)$. Then $\mathcal{S}(-1)=\mathcal{R}=\mathcal{F}$.

Proof. Let $\operatorname{Pr}: \operatorname{Sp}(n, 1) \cdot \operatorname{Sp}(1) \longrightarrow \operatorname{PSp}(n, 1)$ be the projection. $\operatorname{Put} \operatorname{Pr}(\pi)=\Gamma^{\prime}$. Since $\pi$ is isomorphic to a discrete uniform lattice $\Gamma^{\prime}$ in $\operatorname{PSp}(n, 1), \mathcal{R} \subset \mathcal{S}(-1)$. Let $\rho: \pi \longrightarrow \operatorname{PSp}(n+1,1)$ be a non-amenable representation. Put $\rho(\pi)=\Gamma$. Then the real algebraic closure $\mathcal{A}(\Gamma)$ is not amenable. As in the argument of the proof of Theorem 30, we have a homomorphism $\tau: \mathcal{A}(\Gamma) \longrightarrow H$, where $H$ is either $\mathrm{PSp}(m, 1), \mathrm{PU}(m, 1), \mathrm{PO}(m, 1),(2 \leqq m \leqq n+1)$ or $\operatorname{PSL}(2, \mathbb{C})$. Moreover, $\tau \circ \rho(\pi)$ is Zariski dense in $H$. Let $\tau \circ \rho \circ \mathrm{Pr}^{-1}: \Gamma^{\prime} \longrightarrow H$ be a homomorphism. By the superrigidity, there is an extension $\Psi: \operatorname{PSp}(n, 1) \rightarrow H$ of $\tau \circ \rho \circ \mathrm{Pr}^{-1}$. It is easy to see that $\Psi: \operatorname{PSp}(n, 1) \rightarrow H$ is an isomorphism. Then $\mathcal{A}(\Gamma)=\operatorname{Sp}(n, 1) \cdot \operatorname{Sp}(1)$ up to conjugacy. Since $\pi$ is torsion free, $\rho: \pi \rightarrow \Gamma \subset \operatorname{Sp}(n, 1) \cdot \operatorname{Sp}(1)$ is a discrete faithful representation. Moreover, $\operatorname{Sp}(n, 1) \cdot \operatorname{Sp}(1)$ acts transitively on $\mathbb{H}_{\mathbb{F}}^{n}$ in $\mathbb{H}_{\mathbb{F}}^{n+1}$, so $\rho$ is a Fuchsian representation. Hence $\mathcal{S}(-1)=\mathcal{R}$, which simultaneously coincides with $\mathcal{F}$.

Let $\operatorname{Pr}(\pi)=\Gamma^{\prime}$ be a discrete uniform lattice of $\operatorname{PSp}(n, 1)$ as above. If we put

$$
\mathcal{R}\left(\Gamma^{\prime}\right)=\left\{\rho^{\prime} \in \operatorname{Hom}\left(\Gamma^{\prime}, \operatorname{PSp}(n, 1)\right) \mid \rho^{\prime} \text { is a discrete faithful representation }\right\},
$$

then by the well known Mostow rigidity, the orbit space $\mathcal{R}\left(\Gamma^{\prime}\right) / \operatorname{PSp}(n, 1)$ is a single point.

Let $\mathcal{R}(\pi, \operatorname{Sp}(n, 1) \cdot \operatorname{Sp}(1))$ be the set of discrete faithful representations into $\operatorname{Sp}(n, 1) \cdot \operatorname{Sp}(1)$. Then it is easy to see that the set of discrete faithful representations $\mathcal{R}(\pi, \operatorname{PSp}(n+1,1)) / \operatorname{PSp}(n+1,1)$ is in one-to-one correspondence with 
$\mathcal{R}(\pi, \operatorname{Sp}(n, 1) \cdot \operatorname{Sp}(1)) / \operatorname{Sp}(n, 1) \cdot \operatorname{Sp}(1)$. (Compare [22].) As there is the fibration:

$$
\begin{aligned}
\operatorname{Hom}(\pi, \operatorname{Sp}(1)) / \operatorname{Sp}(1) & \longrightarrow \operatorname{Hom}(\pi, \operatorname{Sp}(n, 1) \cdot \operatorname{Sp}(1)) / \operatorname{Sp}(n, 1) \cdot \operatorname{Sp}(1) \\
& \longrightarrow \operatorname{Hom}\left(\Gamma^{\prime}, \operatorname{PSp}(n, 1)\right) / \operatorname{PSp}(n, 1),
\end{aligned}
$$

it follows that

$$
\operatorname{Hom}(\pi, \operatorname{Sp}(1)) / \operatorname{Sp}(1) \approx \mathcal{R}(\pi, \operatorname{Sp}(n, 1) \cdot \operatorname{Sp}(1)) / \operatorname{Sp}(n, 1) \cdot \operatorname{Sp}(1) .
$$

$\mathcal{R}=\mathcal{R}(\pi, \operatorname{PSp}(n+1,1)) \approx \operatorname{PSp}(n+1,1) \times \operatorname{Hom}(\pi, \operatorname{Sp}(1)) / \operatorname{Sp}(1)$. From Lemma 1.2 [17], note that $\mathcal{R}$ is a closed subset in $\operatorname{Hom}(\pi, \operatorname{PSp}(n+1,1))$.

COROLlaRY 35. The set of Fuchsian representations is a component of $\operatorname{Hom}(\pi, \operatorname{PSp}(n+1,1))$. Moreover, the set of Fuchsian representations is diffeomorphic to the space $\operatorname{PSp}(n+1,1) \times \operatorname{Hom}(\pi, \operatorname{Sp}(1)) / \operatorname{Sp}(1)$.

Proof. Since $\mathcal{S}(-1)=\mathcal{F} \approx \operatorname{PSp}(n+1,1) \times \operatorname{Hom}(\pi, \operatorname{Sp}(1)) / \operatorname{Sp}(1), \mathcal{S}(-1)$ is a closed connected subspace in $\operatorname{Hom}(\pi, \operatorname{PSp}(n+1,1))$. Let $\mathcal{C}(-1)$ be the connected component of $\operatorname{Hom}(\pi, \operatorname{PSp}(n+1,1))$ containing $\mathcal{S}(-1)$. If $\mathcal{C}(-1)-\mathcal{S}(-1) \neq \emptyset$, then there is a sequence $\left\{\rho_{i}\right\}$ such that $\lim _{i \rightarrow \infty} \rho_{i}=\rho \in \mathcal{S}(-1)$. Since $\operatorname{Hom}(\pi, \operatorname{PSp}(n+1,1))=$ $\mathcal{S}(0, \infty) \cup \mathcal{S}(-1)$, each $\rho_{i}$ is amenable. Suppose that each $\rho_{i}(\pi)$ stabilizes a point $\left\{\infty_{i}\right\}$. Passing to a subsequence, let $\lim _{i \rightarrow \infty} \infty_{i}=\infty \in S^{4 n+3}$. Then for every $\gamma \in \pi$,

$$
\rho(\gamma)(\infty)=\lim _{i \rightarrow \infty} \rho_{i}(\gamma)\left(\infty_{i}\right)=\lim _{i \rightarrow \infty} \infty_{i}=\infty
$$

which contradicts that $\rho$ is non-amenable.

Let hol $: \mathcal{T}\left(M_{0}\right) \rightarrow \operatorname{Hom}(\pi, \operatorname{PSp}(n+1,1)) / \operatorname{PSp}(n+1,1)$ be the map as before. We obtain the following from Corollary 33 and 35.

THEOREM 36. The map hol maps $\mathcal{T}\left(M_{0}\right)$ homeomorphically onto a connected component diffeomorphic to $\operatorname{Hom}(\pi, \operatorname{Sp}(1)) / \mathrm{Sp}(1)$.

\section{REFERENCES}

[1] D. A. Alekseevskir, Riemannian spaces with exceptional holonomy groups, Functional Anal. Appl., 2 (1968), pp. 97-105.

[2] Boris APANASOV AND XIANGDONG XIE, Geometrically finite complex hyperbolic manifolds, Internat. J. Math., 8 (1997), pp. 703-757.

[3] Boris Apanasov, Bending deformations of complex hyperbolic surfaces, Jour. fur die Reine und Angeandte Mathematik, 492 (1997), pp. 75-91.

[4] C. P. Boyer, K. Galicki, ANd B. M. ManN, 3-Sasakian manifolds, Proc. Japan Acad., 69 (1993), pp. 335-340.

[5] C. P. BOYER, K. GALICKI, AND B. M. MANN, The geometry and topology of 3-Sasakian manifolds, Jour. Reine and Ange. Math., 455 (1994), pp. 183-220.

[6] D.M. Burns AND S. SHNIDER, Spherical hypersurfaces in complex manifolds, Invent. Math., 33 (1976), pp. 223-246.

[7] J. Cheeger and D. G. EBIn, Comparison Theorems in Riemannian Geometry, Comp. Amsterdam-Oxford, 9, North-Holland Publ., 1975.

[8] S.S. Chen And L. Greenberg, Hyperbolic Spaces, in Contribution to Analysis, (A Collection of Papers Dedicated to Lipman Bers), L. Ahlfors, et. al. ed., Academic Press, New York and London, 1974, pp. 49-87.

[9] S.S. CHERN AND J. MOSER, Real hypersurfaces in complex manifolds, Acta Math., 133 (1974), pp. 48-69.

[10] Suhyoung ChoI AND HYUnKoo LEE, Geometric structure on manifolds and holonomyinvariant metrics, Forum Mathematicum., 9 (1997), pp. 247-256. 
[11] K. CoRLETTE, Archimedean superrigidity and hyperbolic geometry, Ann. of Math., 135 (1992), pp. 165-182.

[12] D. FrIED, Closed similarity manifolds, Comment. Math. Helv., 55 (1980), pp. 576-582.

[13] W. Goldman, Complex Hyperbolic Geometry, Oxford Mathematical Monographs, Oxford Univ. Press, 1999.

[14] W. GOLDMAN AND J. MILLSON, Local rigidity of discrete subgroups acting on complex hyperbolic space, Invent. Math., 88 (1987), pp. 495-520.

[15] S. Helgason, Differential Geometry and Symmetric Spaces, Academic Press, New York and London, 1962.

[16] S. IsHiHARA, Quaternion Kählerian manifolds, J. Diff. Geom., 9 (1974), pp. 483-500.

[17] D. JoHNSON AND J. MILlson, Deformation spaces associated to compact hyperbolic manifolds, in Discrete Groups in Geometry and Analysis (Proceedings of a conference held at Yale University in honor of G.D. Mostow), Progress in Math., Boston, Birkhäuser, 1987, pp. 48-106

[18] Y. KAMISHIMA, Conformally flat manifolds whose development maps are not surjective. I, Trans. Amer. Math. Soc., 294 (1986), pp. 607-623.

[19] Y. KAMISHIMA, Geometric flows on compact manifolds and Global rigidity, Topology, 35 (1996), pp. 439-450.

[20] Y. KAMISHIMA, Standard pseudo-Hermitian structure and Seifert fibration on $C R$ manifold, Annals of Global Analysis and Geometry, 12 (1994), pp. 261-289.

[21] Y. KaMishima, Correction to developing maps in geometric structures, Preprint.

[22] Y. Kamishima AND S. T. TAN, Deformation spaces on geometric structures, in Aspects of Low dimensional Manifolds, Y. Matsumoto and S. Morita, ed., Advanced studies in pure math., 20 (1992), pp. 263-299.

[23] Y. Kamishima AND T. TsuboI, CR-structures on Seifert manifolds, Invent. Math., 104 (1991), pp. 149-163.

[24] S. KoBAYASHI, Transformation Groups in Differential Geometry, Ergebnisse Math., 70, Springer-Verlag, 1970.

[25] S. KoBAYASHI AND K. NomIzU, Foundations of Differential Geometry I, II, Interscience John Wiley \& Sons, New York, 1969.

[26] M. KONISHI, On manifolds with Sasakian 3-structure over quaternion Kaehler manifolds, K odai Math. Sem. Rep., 26 (1975), pp. 194-200.

[27] J, L. KoszuL, Lectures on Groups of Transformations, Tata Institute of Fundamental Research, Bombay, 1965.

[28] R. KULKaRNI, On the principle of uniformization, J. Diff. Geom., 13 (1978), pp. 109-138.

[29] R. KUlKarni, Proper actions and pseudo-Riemannian space forms, Adv. Math., 40 (1981), pp. 10-51.

[30] J. LeLONG-FERRAND, Transformations conformes et quasiconformes des variétés riemanniennes compactes, Acad. Roy. Belgique Sci. Mem. Coll., 8 (1971), pp. 1-44.

[31] R. MINER, Spherical C.R-manifolds with amenable holonomy, International J. of Math., 1:4 (1991), pp. 479-501.

[32] J. Mitchell, On Carnot-Carathéodory metrics, J. Diff. Geom., 21 (1985), pp. 109-138.

[33] N. MoK, Y-T. SIU, ANI S-K.YeUNG, Geometric superrigidity, Invent. Math., 113 (1993), pp. $57-83$.

[34] G. A. MARGULIS AND G. D. MOSTOW, The differentiai of a quasi-conformal mapping of a Carnot-Carathéodory space, Geom. and Func. Analysis, 5 (1995), pp. 402-433.

[35] M. ОватA, The conjectures on conformal transformations of Riemannian manifolds, J. Diff. Geom., 6 (1971), pp. 247-258.

[36] T. OchIAI, Geometry associated with semisimple flat homogeneous spaces, Trans. Amer. Math. Soc., 152 (1970), pp. 159-193.

[37] M. Raghunathan, Discrete Subgroups of Lie Groups, Ergebnisse Math. u.i. Grenzgeb., 68, Springer, Berlin, New York, 1972.

[38] S. SAlamon, Riemannian Geometry and Holonomy Groups, Pitman research notes in Math., 201, Longman Scientific, 1989.

[39] S. Salamon, Differential geometry of quaternionic manifolds, Ann. Scient. Éc. Norm. Sup., IV., 19 (1986), pp. 31-55.

[40] S. SASAKI, Spherical space forms with normal contact metric 3-structure, J. Diff. Geom., 6 (1972), pp. 307-315.

[41] S. TANNO, Killing vector fields on contact Riemannian manifolds and fibering related to the Hopf fibrations, Tôhoku Math. Jour., 23 (1971), pp. 313-333.

[42] W. Thurston, The Geometry and Topology of 3-Manifolds, Lecture Notes, The Geometry Center, Minnesota, 1992. 
[43] S. WeBster, Pseudo-Hermitian structures on a real hypersurface, J. Diff. Geom., 13 (1978), pp. 25-41.

[44] S. WEBSTER, On the pseudo conformal geometry of a Kähler manifold, Jour. Math. Z., 157 (1977), pp. 265-270.

[45] S. WEBSTER, On the transformation group of a real hypersurface, Trans. Amer. Math. Soc., 231 (1977), pp. 179-190.

[46] J. Wolf, Spaces of Constant Curvature, McGraw-Hill, Inc., 1967.

[47] R. ZIMMER, Ergodic Theory and Semisimple Groups, Monographs in Mathematics, Boston, Birkhäuser, 1985. 
